\title{
ENTROPIC PROJECTIONS AND DOMINATING POINTS
}

\author{
Christian LÉONARD ${ }^{1}$
}

\begin{abstract}
Entropic projections and dominating points are solutions to convex minimization problems related to conditional laws of large numbers. They appear in many areas of applied mathematics such as statistical physics, information theory, mathematical statistics, ill-posed inverse problems or large deviation theory. By means of convex conjugate duality and functional analysis, criteria are derived for the existence of entropic projections, generalized entropic projections and dominating points. Representations of the generalized entropic projections are obtained. It is shown that they are the "measure component" of the solutions to some extended entropy minimization problem. This approach leads to new results and offers a unifying point of view. It also permits to extend previous results on the subject by removing unnecessary topological restrictions. As a by-product, new proofs of already known results are provided.
\end{abstract}

Mathematics Subject Classification. 60F10,60F99, 60G57, 46N10.

Received January 11, 2008. Revised January 12, 2009.

\section{INTRODUCTION}

Entropic projections and dominating points are solutions to convex minimization problems related to conditional laws of large numbers. They appear in many areas of applied mathematics such as statistical physics, information theory, mathematical statistics, ill-posed inverse problems or large deviation theory.

\subsection{Conditional laws of large numbers}

Suppose that the empirical measures

$$
L_{n}:=\frac{1}{n} \sum_{i=1}^{n} \delta_{Z_{i}}, \quad n \geq 1
$$

of the $\mathcal{Z}$-valued random variables $Z_{1}, Z_{2}, \ldots\left(\delta_{z}\right.$ is the Dirac measure at $\left.z\right)$ obey a Large Deviation Principle (LDP) in the set $P_{\mathcal{Z}}$ of all probability measures on $\mathcal{Z}$ with the rate function $I$. This approximately means that $\mathbb{P}\left(L_{n} \in \mathcal{A}\right) \underset{n}{\asymp} \exp \left[-n \inf _{P \in \mathcal{A}} I(P)\right]$ for $\mathcal{A} \subset P_{\mathcal{Z}}$. With regular enough subsets $\mathcal{A}$ and $\mathcal{C}$ of $P_{\mathcal{Z}}$, we can expect that for "all" $\mathcal{A}$

$$
\lim _{n \rightarrow \infty} \mathbb{P}\left(L_{n} \in \mathcal{A} \mid L_{n} \in \mathcal{C}\right)= \begin{cases}1, & \text { if } \mathcal{A} \ni P_{*} \\ 0, & \text { otherwise }\end{cases}
$$

Keywords and phrases. Conditional laws of large numbers, random measures, large deviations, entropy, convex optimization, entropic projections, dominating points, Orlicz spaces.

${ }^{1}$ Modal-X, Université Paris Ouest, Bât. G, 200 av. de la République, 92000 Nanterre, France; leonard@u-paris10.fr 
where $P_{*}$ is a minimizer of $I$ on $\mathcal{C}$. To see this, remark that (formally) $\mathbb{P}\left(L_{n} \in \mathcal{A} \mid L_{n} \in \mathcal{C}\right) \underset{n \rightarrow \infty}{\asymp} \exp \left[-n\left(\inf _{P \in \mathcal{A} \cap \mathcal{C}}\right.\right.$ $\left.\left.I(P)-\inf _{P \in \mathcal{C}} I(P)\right)\right]$. A rigorous statement is given at Theorem 7.1.

If $\left(Z_{1}, \ldots, Z_{n}\right)$ is exchangeable for each $n$, then (1.2) is equivalent to

$$
\mathcal{L}\left(Z_{1} \mid L_{n} \in \mathcal{C}\right) \underset{n \rightarrow \infty}{\longrightarrow} P_{*}
$$

which means that conditionally on $L_{n} \in \mathcal{C}$, the law of any tagged "particle" (here we chose the first one) tends to $P_{*}$ as $n$ tends to infinity.

If $I$ is strictly convex and $\mathcal{C}$ is convex, $P_{*}$ is unique and (1.2) roughly means that conditionally on $L_{n} \in \mathcal{C}$, as $n$ tends to infinity $L_{n}$ tends to the solution $P_{*}$ of the minimization problem

$$
\text { minimize } I(P) \text { subject to } P \in \mathcal{C}, \quad P \in P_{\mathcal{Z}} \text {. }
$$

Such conditional Laws of Large Numbers (LLN) appear in information theory and in statistical physics where they are often called Gibbs conditioning principles (see [9], Sect. 7.3 and the references therein). If the variables $Z_{i}$ are independent and identically distributed with law $R$, the LDP for the empirical measures is given by Sanov's theorem and the rate function $I$ is the relative entropy

$$
I(P \mid R)=\int_{\mathcal{Z}} \log \left(\frac{\mathrm{d} P}{\mathrm{~d} R}\right) \mathrm{d} P, \quad P \in P_{\mathcal{Z}}
$$

Instead of the empirical probability measure of a random sample, one can consider another kind of random measure. Let $z_{1}, z_{2}, \ldots$ be deterministic points in $\mathcal{Z}$ such that the empirical measure $\frac{1}{n} \sum_{i=1}^{n} \delta_{z_{i}}$ converges to $R \in P_{\mathcal{Z}}$. Let $W_{1}, W_{2}, \ldots$ be a sequence of independent random real variables. The random measure of interest is

$$
L_{n}=\frac{1}{n} \sum_{i=1}^{n} W_{i} \delta_{z_{i}}
$$

where the $W_{i}$ 's are interpreted as random weights. If the weights are independent copies of $W$, as $n$ tends to infinity, $L_{n}$ tends to the deterministic measure $\mathbb{E} W . R$ and obeys the LDP in the space $M_{\mathcal{Z}}$ of measures on $\mathcal{Z}$ with rate function $I(Q)=\int_{\mathcal{Z}} \gamma^{*}\left(\frac{\mathrm{d} Q}{\mathrm{~d} R}\right) \mathrm{d} R, Q \in M_{\mathcal{Z}}$ where $\gamma^{*}$ is the Cramér transform of the law of $W$. In case the $W_{i}$ 's are not identically distributed, but have a law which depends (continuously) on $z_{i}$, one can again show that under additional assumptions $L_{n}$ obeys the LDP in $M_{\mathcal{Z}}$ with rate function

$$
I(Q)=\left\{\begin{array}{ll}
\int_{\mathcal{Z}} \gamma_{z}^{*}\left(\frac{\mathrm{d} Q}{\mathrm{~d} R}(z)\right) R(\mathrm{~d} z), & \text { if } Q \prec R \\
+\infty, & \text { otherwise, }
\end{array} \quad Q \in M_{\mathcal{Z}}\right.
$$

where $\gamma_{z}^{*}$ is the Cramér transform of $W_{z}$. As $\gamma^{*}$ is the convex conjugate of the log-Laplace transform of $W$, it is a convex function: $I$ is a convex integral functional which is often called an entropy. Again, conditional LLNs hold for $L_{n}$ and lead to the entropy minimization problem:

$$
\text { minimize } I(Q) \text { subject to } Q \in \mathcal{C}, \quad Q \in M_{\mathcal{Z}} \text {. }
$$

The large deviations of these random measures and their conditional LLNs enter the framework of Maximum Entropy in the Mean (MEM) which has been studied among others by Csiszár et al. [7], Dacunha-Castelle and Gamboa [8], Gamboa and Gassiat [12] and Najim [21] and also [9] (Thm. 7.2.3). This problem also arises in the context of statistical physics. It has been studied among others by Boucher et al. [3] and Ellis et al. [11].

The relative entropy corresponds to $\gamma^{*}(t)=t \log t-t+1$ in (1.5): the problem (1.3) is a special case of (1.6) with the additional constraint that $Q(\mathcal{Z})=1$. 
In this paper, the constraint set $\mathcal{C}$ is assumed to be convex as is almost always done in the literature on the subject. This allows to rely on convex analysis, saddle-point theory or on the geometric theory of projection on convex sets.

The convex indicator of a subset $A$ of $X$ is denoted by

$$
\iota_{A}(x)=\left\{\begin{array}{ll}
0, & \text { if } x \in A \\
+\infty, & \text { otherwise, }
\end{array} \quad x \in X .\right.
$$

Note that although $\iota_{A}$ is a convex function if and only if $A$ is a convex set, we call it a convex indicator in any case to differentiate it from the probabilistic indicator $\mathbf{1}_{A}$.

\subsection{Examples}

We give a short list of popular entropies. They are described at (1.5) and characterized by their integrand $\gamma^{*}$.

(1) The relative entropy: $\gamma^{*}(t)=t \log t-t+1+\iota_{\{t \geq 0\}}$.

(2) The reverse relative entropy: $\gamma^{*}(t)=t-\log t-1+\iota_{\{t>0\}}$.

(3) The Fermi-Dirac entropy: $\gamma^{*}(t)=\frac{1}{2}[(1+t) \log (1+t)+(1-t) \log (1-t)]+\iota_{\{-1 \leq t \leq 1\}}$.

(4) The $L_{p}$ norm $(1<p<\infty): \gamma^{*}(t)=|t|^{p} / p$ and

(5) the $L_{p}$ entropy $(1<p<\infty): \gamma^{*}(t)=t^{p} / p+\iota_{\{t \geq 0\}}$.

Note that the reverse relative entropy is $P \mapsto I(R \mid P)$.

In the case of the relative and reverse relative entropies, the global minimizer is $R$ and one can interpret $I(P \mid R)$ and $I(R \mid P)$ as some type of distances between $P$ and $R$. Note also that the positivity of the minimizers of (1.6) is guaranteed by $\left\{\gamma^{*}<\infty\right\} \subset[0, \infty)$. Consequently, the restriction that $P$ is a probability measure is insured by the only unit mass constraint $P(\mathcal{Z})=1$.

A very interesting Bayesian interpretation of (1.6) is obtained in [8,12] in terms of the LDPs for $L_{n}$ defined at (1.4). The above entropies correspond to iid weights $W$ distributed as follows:

(1) the relative entropy: $\operatorname{Law}(W)=\operatorname{Poisson}(1)$,

(2) the reverse relative entropy: $\operatorname{Law}(W)=\operatorname{Exponential}(1)$,

(3) the Fermi-Dirac entropy: $\operatorname{Law}(W)=\left(\delta_{-1}+\delta_{+1}\right) / 2$,

(4) the $L_{2}$ norm: $\operatorname{Law}(W)=\operatorname{Normal}(0,1)$.

\subsection{Entropic and generalized entropic projections}

The minimizers of (1.6) are called entropic projections. It may happen that even if the minimizer is not attained, any minimizing sequence converges to some measure $Q_{*}$ which does not belong to $\mathcal{C}$. This intriguing phenomenon was discovered by Csiszár [5]. Such a $Q_{*}$ is called a generalized entropic projection.

In the special case where $I$ is the relative entropy, Csiszár has obtained existence results in [4] together with dual equalities. His proofs are based on geometric properties of the relative entropy; no convex analysis is needed. Based on the same geometric ideas, he obtained later in [5] a powerful Gibbs conditioning principle for noninteracting particles. For general entropies as in (1.5), he studies the problem of existence of entropic and generalized entropic projections in [6].

The minimization problem (1.6) is interesting in its own right, even when conditional LLNs are not at stake. The literature on this subject is huge, for instance see [13,29] and the references therein.

\subsection{The extended minimization problem}

As will be seen, it is worth introducing an extension of (1.6) to take the generalized projections into account. A solution to (1.6) is in $A_{\mathcal{Z}}$ : the space of all $Q \in M_{\mathcal{Z}}$ which are absolutely continuous with respect to $R$. We consider an extension $\bar{I}$ of the entropy $I$ to a vector space $L_{\mathcal{Z}}$ which is the direct sum $L_{\mathcal{Z}}=A_{\mathcal{Z}} \oplus S_{\mathcal{Z}}$ of $A_{\mathcal{Z}}$ and a vector space $S_{\mathcal{Z}}$ of singular linear forms (acting on numerical functions) which may not be $\sigma$-additive. 
Any $\ell$ in $L_{\mathcal{Z}}$ is uniquely decomposed into $\ell=\ell^{a}+\ell^{s}$ with $\ell^{a} \in A_{\mathcal{Z}}$ and $\ell^{s} \in S_{\mathcal{Z}}$ and $\bar{I}$ has the following shape

$$
\bar{I}(\ell)=I\left(\ell^{a}\right)+I^{s}\left(\ell^{s}\right)
$$

where $I^{s}$ is a positively homogeneous function on $S_{\mathcal{Z}}$. See (2.9) for the precise description of $\bar{I}$. For instance, the extended relative entropy is

$$
\bar{I}(\ell \mid R)=I\left(\ell^{a} \mid R\right)+\sup \left\{\left\langle\ell^{s}, u\right\rangle ; u, \int_{\mathcal{Z}} \mathrm{e}^{u} \mathrm{~d} R<\infty\right\}, \quad \ell \in L_{\mathcal{Z}}
$$

and actually $\left\langle\ell^{s}, u\right\rangle=0$ for any $\ell$ such that $\bar{I}(\ell)<\infty$ and any $u$ such that $\int_{\mathcal{Z}} \mathrm{e}^{a|u|} \mathrm{d} R<\infty$ for all $a>0$. The reverse entropy, $L_{1}$-norm and $L_{1}$-entropy also admit nontrivial extensions. On the other hand, the extensions of the Fermi-Dirac, $L_{p}$-norm and $L_{p^{-}}$entropy with $p>1$ are trivial: $\left\{k \in S_{\mathcal{Z}} ; I^{s}(k)<\infty\right\}=\{0\}$.

The extended problem is

$$
\text { minimize } \bar{I}(\ell) \text { subject to } \ell \in \mathcal{C}, \quad \ell \in L_{\mathcal{Z}} \text {. }
$$

In fact, $\bar{I}$ is chosen to be the largest convex lower semicontinuous extension of $I$ to $L_{\mathcal{Z}}$ with respect to some weak topology. This guarantees tight relations between (1.6) and (1.8). In particular, one can expect that their values are equal for a large class of convex sets $\mathcal{C}$.

Even if $I$ is strictly convex, $\bar{I}$ isn't strictly convex in general since $I^{s}$ is positively homogeneous, so that (1.8) may admit several minimizers.

Examples will be given of interesting situations where (1.6) is not attained in $A_{\mathcal{Z}}$ while (1.8) is attained in $L_{\mathcal{Z}}$.

\subsection{Dominating points}

Let the constraint set $\mathcal{C}$ be described by

$$
\mathcal{C}=\left\{Q \in M_{\mathcal{Z}} ; T Q \in C\right\}
$$

where $C$ is a subset of a vector space $\mathcal{X}$ and $T: M_{\mathcal{Z}} \rightarrow \mathcal{X}$ is a linear operator. As a typical example, one can think of

$$
T Q=\int_{\mathcal{Z}} \theta(z) Q(\mathrm{~d} z)
$$

where $\theta: \mathcal{Z} \rightarrow \mathcal{X}$ is some function and the integral should be taken formally for the moment. With $L_{n}$ given at (1.1) or (1.4), if $T$ is regular enough, we obtain by the contraction principle that $X_{n}:=T L_{n}=\frac{1}{n} \sum_{i=1}^{n} \theta\left(Z_{i}\right) \in$ $\mathcal{X}$ or $X_{n}:=T L_{n}=\frac{1}{n} \sum_{i=1}^{n} W_{i} \theta\left(z_{i}\right)$ obeys the LDP in $\mathcal{X}$ with rate function $J(x)=\inf \left\{I(Q) ; Q \in M_{\mathcal{Z}}, T Q=x\right\}$, $x \in \mathcal{X}$. Once again, the conditional LLN for $X_{n}$ is of the form: For "all" $A \subset \mathcal{X}$,

$$
\lim _{n \rightarrow \infty} \mathbb{P}\left(X_{n} \in A \mid X_{n} \in C\right)= \begin{cases}1, & \text { if } A \ni x_{*} \\ 0, & \text { otherwise }\end{cases}
$$

where $x_{*}$ is a solution to the minimization problem

$$
\text { minimize } J(x) \text { subject to } x \in C, \quad x \in \mathcal{X} \text {. }
$$

The minimizers of (1.11) are called dominating points. This notion was introduced by Ney [22,23] in the special case where $\left(Z_{i}\right)_{i \geq 1}$ is an iid sequence in $\mathcal{Z}=\mathbb{R}^{d}$ and $\theta$ is the identity, i.e. $X_{n}=\frac{1}{n} \sum_{i=1}^{n} Z_{i}$. Later, Einmahl and Kuelbs $[10,14]$ have extended this study to a Banach space $\mathcal{Z}$. In this iid case, $J$ is the Cramér transform of the law of $Z_{1}$. 


\subsection{Presentation of the results}

We treat the problems of existence of entropic projections and dominating points in a unified way, taking advantage of the mapping $T Q=x$. Hence, we mainly concentrate on the entropic projections and then transport the results to the dominating points.

It will be proved at Proposition 5.4 that the entropic projection exists on $\mathcal{C}$ if the supporting hyperplanes of $\mathcal{C}$ are directed by sufficiently integrable functions. In some cases of not enough integrable supporting hyperplanes, the representation of the generalized projection is still available and given at Theorem 5.6.

It will appear that the generalized projection is the "measure" part of the minimizers of (1.8). For instance, with the relative entropy $I(. \mid R)$, the projection exists in $\mathcal{C}$ if its supporting hyperplanes are directed by functions $u$ such that $\int_{\mathcal{Z}} \mathrm{e}^{\alpha|u|} \mathrm{d} R<\infty$ for all $\alpha>0$, see Proposition 5.7. If these $u$ only satisfy $\int_{\mathcal{Z}} \mathrm{e}^{\alpha|u|} \mathrm{d} R<\infty$ for some $\alpha>0$, the projection may not exist in $\mathcal{C}$, but the generalized projection is computable: its Radon-Nykodym derivative with respect to $R$ is characterized at Proposition 5.8.

We find again some already known results of Csiszár [5,6], Einmahl and Kuelbs [10,14] with different proofs and a new point of view. The representations of the generalized projections are new results. The conditions on $C$ to obtain dominating points are improved and an interesting phenomenon noticed in [14] is clarified at Remark 6.8 by connecting it with the generalized entropic projection.

Finally, a probabilistic interpretation of the singular components of the generalized projections is proposed at Section 7. It is obtained in terms of conditional LLNs.

The main results are Theorems 4.1, 4.4, 5.6 and 6.7.

\subsection{Outline of the paper}

At Section 2, we give precise formulations of the entropy minimization problems (1.6) and (1.8). Then we recall at Theorems 2.7 and 2.8 results from [19] about the existence and uniqueness of the solutions of these problems, related dual equalities and the characterizations of their solutions in terms of integral representations.

Examples of standard entropies and constraints are presented at Section 3.

We show at Theorem 4.4 in Section 4 that under "critical" constraints, although the problem (1.6) may not be attained, its minimizing sequences may converge in some sense to some measure $Q_{*}$ : the generalized entropic projection.

Section 5 is mainly a restatement of Sections 2 and 4 in terms of entropic projections. The results are also stated explicitly for the special important case of the relative entropy.

Section 6 is devoted to dominating points. As they are continuous images of entropic projections, the main results of this section are corollaries of the results of Section 5 .

At Section 7, we illustrate our results in terms of conditional LLNs, see (1.2). In particular, the generalized projections and singular components of the minimizers of (1.8) are interpreted in terms of these conditional LLNs.

\subsection{Notation}

Let $X$ and $Y$ be topological vector spaces. The algebraic dual space of $X$ is $X^{*}$, the topological dual space of $X$ is $X^{\prime}$. The topology of $X$ weakened by $Y$ is $\sigma(X, Y)$ and we write $\langle X, Y\rangle$ to specify that $X$ and $Y$ are in separating duality.

Let $f: X \rightarrow[-\infty,+\infty]$ be an extended numerical function. Its convex conjugate with respect to $\langle X, Y\rangle$ is

$$
f^{*}(y)=\sup _{x \in X}\{\langle x, y\rangle-f(x)\} \in[-\infty,+\infty], \quad y \in Y
$$

Its subdifferential at $x$ with respect to $\langle X, Y\rangle$ is

$$
\partial_{Y} f(x)=\{y \in Y ; f(x+\xi) \geq f(x)+\langle y, \xi\rangle, \forall \xi \in X\} .
$$

If no confusion occurs, we write $\partial f(x)$. 
Let $A$ be a subset of $X$, its intrinsic core is icor $A=\left\{x \in A ; \forall x^{\prime} \in \operatorname{aff} A, \exists t>0,\left[x, x+t\left(x^{\prime}-x\right)[\subset A\}\right.\right.$ where aff $A$ is the affine space spanned by $A$. Let us denote $\operatorname{dom} f=\{x \in X ; f(x)<\infty\}$ the effective domain of $f$ and icordom $f$ the intrinsic core of $\operatorname{dom} f$.

The convex indicator $\iota_{A}$ of a subset $A$ of $X$ is defined at (1.7).

We write

and $I=I_{\gamma^{*}}$ for short, instead of (1.5).

$$
I_{\varphi}(u):=\int_{\mathcal{Z}} \varphi(z, u(z)) R(\mathrm{~d} z)=\int_{\mathcal{Z}} \varphi(u) \mathrm{d} R
$$

The inf-convolution of $f$ and $g$ is $f \square g(z)=\inf \{f(x)+g(y) ; x, y: x+y=z\}$.

An index of notation is provided at the end of the article.

\section{Minimizing Entropy Under CONVEX CONSTRAints}

In this section, the main results of [19] are recalled. They are Theorems 2.7 and 2.8 below and their statements necessitate the notion of Orlicz spaces. First, we recall basic definitions and notions about these function spaces which are natural extensions of the standard $L_{p}$ spaces.

\subsection{Orlicz spaces}

The fact that the generalized projection may not belong to $\mathcal{C}$ is connected with some properties of Orlicz spaces associated to $I$. Let us recall some basic definitions and results. A standard reference is [24].

A set $\mathcal{Z}$ is furnished with a $\sigma$-finite nonnegative measure $R$ on a $\sigma$-field which is assumed to be $R$-complete. A function $\rho: \mathcal{Z} \times \mathbb{R}$ is said to be a Young function if for $R$-almost every $z, \rho(z, \cdot)$ is a convex even $[0, \infty]$ valued function on $\mathbb{R}$ such that $\rho(z, 0)=0$ and there exists a measurable function $z \mapsto s_{z}>0$ such that $0<\rho\left(z, s_{z}\right)<\infty$.

In the sequel, every numerical function on $\mathcal{Z}$ is supposed to be measurable.

Definition 2.1 (the Orlicz spaces $L_{\rho}$ and $E_{\rho}$ ). The Orlicz space associated with $\rho$ is defined by $L_{\rho}=\{u: \mathcal{Z} \rightarrow$ $\left.\mathbb{R} ;\|u\|_{\rho}<+\infty\right\}$ where the Luxemburg norm $\|\cdot\|_{\rho}$ is defined by

$$
\|u\|_{\rho}=\inf \left\{\beta>0 ; \int_{\mathcal{Z}} \rho(z, u(z) / \beta) R(\mathrm{~d} z) \leq 1\right\}
$$

and $R$-a.e. equal functions are identified. Hence,

$$
L_{\rho}=\left\{u: \mathcal{Z} \rightarrow \mathbb{R} ; \exists \alpha_{o}>0, \int_{\mathcal{Z}} \rho\left(z, \alpha_{o} u(z)\right) R(\mathrm{~d} z)<\infty\right\}
$$

A subspace of interest is

$$
E_{\rho}=\left\{u: \mathcal{Z} \rightarrow \mathbb{R} ; \forall \alpha>0, \int_{\mathcal{Z}} \rho(z, \alpha u(z)) R(\mathrm{~d} z)<\infty\right\} .
$$

Taking $\rho_{p}(z, s)=|s|^{p} / p$ with $p \geq 1$ gives $L_{\rho_{p}}=E_{\rho_{p}}=L_{p}$ and the corresponding Luxemburg norm is $\|\cdot\|_{\rho_{p}}=$ $p^{-1 / p}\|\cdot\|_{p}$ where $\|\cdot\|_{p}$ is the usual $L_{p}$ norm.

The convex conjugate $\rho^{*}$ of a Young function is still a Young function, so that $L_{\rho^{*}}$ is also an Orlicz space. Hölder's inequality in Orlicz spaces is

$$
\|u v\|_{1} \leq 2\|u\|_{\rho}\|v\|_{\rho^{*}}, \quad u \in L_{\rho}, v \in L_{\rho^{*}} .
$$

For instance, with $\rho_{p}^{*}(t)=|t|^{q} / q, 1 / p+1 / q=1,(2.1)$ is $\|u v\|_{1} \leq 2 p^{-1 / p} q^{-1 / q}\|u\|_{p}\|v\|_{q}$. Note that $2 p^{-1 / p} q^{-1 / q} \geq$ 1 with equality when $p=2$. 
Theorem 2.2 (representation of $\left.E_{\rho}^{\prime}\right)$. Suppose that $\rho$ is a finite Young function. Then, the dual space of $E_{\rho}$ is isomorphic to $L_{\rho^{*}}$, the Orlicz space associated with the Young function $\rho^{*}$ which is the convex conjugate of $\rho$.

A continuous linear form $\ell \in L_{\rho}^{\prime}$ is said to be singular if for all $u \in L_{\rho}$, there exists a decreasing sequence of measurable sets $\left(A_{n}\right)$ such that $R\left(\cap_{n} A_{n}\right)=0$ and for all $n \geq 1,\left\langle\ell, u \mathbf{1}_{\mathcal{Z} \backslash A_{n}}\right\rangle=0$.

Proposition 2.3. Let us assume that $\rho$ is finite. Then, $\ell \in L_{\rho}^{\prime}$ is singular if and only if $\langle\ell, u\rangle=0$, for all $u$ in $E_{\rho}$.

Let us denote respectively $L_{\rho^{*}} R=\left\{f R ; f \in L_{\rho^{*}}\right\}$ and $L_{\rho}^{s}$ the subspaces of $L_{\rho}^{\prime}$ of all absolutely continuous and singular forms.

Theorem 2.4 (representation of $L_{\rho}^{\prime}$ ). Let $\rho$ be any Young function. The dual space of $L_{\rho}$ is isomorphic to the direct sum $L_{\rho}^{\prime}=L_{\rho^{*}} R \oplus L_{\rho}^{s}$. This implies that any $\ell \in L_{\rho}^{\prime}$ is uniquely decomposed as

$$
\ell=\ell^{a}+\ell^{s}
$$

with $\ell^{a} \in L_{\rho^{*}} R$ and $\ell^{s} \in L_{\rho}^{s}$.

In the decomposition (2.2), $\ell^{a}$ is called the absolutely continuous part of $\ell$ while $\ell^{s}$ is its singular part.

The function $\rho$ is said to satisfy the $\Delta_{2}$-condition if

$$
\text { there exist } \kappa>0, s_{o} \geq 0 \text { such that } \forall s \geq s_{o}, z \in \mathcal{Z}, \rho_{z}(2 s) \leq \kappa \rho_{z}(s) \text {. }
$$

When $R$ is bounded, in order that $E_{\rho}=L_{\rho}$, it is enough that $\rho$ satisfies the $\Delta_{2}$-condition. Consequently, in this situation we have $L_{\rho}^{\prime}=L_{\rho^{*}} R$ so that $L_{\rho}^{s}$ reduces to the null vector space.

We shall see below that the important case of the relative entropy leads to a Young function $\rho$ which doesn't satisfy the $\Delta_{2}$-condition; non-trivial singular components will appear.

\subsection{The assumptions}

Let $R$ be a positive measure on a space $\mathcal{Z}$ and take a $[0, \infty]$-valued measurable function $\gamma^{*}$ on $\mathcal{Z} \times \mathbb{R}$ to define $I$ as at (1.5). In order to define the constraint in a way similar to (1.10), take a function

$$
\theta: \mathcal{Z} \rightarrow \mathcal{X}_{o}
$$

where $\mathcal{X}_{o}$ is the algebraic dual space of some vector space $\mathcal{Y}_{o}$. Let us collect the assumptions on $R, \gamma^{*}, \theta$ and $C$.

\subsection{Assumptions (A).}

$\left(\mathrm{A}_{R}\right)$ It is assumed that the reference measure $R$ is a bounded positive measure on a space $\mathcal{Z}$ endowed with some $R$-complete $\sigma$-field.

$\left(\mathrm{A}_{C}\right) C$ is a convex subset of $\mathcal{X}_{o}$.

$\left(\mathrm{A}_{\gamma^{*}}\right)$ Assumptions on $\gamma^{*}$.

(1) $\gamma^{*}(\cdot, t)$ is $z$-measurable for all $t$ and for $R$-almost every $z \in \mathcal{Z}, \gamma^{*}(z, \cdot)$ is a lower semicontinuous strictly convex $[0,+\infty]$-valued function on $\mathbb{R}$.

(2) It is also assumed that for $R$-almost every $z \in \mathcal{Z}, \gamma^{*}(z, \cdot)$ attains a unique minimum denoted by $m(z)$, the minimum value is $\gamma_{z}^{*}(m(z))=0, \forall z \in \mathcal{Z}$ and there exist $a(z), b(z)>0$ such that $0<\gamma^{*}(z, m(z)+a(z))<\infty$ and $0<\gamma^{*}(z, m(z)-b(z))<\infty$.

(3) $\int_{\mathcal{Z}} \gamma^{*}((1+\alpha) m) \mathrm{d} R+\int_{\mathcal{Z}} \gamma^{*}((1-\alpha) m) \mathrm{d} R<\infty$, for some $\alpha>0$.

The function $\gamma_{z}^{*}$ is the convex conjugate of the lower semicontinuous convex function $\gamma_{z}=\gamma_{z}^{* *}$. Defining

$$
\lambda(z, s)=\gamma(z, s)-m(z) s, \quad z \in \mathcal{Z}, s \in \mathbb{R}
$$

we see that for $R$-a.e. $z, \lambda_{z}$ is a nonnegative convex function and it vanishes at $s=0$. 
$\left(\mathrm{A}_{\theta}\right)$ Assumptions on $\theta$.

(1) for any $y \in \mathcal{Y}_{o}$, the function $z \in \mathcal{Z} \mapsto\langle y, \theta(z)\rangle \in \mathbb{R}$ is measurable;

(2) for any $y \in \mathcal{Y}_{o},\langle y, \theta(\cdot)\rangle=0$, $R$-a.e. implies that $y=0$;

( $\exists) \forall y \in \mathcal{Y}_{o}, \exists \alpha>0, \quad \int_{\mathcal{Z}} \lambda(\alpha\langle y, \theta\rangle) \mathrm{d} R<\infty$.

Since

$$
\lambda_{\diamond}(z, s)=\max [\lambda(z, s), \lambda(z,-s)] \in[0, \infty], \quad z \in \mathcal{Z}, s \in \mathbb{R}
$$

is a Young function, one can consider the corresponding Orlicz spaces $L_{\lambda_{\diamond}}$ and $L_{\lambda_{\diamond}^{*}}$ where $\lambda_{\diamond}^{*}(z, \cdot)$ is the convex conjugate of $\lambda_{\diamond}(z, \cdot)$.

Remark 2.5 (some comments about these assumptions).

(1) Assuming $R$ to be $\sigma$-finite is standard when working with integral functionals. Here, $R$ is assumed to be bounded for simplicity.

(2) Thanks to the theory of normal integrands [25], $\gamma=\gamma^{* *}$ is jointly measurable.

The function $m$ is measurable since its graph $\{(z, u): u=m(z)\}$ is measurable. Indeed, it is the $(z, u)$-projection of the intersection of the horizontal hyperplane $u=0$ with the measurable graph $\left\{(t, z, u): \gamma_{z}^{*}(t)=u\right\}$ of $\gamma^{*}$. Note that we use the uniqueness assumption in $\left(\mathrm{A}_{\gamma^{*}}^{2}\right)$ to avoid a measurable selection argument.

As a consequence, $\lambda$ is a jointly measurable function.

(3) The assumption $\left(\mathrm{A}_{\gamma^{*}}^{3}\right)$ implies that $m$ is in the Orlicz space $L_{\lambda_{\diamond}^{*}}$, see (2.5). In turn, the centered form $\ell-m R$ is in $L_{\lambda_{\diamond}}^{\prime}$ whenever $\ell$ is. This will be used later without warning. It also follows from (2.4) that $\gamma_{z}^{*}(t)=\lambda_{z}^{*}(t-m(z))$. These two facts allow us to write

$$
I_{\gamma^{*}}(f R)=I_{\lambda^{*}}(f R-m R), \quad f \in L_{\lambda_{\diamond}^{*}}
$$

where $\lambda_{z}^{*}$ is the convex conjugate of $\lambda_{z}$.

(4) The assumption $\left(\mathrm{A}_{\theta}^{\exists}\right)$ is equivalent to $\langle y, \theta(\cdot)\rangle \in L_{\lambda_{\diamond}}$ for all $y \in \mathcal{Y}_{o}$, see (2.7) below.

\subsection{Examples}

We consider the entropies which were introduced after (1.7) and give the corresponding functions $\lambda$ and $\lambda_{\diamond}$.

(1) The relative entropy: $\lambda(s)=\mathrm{e}^{s}-s-1, \lambda_{\diamond}(s)=\lambda(|s|)$.

(2) The reverse relative entropy: $\lambda(s)=-\log (1-s)-s+\iota_{\{s<1\}}, \lambda_{\diamond}(s)=\lambda(|s|)$.

(3) The Fermi-Dirac entropy: $\lambda(s)=\lambda_{\diamond}(s)=\log \cosh s$.

(4) The $L_{p}$ norm $(1<p<\infty): \lambda(s)=\lambda_{\diamond}(s)=|s|^{q} / q$ with $1 / p+1 / q=1$ and

(5) the $L_{p}$ entropy $(1<p<\infty): \lambda(s)= \begin{cases}0 & \text { if } s \leq 0 \quad \lambda_{\diamond}(s)=|s|^{q} / q . \\ s^{q} / q & \text { if } s \geq 0,\end{cases}$

\subsection{The entropy minimization problems $\left(\mathbf{P}_{C}\right)$ and $\left(\overline{\mathbf{P}}_{C}\right)$}

Because of the Remark 2.5(3), under $\left(\mathrm{A}_{\gamma^{*}}^{3}\right)$ the effective domain of $I$ given at (1.5) is included in $L_{\lambda_{\diamond}^{*}} R$ and the entropy functional to be considered is defined by

$$
I(f R)=\int_{\mathcal{Z}} \gamma^{*}(f) \mathrm{d} R, \quad f \in L_{\lambda_{\triangleright}^{*}} .
$$

Assuming $\left(\mathrm{A}_{\theta}\right)$, that is

$$
\left\langle\mathcal{Y}_{o}, \theta(\cdot)\right\rangle \subset L_{\lambda_{\diamond}}
$$

Hölder's inequality in Orlicz spaces, see (2.1), allows to define the constraint operator $T: \ell \in L_{\lambda_{\diamond}}^{\prime} \mapsto\langle\theta, \ell\rangle \in$ $\mathcal{X}_{o}$ by:

$$
\langle y,\langle\theta, \ell\rangle\rangle_{\mathcal{Y}_{o}, \mathcal{X}_{o}}=\langle\langle y, \theta\rangle, \ell\rangle_{L_{\lambda_{\diamond}}, L_{\lambda_{\diamond}}^{\prime}}, \forall y \in \mathcal{Y}_{o}
$$

If $\ell=Q \in L_{\lambda_{\diamond}^{*}} R \subset M_{\mathcal{Z}}$, one writes $T Q=\langle\theta, Q\rangle=\int_{\mathcal{Z}} \theta \mathrm{d} Q$ to mean (2.8); this is a $\mathcal{X}_{o^{-}}$-valued weak integral. 
Example 2.6 (moment constraint). This is the easiest constraint to think of. Let $\theta=\left(\theta_{k}\right)_{1 \leq k \leq K}$ be a measurable function from $\mathcal{Z}$ to $\mathcal{X}_{o}=\mathbb{R}^{K}$. The moment constraint is specified by the operator

$$
\int_{\mathcal{Z}} \theta \mathrm{d} \ell=\left(\int_{\mathcal{Z}} \theta_{k} \mathrm{~d} \ell\right)_{1 \leq k \leq K} \in \mathbb{R}^{K}
$$

which is defined for each $\ell \in M_{\mathcal{Z}}$ which integrates all the real valued measurable functions $\theta_{k}$.

The minimization problem (1.6) becomes

$$
\text { minimize } I(Q) \text { subject to } \int_{\mathcal{Z}} \theta \mathrm{d} Q \in C, \quad Q \in L_{\lambda_{\triangleright}^{*}} R
$$

where $C$ is a convex subset of $\mathcal{X}_{o}$. The extended entropy is defined by

$$
\bar{I}(\ell)=I\left(\ell^{a}\right)+I^{s}\left(\ell^{s}\right), \quad \ell \in L_{\lambda_{\diamond}}^{\prime}
$$

where, using the notation of Theorem 2.4,

$$
I^{s}\left(\ell^{s}\right)=\iota_{\operatorname{dom} I_{\gamma}}^{*}\left(\ell^{s}\right)=\sup \left\{\left\langle\ell^{s}, u\right\rangle ; u \in L_{\lambda_{\diamond}}, I_{\gamma}(u)<\infty\right\} \in[0, \infty] .
$$

It is proved in [18] that $\bar{I}$ is the greatest convex $\sigma\left(L_{\lambda_{\diamond}}^{\prime}, L_{\lambda_{\diamond}}\right)$-lower semicontinuous extension of $I$ to $L_{\lambda_{\diamond}}^{\prime} \supset L_{\lambda_{\diamond}^{*}} R$. The associated extended minimization problem is

$$
\text { minimize } \bar{I}(\ell) \text { subject to }\langle\theta, \ell\rangle \in C, \quad \ell \in L_{\lambda_{\diamond}}^{\prime} \text {. }
$$

\subsection{Good and critical constraints}

If the Young function $\lambda_{\diamond}$ doesn't satisfy the $\Delta_{2}$-condition (2.3) as for instance with the relative entropy and the reverse relative entropy (see items (1) and (2) of the examples above), the small Orlicz space $E_{\lambda_{\diamond}}$ may be a proper subset of $L_{\lambda_{\diamond}}$. Consequently, for some functions $\theta$, the integrability property

$$
\left\langle\mathcal{Y}_{o}, \theta(\cdot)\right\rangle \subset E_{\lambda_{\diamond}}
$$

or equivalently

$$
\forall y \in \mathcal{Y}_{o}, \int_{\mathcal{Z}} \lambda(\langle y, \theta\rangle) \mathrm{d} R<\infty
$$

may not be satisfied while the weaker property

$$
\forall y \in \mathcal{Y}_{o}, \exists \alpha>0, \int_{\mathcal{Z}} \lambda(\alpha\langle y, \theta\rangle) \mathrm{d} R<\infty
$$

which is equivalent to (2.7), holds. In this situation, analytical complications occur, see Section 4. This is the reason why constraint satisfying $\left(\mathrm{A}_{\theta}^{\forall}\right)$ are called good constraints, while constraints satisfying $\left(\mathrm{A}_{\theta}^{\exists}\right)$ but not $\left(\mathrm{A}_{\theta}^{\forall}\right)$ are called critical constraints.

\subsection{Definitions of $\mathcal{Y}, \mathcal{X}, T^{*}, \Gamma^{*}$ and $\left(\mathrm{D}_{C}\right)$}

These objects will be necessary to state the relevant dual problems. The general hypotheses (A) are assumed.

The space $\mathcal{Y}$. Because of the hypotheses $\left(\mathrm{A}_{\theta}^{2}\right)$ and $\left(\mathrm{A}_{\theta}^{\exists}\right), \mathcal{Y}_{o}$ can be identified with the subspace $\left\langle\mathcal{Y}_{o}, \theta(\cdot)\right\rangle$ of $L_{\lambda_{\diamond}}$. The space $\mathcal{Y}$ is the extension of $\mathcal{Y}_{o}$ which is isomorphic to the $\|\cdot\|_{\lambda_{\diamond}}$-closure of $\left\langle\mathcal{Y}_{o}, \theta(\cdot)\right\rangle$ in $L_{\lambda_{\diamond}}$. 
The space $\mathcal{X}$. The topological dual space of $\mathcal{Y}$ is $\mathcal{X}=\mathcal{Y}^{\prime} \subset \mathcal{X}_{o} . \mathcal{X}$ is identified with $L_{\lambda_{\diamond}}^{\prime} / \operatorname{ker} T$.

Since $\lambda_{\diamond}$ is finite, we can apply Proposition 2.3. It tells us that under the assumption $\left(\mathrm{A}_{\theta}^{\forall}\right), T L_{\lambda_{\diamond}}^{s}=\{0\}$ so that $\mathcal{X} \cong L_{\lambda_{0}^{*}} R / \operatorname{ker} T$.

The operator $T^{*}$. Let us define the adjoint $T^{\sharp}: \mathcal{X}^{*} \rightarrow L_{\lambda_{\diamond}}^{\prime *}$ for all $\omega \in \mathcal{X}^{*}$ by: $\left\langle\ell, T^{\sharp} \omega\right\rangle_{L_{\lambda_{\diamond}}^{\prime}, L_{\lambda_{\diamond}}^{\prime *}}=\langle T \ell, \omega\rangle_{\mathcal{X}, \mathcal{X}^{*}}, \forall \ell \in$ $L_{\lambda_{\diamond}}^{\prime}$. We have the inclusions $\mathcal{Y}_{o} \subset \mathcal{Y} \subset \mathcal{X}^{*}$. The adjoint operator $T^{*}$ is the restriction of $T^{\sharp}$ to $\mathcal{Y}_{o}$. With some abuse of notation, we still denote $T^{*} y=\langle y, \theta\rangle$ for $y \in \mathcal{Y}$. This can be interpreted as a dual bracket between $\mathcal{X}_{o}^{*}$ and $\mathcal{X}_{o}$ since $T^{*} y=\langle\tilde{y}, \theta\rangle R$-a.e. for some $\tilde{y} \in \mathcal{X}_{o}^{*}$.

The function $\Gamma^{*}$. The basic dual problem associated with $\left(\mathrm{P}_{C}\right)$ and $\left(\overline{\mathrm{P}}_{C}\right)$ is

$$
\text { maximize } \inf _{x \in C}\langle y, x\rangle-\Gamma(y), \quad y \in \mathcal{Y}_{o}
$$

where

Let us denote

$$
\Gamma(y)=I_{\gamma}(\langle y, \theta\rangle), \quad y \in \mathcal{Y}_{o} .
$$

$$
\Gamma^{*}(x)=\sup _{y \in \mathcal{Y}_{o}}\left\{\langle y, x\rangle-I_{\gamma}(\langle y, \theta\rangle)\right\}, \quad x \in \mathcal{X}_{o}
$$

its convex conjugate. It is shown in [16] (Sect. 4), that $\operatorname{dom} \Gamma^{*} \subset \mathcal{X}$.

The dual problem $\left(\mathrm{D}_{C}\right)$. Another dual problem associated with $\left(\mathrm{P}_{C}\right)$ and $\left(\overline{\mathrm{P}}_{C}\right)$ is

$$
\text { maximize } \inf _{x \in C \cap \mathcal{X}}\langle y, x\rangle-I_{\gamma}(\langle y, \theta\rangle), \quad y \in \mathcal{Y} \text {. }
$$

\subsection{Solving $\left(\mathbf{P}_{C}\right)$}

In this section, we study $\left(\mathrm{P}_{C}\right)$ under the good constraint hypothesis $\left(\mathrm{A}_{\theta}^{\forall}\right)$ which imposes that $T^{*} \mathcal{Y} \subset E_{\lambda_{\diamond}}$ and $T\left(L_{\lambda_{0}^{*}} R\right) \subset \mathcal{X}$.

The extended dual problem $\left(\widetilde{\mathrm{D}}_{C}\right)$. The extended dual problem associated with $\left(\mathrm{P}_{C}\right)$ is

$$
\operatorname{maximize} \inf _{x \in C \cap \mathcal{X}}\langle\omega, x\rangle-I_{\gamma}(\langle\omega, \theta\rangle), \quad \omega \in \widetilde{\mathcal{Y}}
$$

where $\widetilde{\mathcal{Y}} \subset \mathcal{X}^{*}$ is some cone which contains $\mathcal{Y}_{o}$. Its exact definition is given at Appendix A.

Theorem 2.7. Suppose that

(1) the hypotheses $(\mathrm{A})$ and $\left(\mathrm{A}_{\theta}^{\forall}\right)$ are satisfied;

(2) the convex set $C$ is assumed to be such that

$$
T^{-1} C \cap L_{\lambda_{\triangleright}^{*}} R=\bigcap_{y \in Y}\left\{f R \in L_{\lambda_{o}^{*}} R ; \int_{\mathcal{Z}}\langle y, \theta\rangle f \mathrm{~d} R \geq a_{y}\right\}
$$

for some subset $Y \in \mathcal{X}_{o}^{*}$ with $\langle y, \theta\rangle \in E_{\lambda_{\diamond}}$ for all $y \in Y$ and some function $y \in Y \mapsto a_{y} \in \mathbb{R}$. In other words, $T^{-1} C \cap L_{\lambda_{\diamond}^{*}} R$ is a $\sigma\left(L_{\lambda_{\diamond}^{*}} R, E_{\lambda_{\diamond}}\right)$-closed convex subset of $L_{\lambda_{\diamond}^{*}} R$.

Then:

(a) The dual equality for $\left(\mathrm{P}_{C}\right)$ is

$$
\inf \left(\mathrm{P}_{C}\right)=\sup \left(\mathrm{D}_{C}\right)=\sup \left(\widetilde{\mathrm{D}}_{C}\right)=\inf _{x \in C} \Gamma^{*}(x) \in[0, \infty]
$$

(b) If $C \cap \operatorname{dom} \Gamma^{*} \neq \emptyset$ or equivalently $C \cap T \operatorname{dom} I \neq \emptyset$, then $\left(\mathrm{P}_{C}\right)$ admits a unique solution $\widehat{Q}$ in $L_{\lambda_{\diamond}^{*}} R$ and any minimizing sequence $\left(Q_{n}\right)_{n \geq 1}$ converges to $\widehat{Q}$ with respect to the topology $\sigma\left(L_{\lambda_{\diamond}^{*}} R, L_{\lambda_{\diamond}}\right)$. 


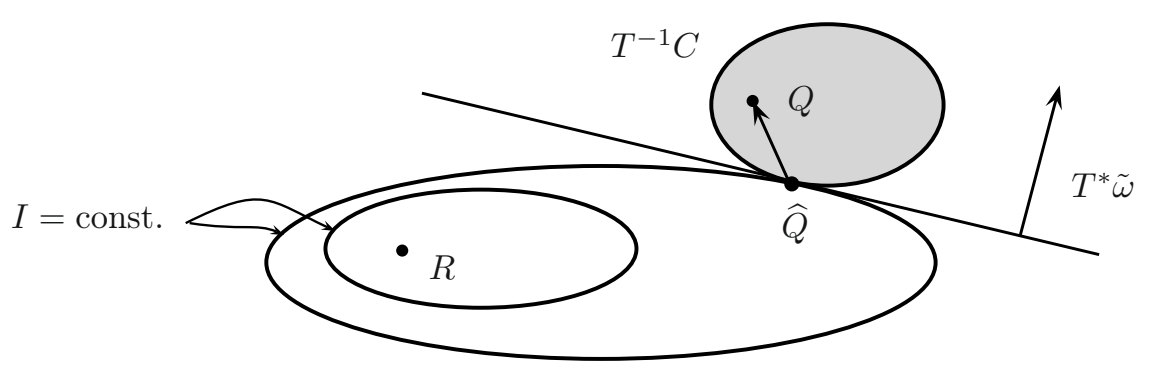

FiguRe 1.

Suppose that in addition $C \cap \operatorname{icordom} \Gamma^{*} \neq \emptyset$ or equivalently $C \cap \operatorname{icor}(T \operatorname{dom} I) \neq \emptyset$.

(c) Let us define $\hat{x}:=\int_{\mathcal{Z}} \theta \mathrm{d} \widehat{Q}$ in the weak sense with respect to the duality $\left\langle\mathcal{Y}_{o}, \mathcal{X}_{o}\right\rangle$. There exists $\tilde{\omega} \in \tilde{\mathcal{Y}}$ such that

$$
\begin{cases}(a) & \hat{x} \in C \cap \operatorname{dom} \Gamma^{*} \\ (b) & \langle\tilde{\omega}, \hat{x}\rangle_{\mathcal{X}^{*}, \mathcal{X}} \leq\langle\tilde{\omega}, x\rangle_{\mathcal{X}}, \mathcal{X}, \forall x \in C \cap \operatorname{dom} \Gamma^{*} \\ (c) & \widehat{Q}(\mathrm{~d} z)=\gamma_{z}^{\prime}(\langle\tilde{\omega}, \theta(z)\rangle) R(\mathrm{~d} z) .\end{cases}
$$

Furthermore, $\widehat{Q} \in L_{\lambda_{\diamond}^{*}} R$ and $\tilde{\omega} \in \widetilde{\mathcal{Y}}$ satisfy $(2.15)$ if and only if $\widehat{Q}$ solves $\left(\mathrm{P}_{C}\right)$ and $\tilde{\omega}$ solves $\left(\widetilde{\mathrm{D}}_{C}\right)$.

(d) Of course, (2.15)c implies

$$
\hat{x}=\int_{\mathcal{Z}} \theta \gamma^{\prime}(\langle\tilde{\omega}, \theta\rangle) \mathrm{d} R
$$

in the weak sense. Moreover,

1. $\hat{x}$ minimizes $\Gamma^{*}$ on $C$;

2. $I(\widehat{Q})=\Gamma^{*}(\hat{x})=\int_{\mathcal{Z}} \gamma^{*} \circ \gamma^{\prime}(\langle\tilde{\omega}, \theta\rangle) \mathrm{d} R<\infty$; and

3. $I(\widehat{Q})+\int_{\mathcal{Z}} \gamma(\langle\tilde{\omega}, \theta\rangle) \mathrm{d} R=\int_{\mathcal{Z}}\langle\tilde{\omega}, \theta\rangle \mathrm{d} \widehat{Q}$.

Proof. This result is [19] (Thm. 3.2).

Figure 1 illustrates the items (a) and (b) of (2.15) which, with $T \widehat{Q}=\hat{x}, T Q=x \in C$, can be rewritten: $\widehat{Q} \in T^{-1} C$ and $\left\langle T^{*} \tilde{\omega}, Q-\widehat{Q}\right\rangle \geq 0, \forall Q \in T^{-1} C$ : the shaded area.

As $R$ is the unconstrained minimizer of $I$, the level sets of $I$ form an increasing family of convex sets which expends from $R$. One sees that the hyperplane $\left\{Q:\left\langle T^{*} \tilde{\omega}, Q-\widehat{Q}\right\rangle=0\right\}$ separates the convex set $T^{-1} C$ and the convex level set of $I$ which is "tangent" to $T^{-1} C$. Informally, the gradient of $I$ at $\widehat{Q}$ is a normal vector of $T^{-1} C$ at $\widehat{Q}$ : it must be a multiple of $T^{*} \tilde{\omega}$. Expressing this orthogonality relation by means of the convex conjugation (2.13) leads to the representation formula (2.15)(c). Clearly, Hahn-Banach theorem has something to do with the existence of $T^{*} \tilde{\omega}$. This picture is a guide for the proof in [19].

Following the terminology of Ney [22,23], as it shares the properties $(2.15)(\mathrm{a}, \mathrm{b})$ and $(2.16)$, the minimizer $\hat{x}$ is called a dominating point of $C$ with respect to $\Gamma^{*}$ (see Def. 6.1 below).

\subsection{Solving $\left(\overline{\mathbf{P}}_{C}\right)$}

In this section, we study $\left(\overline{\mathrm{P}}_{C}\right)$ under the critical constraint hypothesis $\left(\mathrm{A}_{\theta}^{\exists}\right)$ which imposes that $T^{*} \mathcal{Y} \subset L_{\lambda_{\diamond}}$.

By Theorem 2.4, we have $L_{\rho}^{\prime \prime}=\left[L_{\rho} \oplus L_{\rho^{*}}^{s} \oplus L_{\rho}^{s \prime}\right.$. For any $\zeta \in L_{\rho}^{\prime \prime}=\left(L_{\rho^{*}} R \oplus L_{\rho}^{s}\right)^{\prime}$, let us denote the restrictions $\zeta_{1}=\zeta_{\mid L_{\rho^{*}} R}$ and $\zeta_{2}=\zeta_{\mid L_{\rho}^{s}}$. Since, $\left(L_{\rho^{*}} R\right)^{\prime} \simeq L_{\rho} \oplus L_{\rho^{*}}^{s}$, we see that any $\zeta \in L_{\rho}^{\prime \prime}$ is uniquely decomposed into

$$
\zeta=\zeta_{1}^{a}+\zeta_{1}^{s}+\zeta_{2}
$$

with $\zeta_{1}=\zeta_{1}^{a}+\zeta_{1}^{s} \in L_{\rho^{*}}^{\prime}, \zeta_{1}^{a} \in L_{\rho}, \zeta_{1}^{s} \in L_{\rho^{*}}^{s}$ and $\zeta_{2} \in L_{\rho}^{s \prime}$ 
The extended dual problem $\left(\overline{\mathrm{D}}_{C}\right)$. The extended dual problem associated with $\left(\overline{\mathrm{P}}_{C}\right)$ is

$$
\text { maximize } \inf _{x \in C \cap \mathcal{X}}\langle\omega, x\rangle-I_{\gamma}\left(\left[T^{*} \omega\right]_{1}^{a}\right), \quad \omega \in \overline{\mathcal{Y}}
$$

where $\overline{\mathcal{Y}} \subset \mathcal{X}^{*}$ is some cone which contains $\mathcal{Y}_{o}$. Its exact definition is given at Appendix A.

Theorem 2.8. Suppose that

(1) the hypotheses (A) are satisfied,

(2) the convex set $C$ is assumed to be such that

$$
T^{-1} C \cap L_{\lambda_{\diamond}}^{\prime}=\bigcap_{y \in Y}\left\{\ell \in L_{\lambda_{\diamond}}^{\prime} ;\langle\langle y, \theta\rangle, \ell\rangle \geq a_{y}\right\}
$$

for some subset $Y \subset \mathcal{X}_{o}^{*}$ with $\langle y, \theta\rangle \in L_{\lambda_{\diamond}}$ for all $y \in Y$ and some function $y \in Y \mapsto a_{y} \in \mathbb{R}$. In other Then: words, $T^{-1} C$ is a $\sigma\left(L_{\lambda_{\diamond}}^{\prime}, L_{\lambda_{\diamond}}\right)$-closed convex subset of $L_{\lambda_{\diamond}}^{\prime}$.

(a) The dual equality for $\left(\overline{\mathrm{P}}_{C}\right)$ is

$$
\inf \left(\overline{\mathrm{P}}_{C}\right)=\inf _{x \in C} \Gamma^{*}(x)=\sup \left(\mathrm{D}_{C}\right)=\sup \left(\overline{\mathrm{D}}_{C}\right) \in[0, \infty]
$$

(b) If $C \cap \operatorname{dom} \Gamma^{*} \neq \emptyset$ or equivalently $C \cap T \operatorname{dom} \bar{I} \neq \emptyset$, then $\left(\overline{\mathrm{P}}_{C}\right)$ admits solutions in $L_{\lambda_{\diamond}}^{\prime}$, any minimizing sequence admits $\sigma\left(L_{\lambda_{\odot}}^{\prime}, L_{\lambda_{\diamond}}\right)$-cluster points and every such point is a solution to $\left(\overline{\mathrm{P}}_{C}\right)$.

Suppose that in addition we have $C \cap \operatorname{icordom} \Gamma^{*} \neq \emptyset$ or equivalently $C \cap \operatorname{icor}(T \operatorname{dom} \bar{I}) \neq \emptyset$. Then:

(c) Let $\hat{\ell} \in L_{\lambda_{\diamond}}^{\prime}$ be any solution to $\left(\overline{\mathrm{P}}_{C}\right)$ and denote $\hat{x}:=$ The There exists $\bar{\omega} \in \overline{\mathcal{Y}}$ such that

$$
\begin{cases}(a) & \hat{x} \in C \cap \operatorname{dom} \Gamma^{*} \\ (b) & \langle\bar{\omega}, \hat{x}\rangle_{\mathcal{X}^{*}, \mathcal{X}} \leq\langle\bar{\omega}, x\rangle_{\mathcal{X}^{*}, \mathcal{X}}, \forall x \in C \cap \operatorname{dom} \Gamma^{*} \\ (c) & \hat{\ell} \in \gamma_{z}^{\prime}\left(\left[T^{*} \bar{\omega}\right]_{1}^{a}\right) R+D^{\perp}\left(\left[T^{*} \bar{\omega}\right]_{2}\right)\end{cases}
$$

where we used notation (2.17) and $D^{\perp}\left(\left[T^{*} \bar{\omega}\right]_{2}\right)$ is some cone in $L_{\lambda_{\diamond}}^{s}$ which is pointed at zero and whose direction depends on $\left[T^{*} \bar{\omega}\right]_{2}$ (see Appendix A for its precise definition).

There exists some $\tilde{\omega} \in \mathcal{X}_{0}^{*}$ such that

$$
\left[T^{*} \bar{\omega}\right]_{1}^{a}=\langle\tilde{\omega}, \theta(\cdot)\rangle_{\mathcal{X}_{o}^{*}, \mathcal{X}_{o}}
$$

is a measurable function which can be approximated in some sense (see Appendix A) by sequences $\left(\left\langle y_{n}, \theta(\cdot)\right\rangle\right)_{n \geq 1}$ with $y_{n} \in \mathcal{Y}_{o}$ and $\int_{\mathcal{Z}} \lambda\left(\left\langle y_{n}, \theta\right\rangle\right) d R<\infty$ for all $n$.

Furthermore, $\hat{\ell} \in L_{\lambda_{\diamond}}^{\prime}$ and $\bar{\omega} \in \overline{\mathcal{Y}}$ satisfy (2.19) if and only if $\hat{\ell}$ solves $\left(\overline{\mathrm{P}}_{C}\right)$ and $\bar{\omega}$ solves $\left(\overline{\mathrm{D}}_{C}\right)$.

(d) Of course, (2.19)c implies $\hat{x}=\int_{\mathcal{Z}} \theta \gamma^{\prime}(\langle\tilde{\omega}, \theta\rangle) \mathrm{d} R+\left\langle\theta, \hat{\ell}^{s}\right\rangle$. Moreover,
1. $\hat{x}$ minimizes $\Gamma^{*}$ on $C$,
2. $\bar{I}(\hat{\ell})=\Gamma^{*}(\hat{x})=\int_{\mathcal{Z}} \gamma^{*} \circ \gamma^{\prime}(\langle\tilde{\omega}, \theta\rangle) \mathrm{d} R+\sup \left\{\left\langle u, \hat{\ell}^{s}\right\rangle ; u \in \operatorname{dom} I_{\gamma}\right\}<\infty$ and
3. $\bar{I}(\hat{\ell})+\int_{\mathcal{Z}} \gamma(\langle\tilde{\omega}, \theta\rangle) \mathrm{d} R=\int_{\mathcal{Z}}\langle\tilde{\omega}, \theta\rangle \mathrm{d} \hat{\ell}^{a}+\left\langle\left[T^{*} \bar{\omega}\right]_{2}, \hat{\ell}^{s}\right\rangle$.

Proof. This result is [19] (Thm. 4.2).

The exact definitions of $\tilde{\mathcal{Y}}, \overline{\mathcal{Y}}$ and $D^{\perp}$ as well as the precise statement of Theorem 2.8(c) are given at Appendix A. In particular, the complete statement of Theorem 2.8(c) is given at Theorem A.1.

Figure 2 illustrates (2.19) which, with $\hat{x}=T \hat{\ell}$ can be rewritten: $\hat{\ell} \in T^{-1} C$ and $\left\langle T^{*} \bar{\omega}, \ell-\hat{\ell}\right\rangle \geq 0, \forall \ell \in T^{-1} C$. As in Figure 1, one sees that the hyperplane $\left\{\ell:\left\langle T^{*} \bar{\omega}, \ell-\hat{\ell}\right\rangle=0\right\}$ separates the convex set $T^{-1} C$ and the convex level set of $\bar{I}$ which is "tangent" to $T^{-1} C$ : the shaded area. The same kind of conclusions follow. 


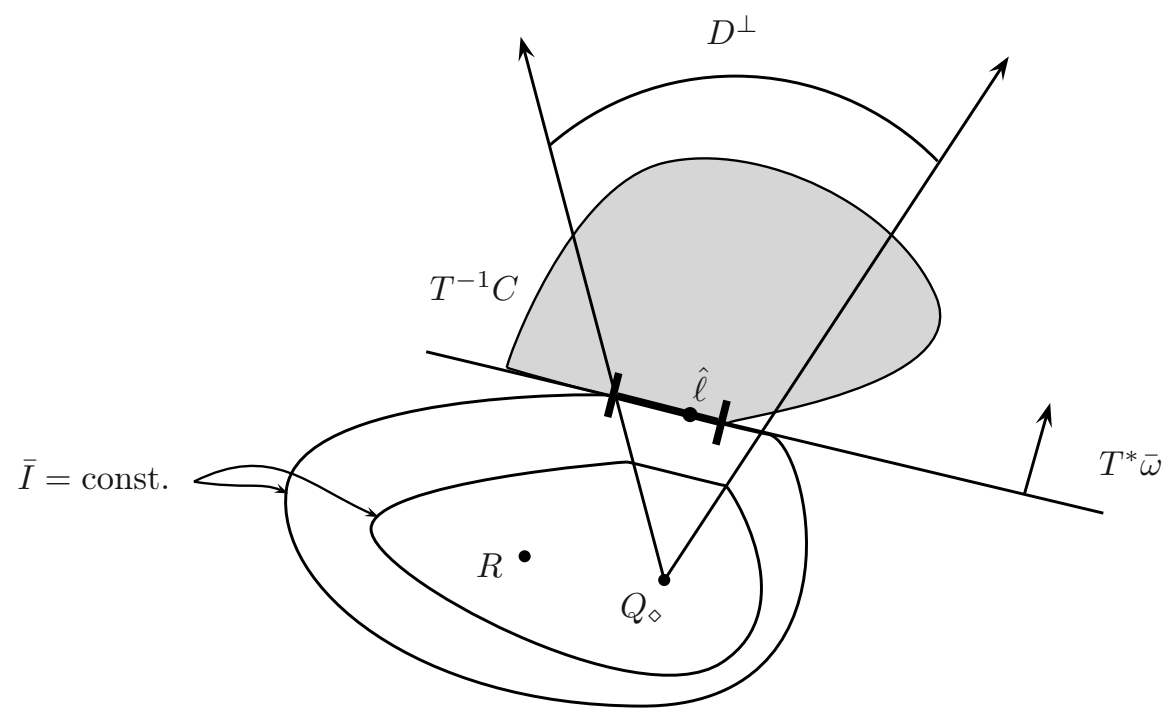

FiguRE 2 .

The measure $Q_{\diamond}=\hat{\ell}^{a}=\gamma_{z}^{\prime}\left(\left[T^{*} \bar{\omega}\right]_{1}^{a}\right) R$ is the absolutely continuous part of $\hat{\ell}$; its expression is given at (4.1) below. The set of solutions $\hat{\ell}$ of $\left(\overline{\mathrm{P}}_{C}\right)$ is represented by the bold type segment, they all share the same absolutely continuous part $Q_{\diamond}$. The relation $(2.19)(\mathrm{c})$ can be rewritten: $\hat{\ell} \in Q_{\diamond}+D^{\perp}$ which is the convex cone with vertex $Q_{\diamond}$ and direction $D^{\perp}$. The cone $D^{\perp}$ only contains singular directions. One sees with $(2.9)$ and $(2.10)$ that $D^{\perp}$ contributes to the positively homogeneous part $I^{s}$ of $\bar{I}$. This is the reason why we decided to draw a flat part for the level lines of $\bar{I}$ when they cut $Q_{\diamond}+D^{\perp}$.

Figure 2 is only a guide for illustrating Theorem 2.8. It shouldn't be taken too seriously. For instance a better finite dimensional analogue would have been given by $\bar{I}(x, y, z)=x^{2}+|y+z|$ which requires a 3D graphical representation.

\section{SOME EXAMPLES}

\subsection{Some examples of entropies}

Important examples of entropies occur in statistical physics, probability theory and mathematical statistics. Among them the relative entropy plays a prominent role.

\section{Relative entropy}

The reference measure $R$ is assumed to be a probability measure. The relative entropy of $Q \in M_{\mathcal{Z}}$ with respect to $R \in P_{\mathcal{Z}}$ is

$$
I(Q \mid R)=\left\{\begin{array}{ll}
\int_{\mathcal{Z}} \log \left(\frac{\mathrm{d} Q}{\mathrm{~d} R}\right) \mathrm{d} Q & \text { if } Q \prec R \text { and } Q \in P_{\mathcal{Z}} \\
+\infty & \text { otherwise, }
\end{array} \quad Q \in M_{\mathcal{Z}} .\right.
$$

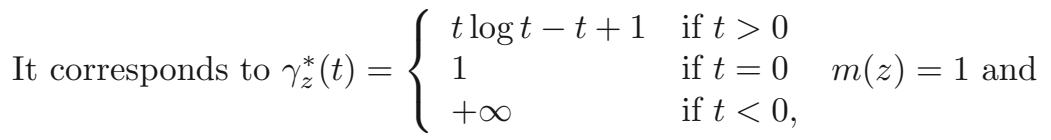

$$
\lambda_{z}(s)=\mathrm{e}^{s}-s-1, \quad s \in \mathbb{R}, z \in \mathcal{Z} .
$$




\section{A variant}

Taking the same $\gamma^{*}$ and removing the unit mass constraint gives

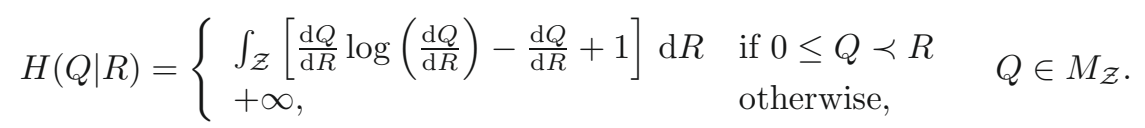

This entropy is the rate function of (1.4) when $\left(W_{i}\right)_{i \geq 1}$ is an iid sequence of Poisson (1) random weights. If $R$ is $\sigma$-finite, it is the rate function of the LDP of normalized Poisson random measures, see [15].

\section{Extended relative entropy}

Since $\lambda(s)=\mathrm{e}^{s}-s-1$ and $R \in P_{\mathcal{Z}}$ is a bounded measure, we have $\lambda_{\diamond}(s)=\tau(s):=\mathrm{e}^{|s|}-|s|-1$ and the relevant Orlicz spaces are

$$
\begin{aligned}
L_{\tau^{*}} & =\left\{f: \mathcal{Z} \rightarrow \mathbb{R} ; \int_{\mathcal{Z}}|f| \log |f| \mathrm{d} R<\infty\right\} \\
E_{\tau} & =\left\{u: \mathcal{Z} \rightarrow \mathbb{R} ; \forall \alpha>0, \int_{\mathcal{Z}} \mathrm{e}^{\alpha|u|} \mathrm{d} R<\infty\right\} \\
L_{\tau} & =\left\{u: \mathcal{Z} \rightarrow \mathbb{R} ; \exists \alpha>0, \int_{\mathcal{Z}} \mathrm{e}^{\alpha|u|} \mathrm{d} R<\infty\right\}
\end{aligned}
$$

since $\tau^{*}(t)=(|t|+1) \log (|t|+1)-|t|$. The extended relative entropy is defined by

$$
\bar{I}(\ell \mid R)=I\left(\ell^{a} \mid R\right)+\sup \left\{\left\langle\ell^{s}, u\right\rangle ; u, \int_{\mathcal{Z}} \mathrm{e}^{u} d R<\infty\right\}, \quad \ell \in O_{\exp }
$$

where $\ell=\ell^{a}+\ell^{s}$ is the decomposition into absolutely continuous and singular parts of $\ell$ in $L_{\tau}^{\prime}=L_{\tau^{*}} \oplus L_{\tau}^{s}$, and

$$
O_{\exp }=\left\{\ell \in L_{\tau}^{\prime} ; \ell \geq 0,\langle\ell, \mathbf{1}\rangle=1\right\}
$$

Note that $O_{\exp }$ depends on $R$ and that for all $\ell \in O_{\exp }, \ell^{a} \in P_{\mathcal{Z}} \cap L_{\tau^{*}} R$.

\subsection{Some examples of constraints}

Let us consider the two standard constraints which are the moment constraints and the marginal constraints.

\section{Moment constraints}

Let $\theta=\left(\theta_{k}\right)_{1 \leq k \leq K}$ be a measurable function from $\mathcal{Z}$ to $\mathcal{X}_{o}=\mathbb{R}^{K}$. The moment constraint is specified by the operator

$$
\int_{\mathcal{Z}} \theta \mathrm{d} \ell=\left(\int_{\mathcal{Z}} \theta_{k} \mathrm{~d} \ell\right)_{1 \leq k \leq K} \in \mathbb{R}^{K}
$$

which is defined for each $\ell \in M_{\mathcal{Z}}$ which integrates all the real valued measurable functions $\theta_{k}$. The adjoint operator is

$$
T^{*} y=\langle y, \theta\rangle=\sum_{1 \leq k \leq K} y_{k} \theta_{k}, \quad y=\left(y_{1}, \ldots, y_{K}\right) \in \mathbb{R}^{K}
$$




\section{Marginal constraints}

Let $\mathcal{Z}=A \times B$ be a product space, $M_{A B}$ be the space of all bounded signed measures on $A \times B$ and $U_{A B}$ be the space of all measurable bounded functions $u$ on $A \times B$. Denote $\ell_{A}=\ell(\cdot \times B)$ and $\ell_{B}=\ell(A \times \cdot)$ the marginal measures of $\ell \in M_{A B}$. The constraint of prescribed marginal measures is specified by

$$
\int_{A \times B} \theta \mathrm{d} \ell=\left(\ell_{A}, \ell_{B}\right) \in M_{A} \times M_{B}, \quad \ell \in M_{A B}
$$

where $M_{A}$ and $M_{B}$ are the spaces of all bounded signed measures on $A$ and $B$. The function $\theta$ which gives the marginal constraint is

$$
\theta(a, b)=\left(\delta_{a}, \delta_{b}\right), a \in A, b \in B
$$

where $\delta_{a}$ is the Dirac measure at $a$. Indeed, $\left(\ell_{A}, \ell_{B}\right)=\int_{A \times B}\left(\delta_{a}, \delta_{b}\right) \ell(\mathrm{d} a \mathrm{~d} b)$.

More precisely, let $U_{A}, U_{B}$ be the spaces of measurable functions on $A$ and $B$ and take $\mathcal{Y}_{o}=U_{A} \times U_{B}$ and $\mathcal{X}_{o}=U_{A}^{*} \times U_{B}^{*}$. Then, $\theta$ is a measurable function from $\mathcal{Z}=A \times B$ to $\mathcal{X}_{o}=U_{A}^{*} \times U_{B}^{*}$ and the adjoint of the marginal operator

$$
T \ell=\langle\theta, \ell\rangle=\left(\ell_{A}, \ell_{B}\right) \in U_{A}^{*} \times U_{B}^{*}, \quad \ell \in U_{A B}^{*}
$$

where $\left\langle f, \ell_{A}\right\rangle:=\langle f \otimes 1, \ell\rangle$ and $\left\langle g, \ell_{B}\right\rangle:=\langle 1 \otimes g, \ell\rangle$ for all $f \in U_{A}$ and $g \in U_{B}$, is given by

$$
T^{*}(f, g)=\langle(f, g), \theta\rangle=f \oplus g \in U_{A B}, \quad f \in U_{A}, g \in U_{B}
$$

where $f \oplus g(a, b):=f(a)+g(b), a \in A, b \in B$.

\section{Minimizing SEQuences}

In this section, the minimization problem $\left(\mathrm{P}_{C}\right)$ is considered when the constraint function $\theta$ satisfies $\left(\mathrm{A}_{\theta}^{\exists}\right)$ but not necessarily $\left(\mathrm{A}_{\theta}^{\forall}\right)$. This means that the constraint is critical. Problem $\left(\mathrm{P}_{C}\right)$ may not be attained anymore. Nevertheless, minimizing sequences may admit a limit in some sense. As will be seen at Section 5 , this phenomenon is tightly linked to the notion of generalized entropic projection introduced by Csiszár.

\subsection{Statements of the results}

We start this section stating its main results at Theorems 4.1 and 4.4 .

Theorem 4.1 (attainment). The hypotheses of Theorem 2.8 are assumed.

(a) Suppose that $C \cap \operatorname{dom} \Gamma^{*} \neq \emptyset$. Then, the minimization problem $\left(\overline{\mathrm{P}}_{C}\right)$ is attained in $L_{\lambda_{\diamond}}$ and all its solutions share the same absolutely continuous part $Q_{\diamond} \in L_{\lambda_{\diamond}^{*}} R$.

(b) Suppose that $C \cap \operatorname{icordom} \Gamma^{*} \neq \emptyset$. Then, $\left(\widetilde{\mathrm{D}}_{C}\right)$ is attained in $\widetilde{\mathcal{Y}}$ and

$$
Q_{\diamond}(\mathrm{d} z)=\gamma_{z}^{\prime}\left(\left\langle\omega_{\diamond}, \theta(z)\right\rangle\right) R(\mathrm{~d} z)
$$

where $\omega_{\diamond} \in \widetilde{\mathcal{Y}}$ is any solution to $\left(\widetilde{\mathrm{D}}_{C}\right)$.

(c) See also an additional statement at Proposition A.2.

Proof.

- Proof of (a). The attainment statement is Theorem 2.8b. Let us show that as $\gamma^{*}$ is strictly convex, if $k_{*}$ and $\ell_{*}$ are two solutions of $\left(\overline{\mathrm{P}}_{C}\right)$, their absolutely continuous parts match:

$$
k_{*}^{a}=\ell_{*}^{a} .
$$


$k_{*}, \ell_{*}$ are in the convex set $\left\{\ell \in L_{\lambda_{\diamond}}^{\prime} ; T \ell \in C\right\}$ and $\inf \left(\overline{\mathrm{P}}_{C}\right)=\bar{I}\left(k_{*}\right)=\bar{I}\left(\ell_{*}\right)$. For all $0 \leq p, q \leq 1$ such that $p+q=1$, as $I$ and $I^{s}$ are convex functions, we have

$$
\begin{aligned}
\inf \left(\overline{\mathrm{P}}_{C}\right) & \leq \bar{I}\left(p k_{*}+q \ell_{*}\right) \\
& =I\left(p k_{*}^{a}+q \ell_{*}^{a}\right)+I^{s}\left(p k_{*}^{s}+q \ell_{*}^{s}\right) \\
& \leq p I\left(k_{*}^{a}\right)+q I\left(\ell_{*}^{a}\right)+p I^{s}\left(k_{*}^{s}\right)+q I^{s}\left(\ell_{*}^{s}\right) \\
& =p \bar{I}\left(k_{*}\right)+q \bar{I}\left(\ell_{*}\right)=\inf \left(\overline{\mathrm{P}}_{C}\right) .
\end{aligned}
$$

It follows that $I\left(p k_{*}^{a}+q \ell_{*}^{a}\right)+I^{s}\left(p k_{*}^{s}+q \ell_{*}^{s}\right)=p I\left(k_{*}^{a}\right)+q I\left(\ell_{*}^{a}\right)+p I^{s}\left(k_{*}^{s}\right)+q I^{s}\left(\ell_{*}^{s}\right)$. Suppose that $k_{*}^{a} \neq \ell_{*}^{a}$. As $I$ is strictly convex, with $0<p<1$, we get: $I\left(p k_{*}^{a}+q \ell_{*}^{a}\right)<p I\left(k_{*}^{a}\right)+q I\left(\ell_{*}^{a}\right)$ and this implies that $I^{s}\left(p k_{*}^{s}+q \ell_{*}^{s}\right)>p I^{s}\left(k_{*}^{s}\right)+q I^{s}\left(\ell_{*}^{s}\right)$ which is impossible since $I^{s}$ is convex. This proves (4.2).

- Proof of (b). Let $\bar{\ell}$ be any solution to $\left(\overline{\mathrm{P}}_{C}\right)$. Denoting $\bar{x}^{a}=T \bar{\ell}^{a}$ and $\bar{x}^{s}=T \bar{\ell}^{s}$ we see with (2.19) that

$$
\begin{cases}(a) & \bar{x}^{a} \in\left[C-\bar{x}^{s}\right] \cap \operatorname{dom} \Gamma^{*} \\ (b) & \left\langle\bar{\omega}, \bar{x}^{a}\right\rangle \leq\langle\bar{\omega}, x\rangle, \forall x \in\left[C-\bar{x}^{s}\right] \cap \operatorname{dom} \Gamma^{*} \\ (c) & \bar{\ell}^{a}=\gamma_{z}^{\prime}(\langle\tilde{\omega}, \theta\rangle) R .\end{cases}
$$

By Theorem $2.7 \mathrm{c}$, this implies that $\tilde{\omega}$ solves $\left(\widetilde{\mathrm{D}}_{C-\bar{x}^{s}}\right)$.

It remains to show that $\tilde{\omega}$ also solves $\left(\widetilde{\mathrm{D}}_{C}\right)$. Thanks to Theorem 2.8, we have: $\inf _{x \in C}\langle\bar{\omega}, x\rangle=$ $\left\langle T^{*} \bar{\omega}, \bar{\ell}\right\rangle=\left\langle\langle\tilde{\omega}, \theta\rangle, \bar{\ell}^{a}\right\rangle+\bar{I}\left(\bar{\ell}^{s}\right)$ and $\inf _{x \in C}\langle\bar{\omega}, x\rangle-I_{\gamma}(\langle\tilde{\omega}, \theta\rangle)=\sup \left(\overline{\mathrm{D}}_{C}\right)=\inf \left(\overline{\mathrm{P}}_{C}\right)=I\left(\bar{\ell}^{a}\right)+\bar{I}\left(\bar{\ell}^{s}\right)=$ $\inf \left(\overline{\mathrm{P}}_{C-\bar{x}^{s}}\right)+\bar{I}\left(\bar{\ell}^{s}\right)=\sup \left(\overline{\mathrm{D}}_{C-\bar{x}^{s}}\right)+\bar{I}\left(\bar{\ell}^{s}\right)$. Therefore $\inf _{x \in C}\langle\bar{\omega}, x\rangle=\inf _{x \in C-\bar{x}^{s}}\langle\tilde{\omega}, x\rangle+\bar{I}\left(\bar{\ell}^{s}\right)$ and subtracting $\bar{I}\left(\bar{\ell}^{s}\right)$ from $\sup \left(\overline{\mathrm{D}}_{C}\right)$, we see that $\tilde{\omega}$ which solves $\left(\widetilde{\mathrm{D}}_{C-\bar{x}^{s}}\right)$ also solves $\left(\widetilde{\mathrm{D}}_{C}\right)$. We complete the proof of the theorem, taking $\omega_{\diamond}=\tilde{\omega}$.

Remark 4.2. Replacing $\bar{\ell}^{s}$ with $t \bar{\ell}^{s}$, the same proof shows that $\tilde{\omega}$ solves $\left(\widetilde{\mathrm{D}}_{C+(t-1) \bar{x}^{s}}\right)$ for any $t \geq 0$.

From now on, we denote $Q_{\diamond} \in L_{\lambda_{\diamond}^{*}} R$ the absolutely continuous part shared by all the solutions of $\left(\overline{\mathrm{P}}_{C}\right)$. Let us introduce

$$
\begin{aligned}
& \mathcal{C}=\left\{Q \in L_{\lambda_{\diamond}^{*}} R ; T Q:=\int_{\mathcal{Z}} \theta \mathrm{d} Q \in C\right\} \\
& \overline{\mathcal{C}}=\left\{\ell \in L_{\lambda_{\diamond}}^{\prime} ; T \ell:=\langle\theta, \ell\rangle \in C\right\}
\end{aligned}
$$

the constraint sets $T^{-1} C \cap L_{\lambda_{\infty}^{*}} R$ and $T^{-1} C$ on which $I$ and $\bar{I}$ are minimized. We have: $\mathcal{C}=\overline{\mathcal{C}} \cap L_{\lambda_{\infty}^{*}} R$ and $I=\bar{I}+\iota_{L_{\lambda_{\delta}^{*} R}}$. Hence, $\inf \left(\overline{\mathrm{P}}_{C}\right) \leq \inf \left(\mathrm{P}_{C}\right)$. Clearly, $\mathcal{C} \cap \operatorname{dom} I \neq \emptyset \Leftrightarrow \inf \left(\mathrm{P}_{C}\right)<\infty \operatorname{implies} \overline{\mathcal{C}} \cap \operatorname{dom} \bar{I} \neq \emptyset \Leftrightarrow$ $\inf \left(\overline{\mathrm{P}}_{C}\right)<\infty \Leftrightarrow C \cap \operatorname{dom} \Gamma^{*} \neq \emptyset$.

Of course, if $\mathcal{C} \cap \operatorname{dom} I \neq \emptyset,\left(\mathrm{P}_{C}\right)$ admits nontrivial minimizing sequences. Theorem 4.4 below gives some details about them.

The present paper is concerned with

$$
\text { minimize } I(Q) \text { subject to } Q \in \mathcal{C} \text {. }
$$

where $\mathcal{C}$ has the form (4.3). But this is not a restriction as explained in the following remarks.

\section{Remark 4.3.}

(1) Taking $T^{*}$ to be the identity on $\mathcal{Y}_{o}=E_{\lambda_{\diamond}}$ or $L_{\lambda_{\diamond}}$ (being careless with a.e. equality, this corresponds to $\theta(z)$ to be the Dirac measure $\left.\delta_{z}\right)$, we see that $\left(\mathrm{A}_{\theta}^{\forall}\right)$ or $\left(\mathrm{A}_{\theta}^{\exists}\right)$ is satisfied respectively. Hence, with $C=\mathcal{C}$, $\left(\mathrm{P}_{C}\right)$ is $\left(\mathcal{P}_{\mathcal{C}}\right)$. Consequently, the specific form with $\theta$ and $C$ adds details to the description of $\mathcal{C}$ without loss of generality. 
(2) With $\theta, \mathcal{Y}_{o}$ and $C$ as in (1), the assumptions on $C=\mathcal{C}$ are:

(a) Under $\left(\mathrm{A}_{\theta}^{\forall}\right),(2.14)$ is equivalent to $\mathcal{C}$ is $\sigma\left(L_{\lambda_{\diamond}^{*}} R, E_{\lambda_{\diamond}}\right)$-closed.

Note that if $\lambda_{\diamond}$ and $\lambda_{\diamond}^{*}$ both satisfy the $\Delta_{2}$-condition, as $\mathcal{C}$ is convex, this is equivalent to $\mathcal{C}$ being $\|\cdot\|_{\lambda_{\diamond}^{*} \text {-closed. }}$

(b) Under $\left(\mathrm{A}_{\theta}^{\exists}\right),(2.18)$ is equivalent to $\mathcal{C}$ is $\sigma\left(L_{\lambda_{\diamond}^{*}} R, L_{\lambda_{\diamond}}\right)$-closed.

Note that if $\lambda_{\diamond}^{*}$ satisfies the $\Delta_{2}$-condition, as $\mathcal{C}$ is convex, this is equivalent to $\mathcal{C}$ being $\|\cdot\|_{\lambda_{\diamond}^{*} \text {-closed. }}$

We denote $\|\cdot\|_{\lambda_{\diamond}^{*}}$-int $(\mathcal{C})$ the interior of $\mathcal{C}$ in $L_{\lambda_{\diamond}^{*}} R$ with respect to the strong topology of $L_{\lambda_{\diamond}^{*}}$.

Theorem 4.4 (minimizing sequences of $\left(\mathrm{P}_{C}\right)$ ). Assume that the hypotheses $(\mathrm{A})$ hold,

$$
\lim _{t \rightarrow \pm \infty} \gamma_{z}^{*}(t) / t=+\infty, \quad \text { for } R \text {-almost every } z \in \mathcal{Z}
$$

and $\mathcal{C}$ which is defined at $(4.3)$ is $\sigma\left(L_{\lambda_{\diamond}^{*}} R, L_{\lambda_{\diamond}}\right)$-closed and satisfies $\mathcal{C} \cap \operatorname{dom} I \neq \emptyset$.

Let us consider the following additional conditions.

(1) (a) There are finitely many moment constraints, i.e. $\mathcal{X}_{o}=\mathbb{R}^{K}$ (see Sect. 3.2);

(b) $\mathcal{C} \cap \operatorname{icordom} I \neq \emptyset$.

(2) $\mathcal{C}$ has a nonempty $\|\cdot\|_{\lambda_{\diamond}^{*} \text {-interior. }}$

Under one of these additional conditions (1) or (2), we have

$$
I\left(Q_{\diamond}\right) \leq \inf \left(\mathrm{P}_{C}\right)=\inf \left(\overline{\mathrm{P}}_{C}\right)
$$

and any minimizing sequence of $\left(\mathrm{P}_{C}\right)$ converges to $Q_{\diamond}$ with respect to $\sigma\left(L_{\lambda_{\diamond}^{*}} R, E_{\lambda_{\diamond}}\right)$ and in variation norm (i.e. strongly in $L_{1} R$ ).

Proof. This proof relies on results which are stated and proved in the remainder of the present section. It is shown at Lemma 4.6 that any minimizing sequence of $\left(\mathrm{P}_{C}\right)$ converges in the sense of the $\sigma\left(L_{\lambda_{\diamond}^{*}} R, E_{\lambda_{\diamond}}\right)$-topology to $Q_{\diamond}$, whenever $\inf \left(\mathrm{P}_{C}\right)=\inf \left(\overline{\mathrm{P}}_{C}\right)$. But this equality holds thanks to Lemma 4.11 and

- under condition (1): Lemma 4.12a;

- under condition (2): Corollary 4.14b.

Let us have a look at the last inequality. For any $\bar{\ell}=\bar{\ell}^{a}+\bar{\ell}^{s}=Q_{\diamond}+\bar{\ell}^{s}$ minimizer of $\left(\overline{\mathrm{P}}_{C}\right)$ and any $\left(Q_{n}\right)_{n \geq 1}$ minimizing sequence of $\left(\mathrm{P}_{C}\right)$, we obtain

$$
\begin{aligned}
\inf _{n} I\left(Q_{n}\right) & =\inf \left(\mathrm{P}_{C}\right)=\inf \left(\overline{\mathrm{P}}_{C}\right)=\bar{I}(\bar{\ell}) \\
& =I\left(Q_{\diamond}\right)+I^{s}\left(\bar{\ell}^{s}\right) \\
& \geq I\left(Q_{\diamond}\right)
\end{aligned}
$$

with a strict inequality if $I^{s}\left(\bar{\ell}^{s}\right)>0$.

Finally, (4.4) implies that $\lambda_{\diamond}$ is finite. As $R$ is assumed to be bounded, we have $L_{\infty} \subset E_{\lambda_{\diamond}}$. But, we also have $L_{\lambda_{\diamond}^{*}} R \subset L_{1} R$ so that the $\sigma\left(L_{\lambda_{\diamond}^{*}} R, L_{\lambda_{\diamond}}\right)$-convergence implies the $\sigma\left(L_{1} R, L_{\infty}\right)$-convergence of any minimizing sequence. The strong convergence in $L_{1} R$ now follows from [2] (Thm. 3.7).

\section{Remark 4.5.}

(1) As regards condition (1), it is not assumed that $\mathcal{C}$ has a nonempty interior.

(2) As regards condition (2):

(i) Any $\sigma\left(L_{\lambda_{\diamond}^{*}} R, L_{\lambda_{\diamond}}\right)$-closed convex set has the form

$$
\mathcal{C}=\bigcap_{u \in U}\left\{\ell \in L_{\lambda_{\diamond}^{*}} R ;\langle u, \ell\rangle \geq a_{u}\right\}
$$

for some $U \subset L_{\lambda_{\diamond}}$. 
(ii) For $\mathcal{C}$ to have a nonempty $\|\cdot\|_{\lambda_{\diamond}^{*}}$-interior, it is enough that $C \cap \mathcal{X}$ has a nonempty interior in $\mathcal{X}$ endowed with the dual norm $|\cdot|_{\lambda_{\diamond}}^{*}$ defined at (4.8). This is a consequence of Lemma 4.9(a) below.

(3) The last quantity $I^{s}\left(\bar{\ell}^{s}\right)=\inf \left(\mathrm{P}_{C}\right)-I\left(Q_{\diamond}\right)$ is precisely the gap of lower $\sigma\left(L_{\lambda_{\diamond}^{*}} R, E_{\lambda_{\diamond}}\right)$-semicontinuity of $I: \lim _{n} Q_{n}=Q_{\diamond}$ and $\inf \left(\mathrm{P}_{C}\right)=\liminf _{n} I\left(Q_{n}\right) \geq I\left(\lim _{n} Q_{n}\right)=I\left(Q_{\diamond}\right)$.

\subsection{A preliminary lemma}

Preliminary results for the proof of Theorem 4.4 are stated below at Lemma 4.6. The assumption $\left(\mathrm{A}_{\theta}^{\exists}\right)$ about the critical constraint is $T^{*} \mathcal{Y}_{o} \subset L_{\lambda_{\diamond}}$.

Lemma 4.6. Assume that the hypotheses (A) and (4.4) hold, $\mathcal{C}$ is $\sigma\left(L_{\lambda_{\diamond}^{*}} R, L_{\lambda_{\diamond}}\right)$-closed, $\inf \left(\mathrm{P}_{C}\right)<\infty$ and

$$
\inf \left(\mathrm{P}_{C}\right)=\inf \left(\overline{\mathrm{P}}_{C}\right)
$$

Then, any minimizing sequence of $\left(\mathrm{P}_{C}\right)$ converges to $Q_{\diamond}$ with respect to $\sigma\left(L_{\lambda_{\diamond}^{*}} R, E_{\lambda_{\diamond}}\right)$.

Proof. Let $\left(Q_{n}\right)_{n \geq 1}$ be a minimizing sequence of $\left(\mathrm{P}_{C}\right)$. Since it is assumed that $\inf \left(\mathrm{P}_{C}\right)=\inf \left(\overline{\mathrm{P}}_{C}\right),\left(Q_{n}\right)_{n \geq 1}$ is also a minimizing sequence of $\left(\overline{\mathrm{P}}_{C}\right)$. By [19] (Lem. 6.2), $\bar{I}$ is $\sigma\left(L_{\lambda_{\diamond}}^{\prime}, L_{\lambda_{\diamond}}\right)$-inf-compact. Hence, we can extract a $\sigma\left(L_{\lambda_{\diamond}}^{\prime}, L_{\lambda_{\diamond}}\right)$-convergent subnet $\left(Q_{\alpha}\right)_{\alpha}$ from $\left(Q_{n}\right)_{n \geq 1}$. Let $\ell_{*} \in \overline{\mathcal{C}}$ denote its limit: we have $\lim _{\alpha} \int_{\mathcal{Z}} u \mathrm{~d} Q_{\alpha}=\left\langle\ell_{*}, u\right\rangle$ for all $u \in L_{\lambda_{\diamond}}$. As $\left\langle\ell_{*}^{s}, u\right\rangle=0$, for all $u \in E_{\lambda_{\diamond}}$ (see Prop. 2.3), we obtain: $\lim _{\alpha} \int_{\mathcal{Z}} u d Q_{\alpha}=\int_{\mathcal{Z}} u \mathrm{~d} \ell_{*}^{a}$ for all $u \in E_{\lambda_{\diamond}}$. This proves that $\left(Q_{\alpha}\right)_{\alpha} \sigma\left(E_{\lambda_{\diamond}}^{\prime}, E_{\lambda_{\diamond}}\right)$-converges to $\ell_{*}^{a}$. As $E_{\lambda_{\diamond}}$ is a separable Banach space $\left(L_{\lambda_{\diamond}}\right.$ is not separable in general), the topology $\sigma\left(E_{\lambda_{\diamond}}^{\prime}, E_{\lambda_{\diamond}}\right)=\sigma\left(L_{\lambda_{\diamond}^{*}} R, E_{\lambda_{\diamond}}\right)$ is metrizable and we can extract a convergent subsequence $\left(\tilde{Q}_{k}\right)_{k \geq 1}$ from the convergent net $\left(Q_{\alpha}\right)_{\alpha}$. Hence, $\left(\tilde{Q}_{k}\right)_{k \geq 1}$ is a subsequence of $\left(Q_{n}\right)_{n \geq 1}$ which $\sigma\left(L_{\lambda_{\diamond}^{*}} R, E_{\lambda_{\diamond}}\right)$-converges to $\ell_{*}^{a}$.

Since $\bar{I}$ is inf-compact, $\ell_{*}$ is a minimizer of $\left(\overline{\mathrm{P}}_{C}\right)$ and by Theorem $4.1 \mathrm{a}$, there is a unique $Q_{\diamond}$ such for any minimizing sequence $\left(Q_{n}\right)_{n \geq 1}, \ell_{*}^{a}=Q_{\diamond}$. Therefore, any convergent subsequence of $\left(Q_{n}\right)_{n \geq 1}$ converges to $Q_{\diamond}$ for $\sigma\left(L_{\lambda_{\diamond}^{*}} R, E_{\lambda_{\diamond}}\right)$. As any subsequence of a minimizing sequence is still a minimizing sequence, we have proved that from any subsequence of $\left(Q_{n}\right)_{n \geq 1}$, we can extract a sub-subsequence which converges to $Q_{\diamond}$. This proves that $\left(Q_{n}\right)_{n \geq 1}$ converges to $Q_{\diamond}$ with respect to $\sigma\left(L_{\lambda_{\diamond}^{*}} R, E_{\lambda_{\diamond}}\right)$.

\subsection{Sufficient conditions for $\inf \left(\mathbf{P}_{C}\right)=\inf \left(\overline{\mathbf{P}}_{C}\right)$}

Our aim now is to obtain sufficient conditions for the identity $\inf \left(\mathrm{P}_{C}\right)=\inf \left(\overline{\mathrm{P}}_{C}\right)$ to hold. Let us rewrite the problems $\left(\mathrm{P}_{C}\right)$ and $\left(\overline{\mathrm{P}}_{C}\right)$ in order to emphasize their differences and analogies. Denote

$$
\begin{aligned}
& \Phi_{L}(u)=I_{\lambda}(u)=\int_{\mathcal{Z}} \lambda(u) \mathrm{d} R, \quad u \in L_{\lambda_{\diamond}} \\
& \Phi_{E}(u)=\Phi_{L}(u)+\iota_{E_{\lambda_{\diamond}}}(u), \quad u \in L_{\lambda_{\diamond}}
\end{aligned}
$$

where $E$ and $L$ refer to $E_{\lambda_{\diamond}}$ and $L_{\lambda_{\diamond}}$. Their convex conjugates are

$$
\begin{array}{ll}
\Phi_{E}^{*}(\ell)=\sup _{u \in E_{\lambda_{\diamond}}}\left\{\langle\ell, u\rangle-I_{\lambda}(u)\right\}, & \ell \in L_{\lambda_{\diamond}^{*}} R \\
\Phi_{L}^{*}(\ell)=\sup _{u \in L_{\lambda_{\diamond}}}\left\{\langle\ell, u\rangle-I_{\lambda}(u)\right\}, \quad \ell \in L_{\lambda_{\diamond}}^{\prime} .
\end{array}
$$

It is shown at [19] (Lem. 6.2) that under the assumption (4.4)

$$
\left\{\begin{array}{l}
I(\ell)=\Phi_{E}^{*}(\ell-m R), \quad \ell \in E_{\lambda_{\diamond}}^{\prime}=L_{\lambda_{\diamond}^{*}} R \\
\bar{I}(\ell)=\Phi_{L}^{*}(\ell-m R), \quad \ell \in L_{\lambda_{\diamond}}^{\prime}=L_{\lambda_{\diamond}^{*}} R \oplus L_{\lambda_{\diamond}}^{s}
\end{array}\right.
$$


Hence, considering the minimization problems

$$
\text { minimize } \Phi_{E}^{*}(\ell) \text { subject to }\langle\theta, \ell\rangle \in C_{o}, \quad \ell \in L_{\lambda_{\diamond}^{*}} R
$$

and

$$
\text { minimize } \Phi_{L}^{*}(\ell) \text { subject to }\langle\theta, \ell\rangle \in C_{o}, \quad \ell \in L_{\lambda_{\diamond}}^{\prime}
$$

with $C_{o}=C-\langle\theta, m R\rangle$, we see that $\ell_{*}$ is a solution of $\left(\mathrm{P}_{C}\right)\left[\right.$ resp. $\left.\left(\overline{\mathrm{P}}_{C}\right)\right]$ if and only if $\ell_{*}-m R$ is a solution of $\left(\mathrm{P}_{E}\right)\left[\right.$ resp. $\left.\left(\mathrm{P}_{L}\right)\right]$. It is enough to prove

$$
\inf \left(\mathrm{P}_{E}\right)=\inf \left(\mathrm{P}_{L}\right)
$$

to get $\inf \left(\mathrm{P}_{C}\right)=\inf \left(\overline{\mathrm{P}}_{C}\right)$.

Basic facts about convex duality. The proof of (4.7) will rely on standard convex duality considerations. Let us recall some related facts, as developed in [26].

Let $A$ and $X$ be two vector spaces, $h: A \rightarrow[-\infty,+\infty]$ a convex function, $T: A \rightarrow X$ a linear operator and $C$ a convex subset of $X$. The primal problem to be considered is the following convex minimization problem

$$
\text { minimize } h(a) \text { subject to } T a \in C, \quad a \in A \text {. }
$$

The primal value-function corresponding to the Fenchel perturbation $F(a, x)=h(a)+\iota_{C}(T a+x), a \in A, x \in X$ is $\varphi(x)=\inf _{a \in A} F(a, x)$, i.e.

$$
\varphi(x)=\inf \{h(a) ; a \in A, T a \in C-x\} \quad x \in X .
$$

Denote $A^{*}$ the algebraic dual space of $A$. The convex conjugate of $h$ with respect to the dual pairing $\left\langle A, A^{*}\right\rangle$ is

$$
h^{*}(\nu)=\sup _{a \in A}\{\langle\nu, a\rangle-h(a)\}, \quad \nu \in A^{*}
$$

Let $Y$ be a vector space in dual pairing with $X$. The adjoint operator $T^{*}: Y \rightarrow A^{*}$ of $T$ is defined for all $y \in Y$ by

$$
\left\langle T^{*} y, a\right\rangle_{A^{*}, A}=\langle y, T a\rangle_{Y, X}, \quad \forall a \in A .
$$

The dual problem associated with $(\mathcal{P})$ is

$$
\text { maximize } \inf _{x \in C}\langle y, x\rangle-h^{*}\left(T^{*} y\right), \quad y \in Y .
$$

Let $U$ be some subspace of $A^{*}$. The dual value-function is

$$
\psi(u)=\sup _{y \in Y}\left\{\inf _{x \in C}\langle y, x\rangle-h^{*}\left(T^{*} y+u\right)\right\}, \quad u \in U
$$

We say that $\langle X, Y\rangle$ is a topological dual pairing if $X$ and $Y$ are topological vector spaces and their topological dual spaces $X^{\prime}$ and $Y^{\prime}$ satisfy $X^{\prime}=Y$ and $Y^{\prime}=X$ up to some isomorphisms.

Theorem 4.7 (criteria for the dual equality). We assume that $A, U, X$ and $Y$ are locally convex Hausdorff topological vector spaces such that $\langle A, U\rangle$ and $\langle X, Y\rangle$ are topological dual pairings. For the dual equality

$$
\inf (\mathcal{P})=\sup (\mathcal{D})
$$


to hold, it is enough that

(1) (a) $h$ is a convex function and $C$ is a convex subset of $X$,

(b) $\varphi$ is lower semicontinuous at $0 \in X$ and

(c) $\sup (\mathcal{D})>-\infty$

or

(2) (a) $h$ is a convex function and $C$ is a closed convex subset of $X$;

(b) $\psi$ is upper semicontinuous at $0 \in U$; and

(c) $\inf (\mathcal{P})<+\infty$.

Remark 4.8. About the space $U$.

(a) As regards criterion (1), the space $U$ is unnecessary.

(b) As regards criterion (2), it is not assumed that $T^{*} Y \subset U$.

The continuity of $T$ and $T^{*}$. The following Lemma 4.9 will be useful for the proof of (4.7). We go back to our usual notation. The Luxemburg norm on $L_{\lambda_{\diamond}}$ is $\|\cdot\|_{\lambda_{\diamond}}$ and its dual norm is

$$
\|\ell\|_{\lambda_{\diamond}}^{*}:=\sup _{u,\|u\|_{\lambda_{\diamond} \leq 1}}|\langle u, \ell\rangle|, \quad \ell \in L_{\lambda_{\diamond}}^{\prime} .
$$

Let us define

$$
|y|_{\lambda_{\diamond}}=\|\langle y, \theta\rangle\|_{\lambda_{\diamond}}, \quad y \in \mathcal{Y}_{o} .
$$

Under the assumption $\left(\mathrm{A}_{\theta}\right),|\cdot|_{\lambda_{\diamond}}$ is a norm on $\mathcal{Y}_{o}$. The dual space of $\left(\mathcal{Y}_{o},|\cdot|_{\lambda_{\diamond}}\right)$ is $\mathcal{X}$ and the corresponding dual norm is

$$
|x|_{\lambda_{\diamond}}^{*}:=\sup _{y,|y|_{\lambda_{\diamond}} \leq 1}|\langle y, x\rangle|, \quad x \in \mathcal{X} .
$$

Lemma 4.9. Let us assume $\left(\mathrm{A}_{\theta}\right)$.

(a) $T: L_{\lambda_{\diamond}}^{\prime} \rightarrow \mathcal{X}$ is $\|\cdot\|_{\lambda_{\diamond}}^{*}-|\cdot|_{\lambda_{\diamond}}^{*}$-continuous,

(b) $T^{*} \mathcal{Y} \subset L_{\lambda_{\diamond}}$ and $T^{*}: \mathcal{Y} \rightarrow L_{\lambda_{\diamond}}$ is $\sigma(\mathcal{Y}, \mathcal{X})-\sigma\left(L_{\lambda_{\diamond}}, L_{\lambda_{\diamond}}^{\prime}\right)$-continuous,

(c) $T: L_{\lambda_{\diamond}}^{\prime} \rightarrow \mathcal{X}$ is $\sigma\left(L_{\lambda_{\diamond}}^{\prime}, L_{\lambda_{\diamond}}\right)-\sigma(\mathcal{X}, \mathcal{Y})$-continuous.

Proof. See [16] (Sect. 4).

Back to our problem. Let us particularize this framework for the problems $\left(\mathrm{P}_{E}\right)$ and $\left(\mathrm{P}_{L}\right)$. Assuming that $m \equiv 0$, we see that $\left(\mathrm{P}_{C}\right)=\left(\mathrm{P}_{E}\right),\left(\overline{\mathrm{P}}_{C}\right)=\left(\mathrm{P}_{L}\right), I=I_{\lambda^{*}}=\Phi_{E}^{*}, \bar{I}=\bar{I}_{\lambda^{*}}=\Phi_{L}^{*}, \gamma=\lambda, C=C_{o}$ and so on. This simplifying requirement will be assumed during the proof without loss of generality, see the proof of [19] (Thm. 4.2.)

Let us first apply the criterion (1) of Theorem 4.7.

Problem $\left(\mathrm{P}_{E}\right)$ is obtained with $A=L_{\lambda_{0}^{*}} R, X=\mathcal{X}, Y=\mathcal{Y}$ equipped with the weak topologies $\sigma(\mathcal{X}, \mathcal{Y})$ and $\sigma(\mathcal{Y}, \mathcal{X})$, and $h=\Phi_{E}^{*}=I$. The corresponding primal value-function is

$$
\varphi_{E}(x)=\inf \left\{I(Q) ; \int_{\mathcal{Z}} \theta \mathrm{d} Q \in C-x, Q \in L_{\lambda_{\triangleright}^{*}} R\right\}, \quad x \in \mathcal{X} .
$$

Under the underlying assumption $\left(\mathrm{A}_{\theta}^{\exists}\right)$, with Lemma $4.9(\mathrm{~b})$ we have:

$$
T^{*} \mathcal{Y} \subset L_{\lambda_{\diamond}} .
$$

Hence, we only need to compute $h^{*}$ on $L_{\lambda_{\diamond}} \subset\left[L_{\lambda_{\diamond}^{*}} R\right]^{*}=A^{*}$. For each $u \in L_{\lambda_{\diamond}}, h^{*}(u)=\sup _{f \in L_{\lambda_{\diamond}^{*}}}\left\{\int_{\mathcal{Z}} u f \mathrm{~d} R-\right.$ $\left.\int_{\mathcal{Z}} \lambda^{*}(f) \mathrm{d} R\right\}$ and it is proved in [25] that

$$
h^{*}(u)=\int_{\mathcal{Z}} \lambda(u) \mathrm{d} R, \quad u \in L_{\lambda_{\diamond}}
$$


Therefore, the dual problem associated to $\left(\mathrm{P}_{E}\right)$ is

$$
\text { maximize } \inf _{x \in C}\langle y, x\rangle-\int_{\mathcal{Z}} \lambda\left(T^{*} y\right) \mathrm{d} R, \quad y \in \mathcal{Y} \text {. }
$$

Let us go on with $\left(\mathrm{P}_{L}\right)$. Take $A=L_{\lambda_{\diamond}}^{\prime}, X=\mathcal{X}, Y=\mathcal{Y}$ equipped with the weak topologies $\sigma(\mathcal{X}, \mathcal{Y})$ and $\sigma(\mathcal{Y}, \mathcal{X})$, and $h=\Phi_{L}^{*}=\bar{I}$. The function $h^{*}$ in restriction to $L_{\lambda_{\diamond}}$ is still given by (4.10) since $u \in L_{\lambda_{\diamond}} \mapsto \int_{\mathcal{Z}} \lambda(u) \mathrm{d} R$ is closed convex (Fatou's lemma). The primal value-function is

$$
\varphi_{L}(x)=\inf \left\{\bar{I}(\ell) ;\langle\theta, \ell\rangle \in C-x, \ell \in L_{\lambda_{\diamond}}^{\prime}\right\}, \quad x \in \mathcal{X}
$$

and the dual problem associated to $\left(\mathrm{P}_{L}\right)$ is

$$
\left(\mathrm{D}_{L}\right)=\left(\mathrm{D}_{E}\right)
$$

Lemma 4.10. Suppose that $T^{*} \mathcal{Y} \subset L_{\lambda_{\diamond}}$ and $C \cap \mathcal{X}$ is $\sigma(\mathcal{X}, \mathcal{Y})$-closed, then $\varphi_{L}$ is $\sigma(\mathcal{X}, \mathcal{Y})$-lower semicontinuous.

Proof. Defining $\tilde{\varphi}(x):=\varphi_{L}(-x)$ and $\bar{J}(x):=\inf \left\{\bar{I}(\ell) ; \ell \in L_{\lambda_{\diamond}}^{\prime}:\langle\theta, \ell\rangle=x\right\}, x \in \mathcal{X}$, we obtain that $\tilde{\varphi}$ is the infconvolution of $\bar{J}$ and the convex indicator of $-C: \iota_{-C}$. That is $\tilde{\varphi}(x)=\left(\bar{J} \square \iota_{-C}\right)(x)=\inf \left\{\bar{J}(y)+\iota_{-C}(z) ; y, z, y+\right.$ $z=x\}$.

As already seen, $\bar{I}$ is $\sigma\left(L_{\lambda_{\diamond}}^{\prime}, L_{\lambda_{\diamond}}\right)$-inf-compact and $T$ is $\sigma\left(L_{\lambda_{\diamond}}^{\prime}, L_{\lambda_{\diamond}}\right)-\sigma(\mathcal{X}, \mathcal{Y})$-continuous, see Lemma 4.9(c). It follows that $\bar{J}$ is $\sigma(\mathcal{X}, \mathcal{Y})$-inf-compact. As $C \cap \mathcal{X}$ is assumed to be $\sigma(\mathcal{X}, \mathcal{Y})$-closed, $\iota_{-} C$ is lower semicontinuous. Finally, being the inf-convolution of an inf-compact function and a lower semicontinuous function, $\tilde{\varphi}$ is lower semicontinuous, and so is $\varphi_{L}$.

As $\bar{I}$ and $C$ are assumed to be convex and $\sup \left(\mathrm{D}_{L}\right) \geq \inf _{x \in C}\langle 0, x\rangle-\int_{\mathcal{Z}} \lambda\left(T^{*} 0\right) \mathrm{d} R=0>-\infty$, this lemma allows us to apply criterion (1) of Theorem 4.7 to obtain

$$
\inf \left(\mathrm{P}_{L}\right)=\sup \left(\mathrm{D}_{L}\right)
$$

Since $\bar{I}$ and $I$ match on $L_{\lambda_{\diamond}^{*}} R$, we have $\inf \left(\mathrm{P}_{L}\right) \leq \inf \left(\mathrm{P}_{E}\right)$. Putting together these considerations gives us

$$
\sup \left(\mathrm{D}_{E}\right)=\sup \left(\mathrm{D}_{L}\right)=\inf \left(\mathrm{P}_{L}\right) \leq \inf \left(\mathrm{P}_{E}\right)
$$

Since the desired equality (4.5) is equivalent to $\inf \left(\mathrm{P}_{L}\right)=\inf \left(\mathrm{P}_{E}\right)$, we have proved

Lemma 4.11. The equality (4.5) holds if and only if we have the dual equality

$$
\inf \left(\mathrm{P}_{E}\right)=\sup \left(\mathrm{D}_{E}\right)
$$

This happens if and only if $\varphi_{E}$ is $\sigma(\mathcal{X}, \mathcal{Y})$-lower semicontinuous at $x=0$.

Let us now give a couple of simple criteria for this property to be realized. 


\section{Lemma 4.12.}

(a) Suppose that there are finitely many constraints (i.e. $\mathcal{X}_{o}$ is finite dimensional) and $\mathcal{C} \cap$ icordom $I \neq \emptyset$, then $\varphi_{E}$ is continuous at 0.

(b) Suppose that $\left(\mathrm{A}_{\theta}^{\forall}\right)$ is satisfied and $C$ is $\sigma(\mathcal{X}, \mathcal{Y})$-closed, then $\varphi_{E}$ is $\sigma(\mathcal{X}, \mathcal{Y})$-lower semicontinuous.

Proof.

- Proof of (a). To get (a), remark that a convex function on a finite dimensional space is lower semicontinuous on the intrinsic core of its effective domain. By Lemma 4.9(c), T is $\sigma\left(L_{\lambda_{\diamond}^{*}} R, L_{\lambda_{\diamond}}\right)$ continuous and the assumption $\mathcal{C} \cap$ icordom $I \neq \emptyset$ implies that 0 belongs to icordom $\varphi_{E}$.

- Proof of (b). It is similar to the proof of Lemma 4.10. The assumption $\left(\mathrm{A}_{\theta}^{\forall}\right)$ insures that $T$ is $\sigma\left(L_{\lambda_{\diamond}^{*}} R, E_{\lambda_{\diamond}}\right)-\sigma(\mathcal{X}, \mathcal{Y})$-continuous.

It follows from Lemmas 4.12b, 4.11, the remark at (4.7) and Lemma 4.6 that under the good constraint assumption $\left(\mathrm{A}_{\theta}^{\forall}\right)$, if $C \cap \operatorname{dom} \Gamma^{*} \neq \emptyset$, then any minimizing sequence of $\left(\mathrm{P}_{C}\right)$ converges with respect to the topology $\sigma\left(L_{\lambda_{\diamond}^{*}} R, E_{\lambda_{\diamond}}\right)$ to the unique solution $\widehat{Q}$ of $\left(\mathrm{P}_{C}\right)$. This is Theorem $2.7 \mathrm{~b}$.

Using criterion (2). Up to now, we only used criterion (1) of Theorem 4.7. In the following lines, we are going to use criterion (2) to prove (4.12) under additional assumptions.

Let us go back to problem $\left(\mathrm{P}_{E}\right)$. It is still assumed without loss of generality that $m=0$ and $\gamma=\lambda$. We introduce a space $U$ and a dual value-function $\psi$ on $U$. The framework of Theorem 4.7 is preserved when taking $X=\mathcal{X}, Y=\mathcal{Y}$ with the weak topologies $\sigma(\mathcal{X}, \mathcal{Y})$ and $\sigma(\mathcal{Y}, \mathcal{X}), h=I$ on $A=L_{\lambda_{*}^{*}} R$ as before and adding the following topological pairing $\langle A, U\rangle$. We endow $A=L_{\lambda_{\triangleright}^{*}} R$ with the topology $\sigma\left(L_{\lambda_{\diamond}^{*}} R, L_{\lambda_{\diamond}}\right)$ and take

$$
U=A^{\prime}=\left(L_{\lambda_{\diamond}^{*}} R\right)^{\prime} \simeq L_{\lambda_{\diamond}}
$$

with the topology $\sigma\left(L_{\lambda_{\diamond}}, L_{\lambda_{\diamond}^{*}}\right)$. By $(4.10): h^{*}=I_{\lambda}$, this leads to the dual value-function

$$
\psi(u)=\sup _{y \in \mathcal{Y}}\left\{\inf _{x \in C}\langle y, x\rangle-I_{\lambda}\left(T^{*} y+u\right)\right\}, \quad u \in L_{\lambda_{\diamond}} .
$$

To apply criterion (2), let us establish the following

Lemma 4.13. For $\psi$ to be $\sigma\left(L_{\lambda_{\diamond}}, L_{\lambda_{\diamond}^{*}}\right)$-upper semicontinuous, it is enough that

(a) $T^{*} \mathcal{Y}$ is a $\sigma\left(L_{\lambda_{\diamond}}, L_{\lambda_{\diamond}^{*}}\right)$-closed subspace of $L_{\lambda_{\diamond}}$ and

(b) the interior of $\mathcal{C}$ in $L_{\lambda_{\diamond}^{*}} R$ with respect to $\|\cdot\|_{\lambda_{\diamond}^{*}}$ is nonempty.

Proof. During this proof, unless specified the topology on $L_{\lambda_{\diamond}}$ is $\sigma\left(L_{\lambda_{\diamond}}, L_{\lambda_{\diamond}^{*}}\right)$. For all $u \in L_{\lambda_{\diamond}}$,

$$
\begin{aligned}
-\psi(u) & =\inf _{y \in \mathcal{Y}}\left\{\sup \left\{\left\langle-T^{*} y, \ell\right\rangle ; \ell \in L_{\lambda_{\diamond}^{*}} R: T \ell \in C\right\}+I_{\lambda}\left(T^{*} y+u\right)\right\} \\
& =\inf _{v \in V}\left\{\sup \{\langle-v, \ell\rangle ; \ell \in \mathcal{C}\}+I_{\lambda}(v+u)\right\} \\
& =I_{\lambda} \square G(u)
\end{aligned}
$$

where $V=T^{*} \mathcal{Y}$ and $I_{\lambda} \square G(u)=\inf _{v \in L_{\lambda_{\diamond}}}\left\{G(v)+I_{\lambda}(u-v)\right\}$ is the inf-convolution of $I_{\lambda}$ and $G(u)=\iota_{\mathcal{C}}^{*}(u)+$ $\iota_{V}(u), u \in L_{\lambda_{\diamond}}$. Let us show that under the assumption (a),

$$
G=\iota_{\mathcal{C}}^{*}
$$

As $V$ is assumed to be closed, we have $\iota_{V}=\iota_{V^{\perp}}^{*}$ with $V^{\perp}=\left\{k \in L_{\lambda_{\diamond}^{*}} R ;\langle v, k\rangle=0, \forall v \in V\right\}$. This gives for each $u \in L_{\lambda_{\diamond}^{*}}^{\prime}, G(u)=\iota_{\mathcal{C}}^{*}(u)+\iota_{V^{\perp}}^{*}(u)=\sup _{\ell \in \mathcal{C}}\langle u, \ell\rangle+\sup _{k \in V^{\perp}}\langle u, k\rangle=\sup \left\{\langle u, \ell+k\rangle ; \ell \in \mathcal{C}, k \in V^{\perp}\right\}=\iota_{\mathcal{C}+V^{\perp}}^{*}(u)=$ $\iota_{\mathcal{C}}^{*}(u)$, where the last equality holds since $\mathcal{C}+V^{\perp}=\mathcal{C}$, note that $\operatorname{ker} T=V^{\perp}$. 
As convex conjugates, $\iota_{\mathcal{C}}^{*}$ and $I_{\lambda}=h^{*}$ are closed convex functions.

Since for all $u, v \in L_{\lambda_{\diamond}}$,

$$
\begin{aligned}
\iota_{\mathcal{C}}^{*}(v)+I_{\lambda}(u-v) & =\iota_{\mathcal{C}-k}^{*}(v)-\langle v, k\rangle+I_{\lambda}(u-v) \\
& =\iota_{\mathcal{C}-k}^{*}(v)+\langle u-v, k\rangle+I_{\lambda}(u-v)-\langle u, k\rangle,
\end{aligned}
$$

we have

$$
-\psi+\langle\cdot, k\rangle=\iota_{\mathcal{C}-k}^{*} \square\left(I_{\lambda}-\langle\cdot, k\rangle\right) .
$$

But, by the assumption (b) there exists some $k$ in $L_{\lambda_{\triangleright}^{*}} R$ such that $0 \in \operatorname{int}(\mathcal{C}-k)$. It follows that $\iota_{\mathcal{C}-k}^{*}$ is inf-compact. Finally, $-\psi+\langle\cdot, k\rangle$ is lower semicontinuous, being the inf-convolution of a lower semicontinuous and an inf-compact functions.

Corollary 4.14. Assume that the hypotheses (A) and (4.4) hold.

(a) Assume in addition that $C$ is $\sigma(\mathcal{X}, \mathcal{Y})$-closed convex, $T^{*} \mathcal{Y}$ is a $\sigma\left(L_{\lambda_{\diamond}}, L_{\lambda_{\diamond}^{*}}\right)$-closed subspace of $L_{\lambda_{\diamond}}, \mathcal{C}$ has a

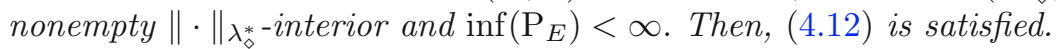

(b) In particular, if $\mathcal{C}$ is $\sigma\left(L_{\lambda_{\diamond}^{*}} R, L_{\lambda_{\diamond}}\right)$-closed convex set with a nonempty $\|\cdot\|_{\lambda_{\diamond}^{*}}$-interior and $\inf \left(\mathrm{P}_{E}\right)<\infty$, then (4.12) is satisfied.

Proof.

- Proof of (a). Apply the criterion (2) of Theorem 4.7 with Lemma 4.13.

- Proof of (b). This is (a) with $\mathcal{Y}=L_{\lambda_{\diamond}}$ and $T^{*}=\mathrm{Id}$, taking advantage of Remarks 4.3.

\section{Entropic PROJECTIONS}

The results of the preceding sections are translated in terms of entropic projections. We consider the problem $\left(\mathcal{P}_{\mathcal{C}}\right)$ where $\mathcal{C}$ is a convex subset of $M_{\mathcal{Z}}$.

\subsection{Generalized entropic projections.}

Let us start recalling an interesting result of Csiszár.

Definition 5.1. Let $Q$ and $\left(Q_{n}\right)_{n \geq 1}$ in $M_{\mathcal{Z}}$ be absolutely continuous with respect to $R$.

(1) One says that $\left(Q_{n}\right)_{n \geq 1}$ converges in $R$-measure to $Q$ if $\frac{\mathrm{d} Q_{n}}{\mathrm{~d} R}$ converges in $R$-measure to $\frac{\mathrm{d} Q}{\mathrm{~d} R}$.

(2) One says that $\left(Q_{n}\right)_{n \geq 1}$ converges $\sigma\left(L_{\lambda_{\diamond}^{*}} R, E_{\lambda_{\diamond}}\right)$ to $Q$ in $L_{\lambda_{\diamond}^{*}} R$ if $\frac{\mathrm{d} Q_{n}}{\mathrm{~d} R} \sigma\left(L_{\lambda_{\diamond}^{*}} R, E_{\lambda_{\diamond}}\right)$-converges to $\frac{\mathrm{d} Q}{\mathrm{~d} R}$.

(3) One says that $\left(Q_{n}\right)_{n \geq 1}$ converges in variation to $Q$ if $\frac{\mathrm{d} Q_{n}}{\mathrm{~d} R}$ converges to $\frac{\mathrm{d} Q}{\mathrm{~d} R}$ in $L_{1}$.

Definition 5.2 (generalized entropic projection [5], Csiszár). Suppose that $\mathcal{C} \cap \operatorname{dom} I \neq \emptyset$ and that any minimizing sequence of the problem $\left(\mathcal{P}_{\mathcal{C}}\right)$ converges in variation to some $Q_{*} \in M_{\mathcal{Z}}$. This $Q_{*}$ is called the generalized $I$-projection of $m R$ on $\mathcal{C}$ with respect to $I$. It may not belong to $\mathcal{C}$. In case $Q_{*}$ is in $\mathcal{C}$, it is called the $I$-projection of $m R$ on $\mathcal{C}$.

Theorem 5.3 (Csiszár $[6])$. Suppose that $\left(\mathrm{A}_{R}\right)$ and $\left(\mathrm{A}_{\gamma^{*}}\right)$ are satisfied. Then, $m R$ has a generalized I-projection on any convex subset $\mathcal{C}$ of $M_{\mathcal{Z}}$ such that $\mathcal{C} \cap \operatorname{dom} I \neq \emptyset$.

In [6] $\gamma^{*}$ doesn't depend on the variable $z$, but the proof remains unchanged with a $z$-dependence.

Csiszár's proof of Theorem 5.3 is based on a parallelogram identity which allows to show that any minimizing sequence is a Cauchy sequence. This result is general but it doesn't tell much about the nature of $Q_{*}$. Let us give some details on the generalized entropic projections in specific situations.

As a direct consequence of Theorem 2.7, if the constraints are good, the generalized entropic projection is the entropic projection. 
Proposition 5.4. Suppose that (A), $\left(\mathrm{A}_{\theta}^{\forall}\right)$ and (4.4) hold, $C$ is convex, $\mathcal{C}$ given at (4.3) is $\sigma\left(L_{\lambda_{\diamond}^{*}} R, E_{\lambda_{\diamond}}\right)$-closed and $\mathcal{C} \cap \operatorname{dom} I \neq \emptyset$. Then, the $I$-projection $Q_{*}$ exists and is equal to

$$
Q_{*}=\widehat{Q} \in \mathcal{C}
$$

where $\widehat{Q}$ is the minimizer of $\left(\mathrm{P}_{C}\right)$ which is described at Theorem 2.7.

Moreover, any minimizing sequence $\sigma\left(L_{\lambda_{\diamond}^{*}} R, L_{\lambda_{\diamond}}\right)$-converges to $Q_{*}$.

Proof. This is an easy corollary of Theorem 2.7.

As a direct corollary of Proposition 5.4 and Remarks 4.3, we obtain the following result which is essentially [6] (Thm. 3(iii)).

Corollary 5.5. Suppose that (A) and (4.4) hold. Let $\mathcal{C}$ be any $\sigma\left(L_{\lambda_{\odot}^{*}} R, E_{\lambda_{\diamond}}\right)$-closed convex set such that $\mathcal{C} \cap \operatorname{dom} I \neq \emptyset$. Then, the $I$-projection of $m R$ on $\mathcal{C}$ exists.

Theorem 5.6. Suppose that (A) and (4.4) hold, $\mathcal{C}$ given at (4.3) is $\sigma\left(L_{\lambda_{\diamond}^{*}} R, L_{\lambda_{\diamond}}\right)$-closed and $\mathcal{C} \cap \operatorname{dom} I \neq \emptyset$. Let us consider the additional conditions:

(1) (a) There are finitely many moment constraints, i.e. $\mathcal{X}_{o}=\mathbb{R}^{K}$ (see Sect. 3.2)

(b) $\mathcal{C} \cap$ icordom $I \neq \emptyset$;

(2) $\mathcal{C}$ is a $\sigma\left(L_{\lambda_{\diamond}^{*}} R, L_{\lambda_{\diamond}}\right)$-closed convex set with a nonempty $\|\cdot\|_{\lambda_{\diamond}^{*} \text {-interior. }}$

Then, under one of the conditions (1) or (2), the generalized I-projection $Q_{*}$ of $m R$ on $\mathcal{C}$ is

$$
Q_{*}=Q_{\diamond}
$$

the absolutely continuous component described at (4.1) and $I\left(Q_{*}\right) \leq \inf _{\mathcal{C}} I$.

Proof. This is a direct consequence of Theorem 4.4.

\subsection{The special case of relative entropy}

The relative entropy $I(P \mid R)$ and its extension $\bar{I}(\ell \mid R)$ are described at Section 3. The minimization problem is

$$
\text { minimize } I(P \mid R) \text { subject to } \int_{\mathcal{Z}} \theta \mathrm{d} P \in C, \quad P \in P_{\mathcal{Z}}
$$

and its extension is

$$
\text { minimize } \bar{I}(\ell \mid R) \text { subject to }\langle\theta, \ell\rangle \in C, \quad \ell \in O_{\exp } .
$$

Recall that $O_{\exp }$ is defined at (3.4). We introduce the Cramér transform of the image law of $R$ by $\theta$ on $\mathcal{X}_{o}$ :

$$
\Xi(x)=\sup _{y \in \mathcal{Y}_{o}}\left\{\langle y, x\rangle-\log \int_{\mathcal{Z}} \mathrm{e}^{\langle y, \theta\rangle} \mathrm{d} R\right\} \in[0, \infty], \quad x \in \mathcal{X}_{o} .
$$

Proposition 5.7 (relative entropy subject to good constraints). Let us assume that $\theta$ satisfies the "good constraint" assumption

$$
\forall y \in \mathcal{Y}_{o}, \int_{\mathcal{Z}} \mathrm{e}^{\langle y, \theta(z)\rangle} R(\mathrm{~d} z)<\infty
$$

and that $C \cap \mathcal{X}$ is a $\sigma(\mathcal{X}, \mathcal{Y})$-closed convex subset of $\mathcal{X}$.

(a) The following dual equality holds:

$$
\inf \left\{I(P \mid R) ;\langle\theta, P\rangle \in C, P \in P_{\mathcal{Z}}\right\}=\sup _{y \in \mathcal{Y}_{o}}\left\{\inf _{x \in C}\langle y, x\rangle-\log \int_{\mathcal{Z}} \mathrm{e}^{\langle y, \theta\rangle} \mathrm{d} R\right\} \in[0, \infty] .
$$


(b) Suppose that in addition $C \cap \operatorname{dom} \Xi \neq \emptyset$. Then, the minimization problem (5.1) has a unique solution $\widehat{P}$ in $P_{\mathcal{Z}}, \widehat{P}$ is the entropic projection of $R$ on $\mathcal{C}=\left\{P \in P_{\mathcal{Z}}, \int_{\mathcal{Z}} \theta \mathrm{d} P \in C\right\}$.

(c) Suppose that in addition, $C \cap$ icordom $\Xi \neq \emptyset$, then there exists some linear form $\tilde{\omega}$ on $\mathcal{X}_{o}$ such that $\langle\tilde{\omega}, \theta\rangle$ is measurable and

$$
\left\{\begin{array}{l}
\hat{x}:=\int_{\mathcal{Z}} \theta \mathrm{d} \widehat{P} \in C \cap \operatorname{dom} \Xi \\
\langle\tilde{\omega}, \hat{x}\rangle \leq\langle\tilde{\omega}, x\rangle, \forall x \in C \cap \operatorname{dom} \Xi \\
\widehat{P}(d z)=\exp \left(\langle\tilde{\omega}, \theta(z)\rangle-\log \int_{\mathcal{Z}} e^{\langle\tilde{\omega}, \theta\rangle} \mathrm{d} R\right) R(\mathrm{~d} z) .
\end{array}\right.
$$

In this situation, $\hat{x}$ minimizes $\Xi$ on $C, I(\widehat{P} \mid R)=\Xi(\hat{x})$ and

$$
\hat{x}=\int_{\mathcal{Z}} \theta(z) \exp \left(\langle\tilde{\omega}, \theta(z)\rangle-\log \int_{\mathcal{Z}} \mathrm{e}^{\langle\tilde{\omega}, \theta\rangle} \mathrm{d} R\right) R(\mathrm{~d} z)
$$

in the weak sense. Moreover, $\tilde{\omega}$ satisfies (5.5) if and only if it is the solution to

$$
\operatorname{maximize} \inf _{x \in C \cap \mathcal{X}}\langle\omega, x\rangle-\log \int_{\mathcal{Z}} \mathrm{e}^{\langle\omega, \theta\rangle} \mathrm{d} R, \quad \omega \in \tilde{\mathcal{Y}}
$$

where $\tilde{\mathcal{Y}}$ is the cone of all measurable functions $u$ such that $u_{-}$is in the $\|\cdot\|_{1}$-closure of $\left\langle\mathcal{Y}_{o}, \theta\right\rangle_{-}$and $u_{+}$is in the $\sigma\left(L_{\tau}, L_{\tau^{*}}\right)$-closure of $\left\langle\mathcal{Y}_{o}, \theta\right\rangle_{+}$.

Proposition 5.8 (relative entropy subject to critical constraints). Let us assume that $\theta$ satisfies the "critical constraint" assumption

$$
\forall y \in \mathcal{Y}_{o}, \exists \alpha>0, \int_{\mathcal{Z}} \mathrm{e}^{\alpha|\langle y, \theta(z)\rangle|} R(\mathrm{~d} z)<\infty
$$

and that $C \cap \mathcal{X}$ is a $\sigma(\mathcal{X}, \mathcal{Y})$-closed convex subset of $\mathcal{X}$

(a) The following dual equality holds:

$$
\inf \left\{\bar{I}(\ell \mid R) ;\langle\theta, \ell\rangle \in C, \ell \in O_{\exp }\right\}=\sup _{y \in \mathcal{Y}_{o}}\left\{\inf _{x \in C}\langle y, x\rangle-\log \int_{\mathcal{Z}} \mathrm{e}^{\langle y, \theta\rangle} \mathrm{d} R\right\} \in[0, \infty]
$$

(b) Suppose that in addition $C \cap \operatorname{dom} \Xi \neq \emptyset$. Then, the minimization problem (5.2) is attained in $O_{\exp }$ : the set of minimizers is nonempty, convex and $\sigma\left(L_{\tau}^{\prime}, L_{\tau}\right)$-compact. Moreover, all the minimizers share the same unique absolutely continuous part $P_{\diamond} \in P_{\mathcal{Z}} \cap L_{\tau^{*}} R$ which is the generalized entropic projection of $R$ on $\mathcal{C}$.

(c) Suppose that in addition, one of the following conditions

(1) $\mathcal{X}_{o}=\mathbb{R}^{K}$ and $\mathcal{C} \cap$ icordom $I \neq \emptyset$ or

(2) $\|\cdot\|_{\tau^{*}}-\operatorname{int}(\mathcal{C}) \neq \emptyset$.

is satisfied. Then, there exists a linear form $\tilde{\omega}$ on $\mathcal{X}_{o}$ such that $\langle\tilde{\omega}, \theta\rangle$ is measurable, $\int_{\mathcal{Z}} \mathrm{e}^{\langle\tilde{\omega}, \theta\rangle} \mathrm{d} R<\infty$ and

$$
P_{\diamond}(d z)=\exp \left(\langle\tilde{\omega}, \theta(z)\rangle-\log \int_{\mathcal{Z}} \mathrm{e}^{\langle\tilde{\omega}, \theta\rangle} \mathrm{d} R\right) R(\mathrm{~d} z)
$$

Moreover, $\tilde{\omega}$ is the solution to (5.7).

In Proposition 5.7, $\hat{x}$ is the dominating point in the sense of Ney (see Def. 6.2) of $C$ with respect to $\Xi$. The representation of $\hat{x}$ has already been obtained for $C$ with a nonempty topological interior by Ney [23] and Einmahl and Kuelbs [10], respectively in $\mathbb{R}^{d}$ and in a Banach space setting. The representation of the generalized projection $P_{\diamond}$ is obtained with a very different proof by Csiszár [4] and ([5], Thm. 3). Proposition 5.8 also extends corresponding results of Kuelbs [14] which are obtained in a Banach space setting.

Proof of Propositions 5.7 and 5.8. They are direct consequences of Theorems 2.7, 2.8, Proposition 5.4, Theorem 5.6 and Lemma 5.9 below. 
The following lemma allows to apply the results of the present paper with $\gamma(s)=\mathrm{e}^{s}-1$ and the extended constraint $\langle(\mathbf{1}, \theta), \ell\rangle \in\{1\} \times C$ : the first component of the constraint insures the unit mass $\langle\mathbf{1}, \ell\rangle=1$, to obtain results in terms of log-Laplace transform.

Lemma 5.9. For all $x \in \mathcal{X}_{o}$,

$$
\sup _{y \in \mathcal{Y}_{o}}\left\{\langle y, x\rangle-\log \int_{\mathcal{Z}} \mathrm{e}^{\langle y, \theta\rangle} \mathrm{d} R\right\}=\sup _{\tilde{y} \in \mathbb{R} \times \mathcal{Y}_{o}}\left\{\langle\tilde{y},(1, x)\rangle-\int_{\mathcal{Z}}\left(\mathrm{e}^{\langle\tilde{y},(1, \theta)\rangle}-1\right) \mathrm{d} R\right\} \in(-\infty,+\infty] .
$$

Proof. Using the identity: $-\log b=\sup _{a}\left\{a+1-b \mathrm{e}^{a}\right\}$, we get:

$$
\begin{aligned}
\sup _{y \in \mathcal{Y}_{o}}\left\{\langle y, x\rangle-\log \int_{\mathcal{Z}} \mathrm{e}^{\langle y, \theta\rangle} \mathrm{d} R\right\} & =\sup _{a \in \mathbb{R}, y \in \mathcal{Y}_{o}}\left\{\langle y, x\rangle+a+1-\mathrm{e}^{a} \int_{\mathcal{Z}} \mathrm{e}^{\langle y, \theta\rangle} \mathrm{d} R\right\} \\
& =\sup _{a \in \mathbb{R}, y \in \mathcal{Y}_{o}}\left\{\langle(a, y),(1, x)\rangle-\int_{\mathcal{Z}} \mathrm{e}^{\langle y, \theta\rangle+a} \mathrm{~d} R+1\right\} \\
& =\sup _{\tilde{y} \in \mathbb{R} \times \mathcal{Y}_{o}}\left\{\langle\tilde{y},(1, x)\rangle-\int_{\mathcal{Z}}\left(\mathrm{e}^{\langle\tilde{y},(1, \theta)\rangle}-1\right) \mathrm{d} R\right\}
\end{aligned}
$$

which is the desired result.

Formula (5.3) states that $\Xi=\Lambda^{*}$ where

$$
\Lambda(y)=\log \int_{\mathcal{Z}} \mathrm{e}^{\langle y, \theta\rangle} \mathrm{d} R, \quad y \in \mathcal{Y}_{o}
$$

is the log-Laplace transform of the image of $R$ by the mapping $\theta$. On the other hand, Lemma 5.9 states that $\Lambda^{*}(x)=\Gamma^{*}(1, x)$. Hence, when working with the relative entropy, one can switch from $\Gamma$ to $\Lambda$.

Example 5.10. Csiszár's example. Comparing Proposition 5.4 with Theorem 5.6, one may wonder if the $\sigma\left(L_{\lambda_{\diamond}^{*}} R, E_{\lambda_{\diamond}}\right)$-closedness of the convex set $\mathcal{C}$ is critical for the existence of an entropic projection. The answer is affirmative. In [5] (ex. 3.2), Csiszár gives an interesting example where the generalized entropic projection can be explicitly computed in a situation where $\left(\mathrm{P}_{C}\right)$ is not attained, see also [9] (exercise 7.3.11). This example is the following one.

Take the probability measure on $\mathcal{Z}=[0, \infty)$ defined by $R(\mathrm{~d} z)=\frac{1}{a_{0}} \frac{\mathrm{e}^{-z}}{1+z^{3}} \mathrm{~d} z$ where $a_{0}$ is the normalizing constant, $I$ the relative entropy with respect to $R, \theta: z \in[0, \infty) \mapsto z \in \mathcal{X}=\mathbb{R}$ and $C=[c, \infty)$. This gives $\mathcal{C}=\left\{Q \in L_{\tau^{*}} R ; \int_{[0, \infty)} z Q(\mathrm{~d} z) \geq c, Q([0, \infty))=1\right\}$

From the point of view of the present article, the main point is that $\theta$ is in $L_{\tau}(R)$ but not in $E_{\tau}(R)$. Indeed, the log-Laplace transform is

$$
\Lambda(y)=\log \int_{[0, \infty)} \mathrm{e}^{(y-1) z} \frac{\mathrm{d} z}{a_{0}\left(1+z^{3}\right)}, \quad y \in \mathbb{R}
$$

whose effective domain is $(-\infty, 1]$ which admits 0 as an interior point but is not the whole line. A graphic representation of $\Lambda$ is drawn at Figure 3 below, see example 6.9.

By Theorem 5.8, the generalized projection of $R$ on $\mathcal{C}$ exists and is equal to $P_{y}=\frac{1}{a_{y}} \frac{\mathrm{e}^{(y-1) z}}{1+z^{3}} \mathrm{~d} z$ for some real $y \leq 1$, where $a_{y}=a_{0} \mathrm{e}^{\Lambda(y)}$ is the normalizing constant. If it belongs to $\mathcal{C}$, then $\Lambda^{\prime}(y)=\int_{\mathcal{Z}} z P_{y}(\mathrm{~d} z) \geq c$. But $\Lambda$ is convex so that $\Lambda^{\prime}$ is increasing and $\sup _{y \leq 1} \int_{\mathcal{Z}} z P_{y}(\mathrm{~d} z)=\int_{\mathcal{Z}} z P_{1}(\mathrm{~d} z)=\frac{1}{a_{1}} \int_{\mathcal{Z}} \frac{z}{1+z^{3}} \mathrm{~d} z:=x_{*}<\infty$. Therefore, for any $c>x_{*}$, there are no entropic projection but only a generalized one which is $P_{1}(\mathrm{~d} z)=\frac{\mathrm{d} z}{a_{1}\left(1+z^{3}\right)}$.

A detailed analysis of this example in terms of singular component is done by Léonard and Najim [20] (Prop. 3.9). This example corresponds to a $\sigma\left(L_{\lambda_{\diamond}^{*}} R, L_{\lambda_{\diamond}}\right)$-closed convex set $\mathcal{C}$ such that no entropic projection exists but only a generalized one.

More details about this example are given below at examples 6.9 and 7.2. 


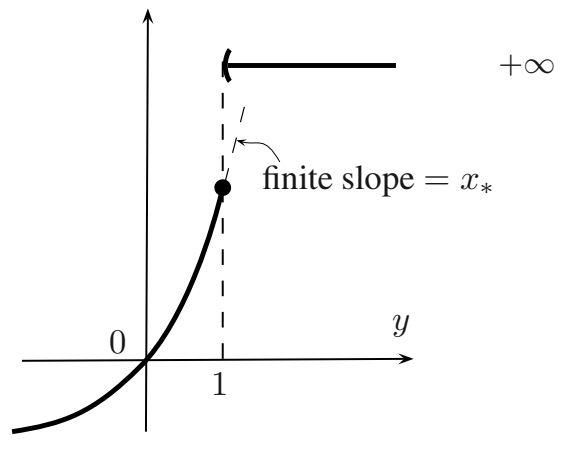

Graph of $\Lambda$

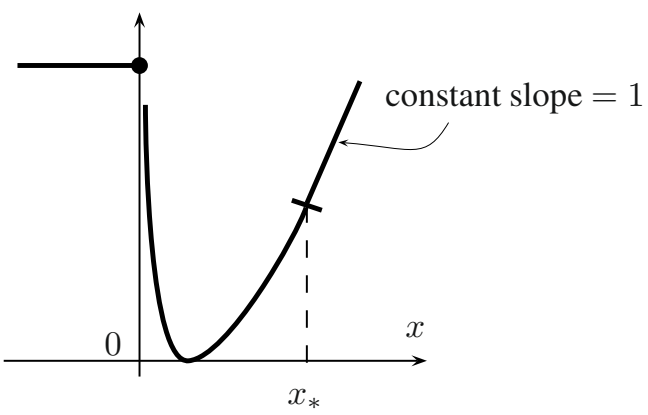

Graph of $\Xi=\Lambda^{*}$

Figure 3. The case: $R(\mathrm{~d} z)=\frac{1}{a_{0}} \frac{\mathrm{e}^{-z}}{1+z^{3}} \mathrm{~d} z$.

\section{Dominating Points}

Following Ney [22,23], let us introduce the following definition. A point $\hat{x} \in \mathcal{X}_{o}$ sharing the properties $(2.15)(\mathrm{a}, \mathrm{b})$ and $(2.16)$ of Theorem 2.7 is called a dominating point.

Definition 6.1 (dominating point). Let $C \subset \mathcal{X}_{o}$ be a convex set such that $C \cap \mathcal{X}$ is $\sigma(\mathcal{X}, \mathcal{Y})$-closed. The point $\hat{x} \in \mathcal{X}_{o}$ is called a $\Gamma^{*}$-dominating point if

(a) $\hat{x} \in C \cap \operatorname{dom} \Gamma^{*}$,

(b) there exists some linear form $\tilde{\omega}$ on $\mathcal{X}_{o}$ such that $\langle\tilde{\omega}, \hat{x}\rangle \leq\langle\tilde{\omega}, x\rangle$ for all $x \in C \cap \operatorname{dom} \Gamma^{*}$,

(c) $\langle\tilde{\omega}, \theta(\cdot)\rangle$ is measurable and $\hat{x}=\int_{\mathcal{Z}} \theta(z) \gamma^{\prime}(z,\langle\tilde{\omega}, \theta(z)\rangle) R(\mathrm{~d} z)$.

In the special case where $\Gamma^{*}$ is replaced by the Cramér transform $\Xi$ defined at (5.3), taking Lemma 5.9 into account, this definition becomes the following one.

Definition 6.2. Let $C \subset \mathcal{X}_{o}$ be a convex set such that $C \cap \mathcal{X}$ is $\sigma(\mathcal{X}, \mathcal{Y})$-closed. The point $\hat{x} \in \mathcal{X}_{o}$ is called a $\Xi$-dominating point if

(a) $\hat{x} \in C \cap \operatorname{dom} \Xi$;

(b) there exists some linear form $\tilde{\omega}$ on $\mathcal{X}_{o}$ such that $\langle\tilde{\omega}, \hat{x}\rangle \leq\langle\tilde{\omega}, x\rangle$, for all $x \in C \cap \operatorname{dom} \Xi$ and

(c) $\langle\tilde{\omega}, \theta(\cdot)\rangle$ is measurable and $\hat{x}=\int_{\mathcal{Z}} \theta(z) \frac{\exp (\langle\tilde{\omega}, \theta(z)\rangle)}{Z(\tilde{\omega})} R(\mathrm{~d} z)$ where $Z(\tilde{\omega})$ is the unit mass normalizing constant.

Note that this definition is slightly different from the ones proposed by Ney [23] and Einmahl and Kuelbs [10] since $C$ is neither supposed to be an open set nor to have a non-empty interior and $\hat{x}$ is not assumed to be a boundary point of $C$. The above integral representation (c) is (5.6).

We are going to investigate some relations between dominating points and entropic projections. In the case where the constraint is good, Theorem 2.7 and Proposition 5.4 state that the generalized entropic projection is the entropic projection $Q_{*}=\widehat{Q}$, the minimizer $\bar{x}$ is the dominating point of $C$, it is related to $Q_{*}$ by the identity:

$$
\bar{x}=\left\langle\theta, Q_{*}\right\rangle .
$$

We now look at the situation where the constraint is critical. As remarked at example 5.10, the above equality may fail. A necessary and sufficient condition (in terms of the function $\Gamma^{*}$ ) for $\bar{x}$ to satisfy (6.1) is obtained at Theorem 6.7. 
Recall that the extended entropy $\bar{I}$ is given by (2.9): $\bar{I}(\ell)=I\left(\ell^{a}\right)+I^{s}\left(\ell^{s}\right), \ell=\ell^{a}+\ell^{s} \in L_{\lambda_{\diamond}}^{\prime}$ and define for all $x \in \mathcal{X}_{o}$,

$$
\begin{aligned}
\bar{J}(x) & :=\inf \left\{\bar{I}(\ell) ; \ell \in L_{\lambda_{\diamond}}^{\prime},\langle\theta, \ell\rangle=x\right\} \\
J(x) & :=\inf \left\{I(\ell) ; \ell \in L_{\lambda_{\diamond}^{*}} R,\langle\theta, \ell\rangle=x\right\} \\
J^{s}(x) & :=\inf \left\{I^{s}(\ell) ; \ell \in L_{\lambda_{\diamond}}^{s},\langle\theta, \ell\rangle=x\right\} .
\end{aligned}
$$

Because of the decomposition $L_{\lambda_{\diamond}}^{\prime} \simeq L_{\lambda_{\diamond}^{*}} R \oplus L_{\lambda_{\diamond}}^{s}$, we obtain for all $x \in \mathcal{X}_{o}$,

$$
\begin{aligned}
\bar{J}(x) & =\inf \left\{I\left(\ell_{1}\right)+I^{s}\left(\ell_{2}\right) ; \ell_{1} \in L_{\lambda_{\diamond}^{*}} R, \ell_{2} \in L_{\lambda_{\diamond}}^{s},\left\langle\theta, \ell_{1}+\ell_{2}\right\rangle=x\right\} \\
& =\inf \left\{J\left(x_{1}\right)+J^{s}\left(x_{2}\right) ; x_{1}, x_{2} \in \mathcal{X}_{o}, x_{1}+x_{2}=x\right\} \\
& =J \square J^{s}(x)
\end{aligned}
$$

where $J \square J^{s}$ is the inf-convolution of $J$ and $J^{s}$. By Theorem 2.8a, $\bar{J}=\Gamma^{*}$ and if $\bar{J}(x)<\infty$, there exists $\ell_{x} \in L_{\lambda_{\diamond}}^{\prime}$ such that $\left\langle\theta, \ell_{x}\right\rangle=x$ and $\bar{J}(x)=\bar{I}\left(\ell_{x}\right)$. Let us define

$$
x^{a}:=\left\langle\theta, \ell_{x}^{a}\right\rangle \quad \text { and } \quad x^{s}:=\left\langle\theta, \ell_{x}^{s}\right\rangle .
$$

These definitions make sense since $\ell_{x}^{a}$ is the unique (common) absolutely continuous part of the minimizers of $\bar{I}$ on the closed convex set $\left\{\ell \in L_{\lambda_{\diamond}}^{\prime} ;\langle\theta, \ell\rangle=x\right\}$, see Theorem 4.1a. Of course, we have

$$
x=x^{a}+x^{s}
$$

and as $\bar{J}(x)=\bar{I}\left(\ell_{x}\right)=I\left(\ell_{x}^{a}\right)+I^{s}\left(\ell_{x}^{s}\right) \geq J\left(x^{a}\right)+J^{s}\left(x^{s}\right) \geq J \square J^{s}(x)=\bar{J}(x)$, we get the following result.

Proposition 6.3. For all $x \in \operatorname{dom} \bar{J}$, we have:

$$
\bar{J}(x)=J\left(x^{a}\right)+J^{s}\left(x^{s}\right), J\left(x^{a}\right)=I\left(\ell_{x}^{a}\right) \text { and } J^{s}\left(x^{s}\right)=I^{s}\left(\ell_{x}^{s}\right) .
$$

Now, let us have a look at the dual equalities. The recession function of $\Gamma^{*}$ is defined for all $x$ by

$$
\widetilde{\Gamma^{*}}(x):=\lim _{t \rightarrow+\infty} \Gamma^{*}(t x) / t \in(-\infty,+\infty] .
$$

Definition 6.4 (recessive $x$ ). Let us say that $x$ is recessive for $\Gamma^{*}$ if for some $\delta>0$ and $\xi \in \mathcal{X}_{o}, \Gamma^{*}(x+t \xi)-$ $\Gamma^{*}(x)=t \widetilde{\Gamma^{*}}(\xi)$ for all $t \in(-\delta,+\infty)$. It is said to be non-recessive otherwise.

Proposition 6.5. We have $\bar{J}=\Gamma^{*}$ and $J^{s}=\widetilde{\Gamma^{*}}$. Moreover, $J(x)=\Gamma^{*}(x)$ for all non-recessive $x \in \mathcal{X}_{o}$.

Proof. We have already noted that $\bar{J}=\Gamma^{*}$ and by [17] (Thm. 2.3), we get: $J^{s}=\iota_{\mathrm{dom} \Gamma}^{*}$ : the support function of $\operatorname{dom} \Gamma$. Therefore, it is also the recession function of $\Gamma^{*}$. Hence, we have $\Gamma^{*}=\bar{J}=J \square J^{s}=J \square \widetilde{\Gamma^{*}}$.

Comparing $\Gamma^{*}=J \square \widetilde{\Gamma^{*}}$ with the general identity $\Gamma^{*}=\Gamma^{*} \square \widetilde{\Gamma^{*}}$, we obtain that $J(x)=\Gamma^{*}(x)$, for all nonrecessive $x \in \mathcal{X}_{o}$.

Proposition 6.6. For all $x \in \operatorname{dom} \Gamma^{*}$, we have:

$$
\Gamma^{*}(x)=\Gamma^{*}\left(x^{a}\right)+\widetilde{\Gamma^{*}}\left(x^{s}\right) .
$$

Moreover, $x$ is non-recessive if and only if $x^{s}=0$. In particular, $x^{a}$ is non-recessive. 
Proof. By (2.9), we have $\bar{I}\left(\ell_{x}\right)=I\left(\ell_{x}^{a}\right)+\widetilde{I}\left(\ell_{x}^{s}\right)$ where $\widetilde{I}$ is the recession function of $I$. It follows that $\bar{J}(x)=$ $J\left(x_{a}\right)+J^{s}\left(x^{s}\right)$, since $J\left(x_{a}\right)=I\left(\ell_{x}^{a}\right)$ (Prop. 6.3) and the recession function of $\bar{J}$ is $J^{s}$. To show this, note that $($ see $[27])$

- $I^{s}$ is the recession function of $\bar{I}$,

- the epigraph of $x \mapsto \inf \{f(\ell) ; \ell, T \ell=x\}$ (with $T$ a linear operator) is "essentially" a linear projection of the epigraph of $f$, (let us call it an inf-projection),

- the epigraph of the recession function is the recession cone of the epigraph and

- the inf-projection of a recession cone is the recession cone of the inf-projection.

The first result now follows from $\bar{J}=\Gamma^{*}$. The same set of arguments also yields the second statement.

Theorem 6.7. Let us assume that the hypotheses of Theorem 2.8 hold and that $C \cap$ icordom $\Gamma^{*} \neq \emptyset$.

(a) Then, a minimizer $\bar{x}$ of $\Gamma^{*}$ on the set $C$ is a $\Gamma^{*}$-dominating point of $C$ if and only if $\bar{x}$ is non-recessive. This is also equivalent to the following statement: "all the solutions of the minimization problem $\left(\overline{\mathrm{P}}_{C}\right)$ are absolutely continuous with respect to $R$." In such a case the solution of $\left(\overline{\mathrm{P}}_{C}\right)$ is unique and it matches the solution of $\left(\mathrm{P}_{C}\right)$.

(b) In particular when $\Gamma^{*}$ admits a degenerate recession function, i.e. $\widetilde{\Gamma^{*}}(x)=+\infty$ for all $x \neq 0$, then the minimizer $\bar{x}$ is a $\Gamma^{*}$-dominating point of $C$.

(c) The same statements hold with $\Gamma^{*}$ replaced by $\Xi$.

Proof. This is a direct consequence of Theorem 2.8, Proposition 6.6 and Lemma 5.9.

Remark 6.8. A remark about the steepness of the log-Laplace transform. In [14] (Thm. 1), with the setting of Section 5.2 where $\mathcal{X}_{o}$ is a Banach space, Kuelbs proves a result that is slightly different from statement (b) of the above theorem. It is proved that the existence of a $\Xi$-dominating point for all convex sets $C$ with a nonempty topological interior is equivalent to some property of the Gâteaux derivative of the log-Laplace transform $y \in \mathcal{X}_{o}^{\prime} \mapsto \Lambda(y)=\log \int_{\mathcal{X}_{o}} \exp (\langle y, x\rangle) R \circ \theta^{-1}(\mathrm{~d} x)$ on the boundary of its domain. This property is an infinite dimensional analogue of the steepness of the log-Laplace transform. It turns out that it is equivalent to the following assumption: the Cramér transform $\Xi=\Lambda^{*}$ admits a degenerate recession function.

Example 6.9. Csiszár's example continued. Recall that by Lemma $5.9, \Xi(x)=\Lambda^{*}(x)=\Gamma^{*}(1, x)$ so that $\Gamma$ and $\Lambda$ play the same role. Clearly, $\operatorname{dom} \Lambda=(-\infty, 1]$ and $\Lambda^{\prime}\left(1^{-}\right)=\int_{[0, \infty)} z P_{1}(d z):=x_{*}<\infty$. It follows that for all $x \geq x_{*}, \Xi(x)-\Xi\left(x_{*}\right)=x-x_{*}$.

We deduce from this that $\left(x_{*}, \infty\right)$ is a set of recessive points. By Theorem 6.7, they cannot be dominating points. Note also that the log-Laplace transform $\Lambda$ is not steep.

Remark 6.10. A remark about Csiszár's example. For comparison, suppose that $R$ in Csiszár's example 5.10 and 6.9 is replaced by its slight modification $R(\mathrm{~d} z)=\mathrm{e}^{-z} \mathrm{~d} z$ : the exponential law with parameter 1 .

Then $\Lambda(y)=-\log (1-y)+\iota_{\{y \geq 1\}}$ and $\Xi(x)=\Lambda^{*}(x)=x-1-\log x+\iota_{\{x \leq 0\}}$. Their graphic representations are given at Figure 4 and one sees that for any $c>0, R$ admits the entropic projection $P_{y}(\mathrm{~d} z)=(1-y) \mathrm{e}^{(y-1) z} \mathrm{~d} z$ which is the exponential law with parameter $(1-y)$ with $y$ such that $1 /(1-y)=\int_{[0, \infty)} z P_{y}(\mathrm{~d} z)=c$. In this case, for any $c>0$ the generalized entropic projection is the entropic projection, $\Lambda$ is steep and $\Xi$ has no recessive points. Notice however that the constraint is critical as in example 6.9.

\section{Conditional LaWs of LARGE NumBers}

In this last section, we give a probabilistic interpretation of the singular component of the generalized $I$-projection in terms of a conditional LLN. 


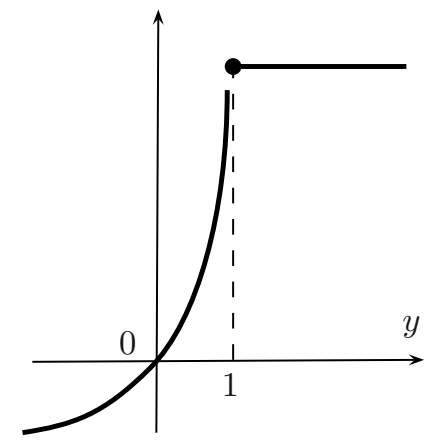

Graph of $\Lambda$

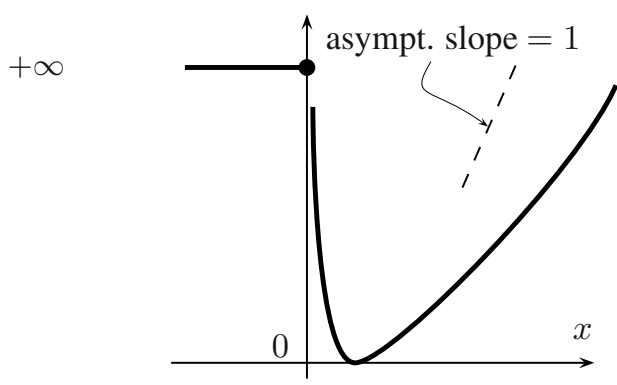

Graph of $\Xi=\Lambda^{*}$

Figure 4. The case: $R(\mathrm{~d} z)=\mathrm{e}^{-z} \mathrm{~d} z$.

\subsection{Conditional laws of large numbers}

Conditional laws of large numbers are already well-known, see [1] or [30] for instance. Theorem 7.1 below is a general statement of a conditional LLN which is inspired from [30] and [9] (Sect. 7.3). Its proof is given at Appendix B.

Let $\left\{L_{n}\right\}$ be a sequence of random vectors in the algebraic dual space $\mathcal{L}_{o}$ of some vector space $\mathcal{U}_{o}$. As a typical instance, one can think of random measures $L_{n}$ with $\mathcal{U}_{o}$ a function space. Let $T: \mathcal{L}_{o} \rightarrow \mathcal{X}_{o}$ be a linear operator with values in another vector space $\mathcal{X}_{o}$. We are going to investigate the behavior of the conditional law $\mathbb{P}\left(L_{n} \in \cdot \mid T L_{n} \in C\right)$ of $L_{n}$ as $n$ tends to infinity, for some measurable set $C$ in $\mathcal{X}_{o}$. It appears that this type of conditional law of large numbers is connected with large deviations. We assume that $\left\{L_{n}\right\}$ obeys the LDP with a good rate function $I$ in $\mathcal{L}_{o}$ endowed with the weak topology $\sigma\left(\mathcal{L}_{o}, \mathcal{U}_{o}\right)$ and the associated cylinder $\sigma$-field. It is also clear that one should assume that $\mathbb{P}\left(T L_{n} \in C\right)>0$ for all $n$, not to divide by zero. To overcome this restriction, we look at $\mathbb{P}\left(L_{n} \in \cdot \mid T L_{n} \in C_{\delta}\right)$ where $C_{\delta}$ tends to $C$ as $\delta$ tends to zero.

Let us assume that $\mathcal{X}_{o}$ is a topological vector space with its Borel $\sigma$-field and that $T: \mathcal{L}_{o} \rightarrow \mathcal{X}_{o}$ is continuous. The contraction principle tells us that

$$
X_{n}:=T L_{n}
$$

obeys the LDP in $\mathcal{X}_{o}$ with the rate function

$$
J(x)=\inf \left\{I(\ell) ; \ell \in \mathcal{L}_{o}, T \ell=x\right\} .
$$

The following set of hypotheses is based on a framework which appears in [30] and [9] (Sect. 7.3).

\subsection{Assumptions (B).}

$\left(\mathrm{B}_{L}\right)$ The sequence $\left\{L_{n}\right\}$ obeys the LDP in $\mathcal{L}_{o}$ with a good rate function $I$. This means that $I$ is inf-compact.

$\left(\mathrm{B}_{T}\right)$ The linear operator $T: \mathcal{L}_{o} \rightarrow \mathcal{X}_{o}$ is continuous.

$\left(\mathrm{B}_{C}\right)$ Assumptions on $C$. As a convention, we write $J(C)$ for $\inf _{x \in C} J(x)$.

(1) $J(C)<\infty$.

(2) The set $C$ is closed. It is the limit as $\delta$ decreases to $0: C=\cap_{\delta} C_{\delta}$, of a decreasing family of closed sets $C_{\delta}$ in $\mathcal{X}_{o}$ such that for all $\delta>0$ and all $n \geq 1, \mathbb{P}\left(X_{n} \in C_{\delta}\right)>0$

(3) and one of the following statements

(a) $C_{\delta}=C$ for all $\delta>0$ and $J($ int $C)=J(C)$ or

(b) $C \subset \operatorname{int} C_{\delta}$ for all $\delta>0$

is fulfilled.

Let $\mathcal{G}$ be the set of all solutions of the following minimization problem:

$$
\text { minimize } I(\ell) \text { subject to } T \ell \in C, \quad \ell \in \mathcal{L}_{o} \text {. }
$$


Similarly, let $\mathcal{H}$ be the set of all solutions of the following minimization problem:

$$
\text { minimize } J(x) \text { subject to } x \in C, \quad x \in \mathcal{X}_{o} \text {. }
$$

We can now state a result about conditional laws of large numbers which is proved at the Appendix.

Theorem 7.1. Assume (B). For all open subset $G$ of $\mathcal{L}_{o}$ such that $\mathcal{G} \subset G$ and all open subset $H$ of $\mathcal{X}_{o}$ such that $\mathcal{H} \subset H$, we have

$$
\begin{aligned}
& \limsup _{\delta \rightarrow 0} \limsup _{n \rightarrow \infty} \frac{1}{n} \log \mathbb{P}\left(L_{n} \notin G \mid T L_{n} \in C_{\delta}\right)<0 \text { and } \\
& \underset{\delta \rightarrow 0}{\limsup } \limsup _{n \rightarrow \infty} \frac{1}{n} \log \mathbb{P}\left(X_{n} \notin H \mid X_{n} \in C_{\delta}\right)<0 .
\end{aligned}
$$

In particular, if $C$ is convex and the rate functions $I$ and $J$ are strictly convex, we have the conditional laws of large numbers:

$$
\begin{aligned}
& \lim _{\delta} \lim _{n} \mathbb{P}\left(L_{n} \in \cdot \mid T L_{n} \in C_{\delta}\right)=\delta_{\bar{\ell}} \\
& \lim _{\delta} \lim _{n} \mathbb{P}\left(X_{n} \in \cdot \mid X_{n} \in C_{\delta}\right)=\delta_{\bar{x}}
\end{aligned}
$$

where the limits are understood with respect to the usual weak topologies of probability measures, $\bar{\ell}$ is the unique solution to the convex minimization problem (7.1) and $\bar{x}=T \bar{\ell}$ is the unique solution to (7.2).

\subsection{Empirical measures of independent samples}

Let $Z_{1}, Z_{2}, \ldots$ be an iid sequence of $\mathcal{Z}$-valued random variables which are $R$-distributed. The LDP for $L_{n}=\frac{1}{n} \sum_{i=1}^{n} \delta_{Z_{i}}$ is described by Sanov's theorem: $L_{n}$ obeys the LDP in $P_{\mathcal{Z}}$ endowed with the topology $\sigma\left(P_{\mathcal{Z}}, B_{\mathcal{Z}}\right)$ with the relative entropy $I(. \mid R)$ as its rate function, see [9] (Thm. 6.2.10). Here, $B_{\mathcal{Z}}$ denotes the space of all bounded measurable functions on $\mathcal{Z}$.

One can improve this result: this LDP still holds in $P_{\exp } \subset P_{\mathcal{Z}}$ with the topology $\sigma\left(P_{\exp }, \mathcal{E}_{\exp }\right)$ where $\mathcal{E}_{\exp }=\left\{u ; \forall a>0, \int_{\mathcal{Z}} \mathrm{e}^{a|u|} \mathrm{d} R<\infty\right\}$ is $E_{\tau}(R)$ without identifying the $R$-a.e. equal functions and $P_{\exp }=\{P \in$ $\left.P_{\mathcal{Z}} ; \int_{\mathcal{Z}}|u| \mathrm{d} P<\infty, \forall u \in \mathcal{E}_{\text {exp }}\right\}$, see [20,28].

A further improvement is obtained in [20]: $L_{n}$ obeys the LDP in $O_{\exp }$ endowed with the topology $\sigma\left(O_{\exp }, \mathcal{L}_{\exp }\right)$ with the extended relative entropy $\bar{I}(. \mid R)$ as its rate function. Here, $\mathcal{L}_{\exp }=\left\{u ; \exists a>0, \int_{\mathcal{Z}} \mathrm{e}^{a|u|} \mathrm{d} R<\infty\right\}$ is $L_{\tau}(R)$ without identifying the $R$-a.e. equal functions.

Hence, under the assumptions $\left(\mathrm{B}_{C}\right)$ on $\left\{C_{\delta}\right\}$ and $C$, we can apply Theorem 7.1 with $I=\bar{I}(. \mid R)$ and $J=\Xi$ which are defined at (3.3) and (5.3). More precisely, if the hypotheses of Proposition 5.7 are fulfilled, we see that

$$
\lim _{\delta} \lim _{n} \mathbb{P}\left(\frac{1}{n} \sum_{i=1}^{n} \delta_{Z_{i}} \in \cdot \mid \frac{1}{n} \sum_{i=1}^{n} \theta\left(Z_{i}\right) \in C_{\delta}\right)=\delta_{\widehat{P}}
$$

where $\widehat{P}$ is characterized by (5.5) and (5.7) and this limit corresponds to the usual weak topology on the set of probability measures on $P_{\exp }$ furnished with the topology $\sigma\left(P_{\exp }, \mathcal{E}_{\exp }\right)$. If the hypotheses of Proposition 5.8 are fulfilled and one of the following assumptions

(1) $\mathcal{X}_{o}=\mathbb{R}^{K}$ and $\mathcal{C} \cap$ icordom $I \neq \emptyset$ or

(2) $\|\cdot\|_{\tau^{*}-\operatorname{int}}(\mathcal{C}) \neq \emptyset$

holds, Theorem 7.1 and Proposition 5.8 tell us that (7.3) still holds, more precisely

$$
\lim _{\delta} \lim _{n} \mathbb{P}\left(\frac{1}{n} \sum_{i=1}^{n} \delta_{Z_{i}} \in \cdot \mid \frac{1}{n} \sum_{i=1}^{n} \theta\left(Z_{i}\right) \in C_{\delta}\right)=\delta_{P_{\diamond}}
$$


with respect to the same topology $\sigma\left(P_{\exp }, \mathcal{E}_{\exp }\right)$, where $P_{\diamond}$ is characterized at (5.9). See [5] for similar results w.r.t. $\sigma\left(P_{\mathcal{Z}}, B_{\mathcal{Z}}\right)$.

\subsection{A probabilistic interpretation of the singular component}

On the other hand, considering $L_{n} \in P_{\mathcal{Z}} \subset O_{\exp }$, (7.3) fails in $O_{\exp }$ endowed with topology $\sigma\left(O_{\exp }, \mathcal{L}_{\text {exp }}\right)$. Instead of this, the laws $\mathbb{P}\left(L_{n} \in \cdot \mid \frac{1}{n} \sum_{i=1}^{n} \theta\left(Z_{i}\right) \in C_{\delta}\right)$ admit cluster points which are probability measures on $O_{\exp }$ whose support consists of solutions $\hat{\ell}$ to the minimization problem (5.2). We know that $\hat{\ell}^{a}=P_{\diamond}$ for any $\hat{\ell}$.

By Proposition 2.3, $\left\langle\hat{\ell}^{s}, u\right\rangle=0$ for all $u \in \mathcal{E}_{\text {exp }}$. In particular, the mass of $\hat{\ell}^{s}$ is $\left\langle\hat{\ell}^{s}, \mathbf{1}\right\rangle=0$. Nevertheless, $\hat{\ell}^{s}$ may be nonzero. This reflects the fact for some indices $1 \leq i \leq k_{n}$ (we choose the first ones without loss of generality) with a vanishing ratio: $\lim _{n \rightarrow \infty} k_{n} / n=0$, and for some functions $u$ in $\mathcal{L}_{\exp } \backslash \mathcal{E}_{\exp }, \frac{1}{n} \sum_{i=1}^{k_{n}} u\left(Z_{i}\right)$ does not vanish as $n$ tends to infinity. Meanwhile, the remaining variables are such that $\lim _{n \rightarrow \infty} \frac{1}{n-k_{n}} \sum_{i=k_{n}+1}^{n} \delta_{Z_{i}}=P_{\diamond}$ with respect to $\sigma\left(O_{\exp }, \mathcal{L}_{\exp }\right)$.

Example 7.2. Consider example 5.10 with $c>x_{*}$. Suppose that $L_{n}-\frac{1}{n} \delta_{Z_{1}}$ is close to $P_{\diamond}=P_{1}$ and $Z_{1} / n \geq$ $c-x_{*}$ so that the event $\frac{1}{n} \sum_{i=1}^{n} Z_{i} \geq c-1 / n$ is realized. When $n$ is large, this happens with a probability $p_{n} \simeq \exp \left[-(n-1) I\left(P_{\diamond} \mid R\right)\right] \mathbb{P}\left(Z_{1} \geq n\left(c-x_{*}\right)\right)$ and with the notation of example $6.9, \lim _{n \rightarrow \infty}-\frac{1}{n} \log p_{n}=$ $I\left(P_{\diamond} \mid R\right)+\left(c-x_{*}\right)=\Gamma^{*}\left(x_{*}\right)+\left[\Gamma^{*}(c)-\Gamma^{*}\left(x_{*}\right)\right]=\Gamma^{*}(c)$. Therefore, $p_{n}$ has the optimal logarithmic behavior.

What is performed by the first particle $i=1$, may also be performed by any collection of $k_{n}$ different particles with a probability $p_{n}\left(a_{1}^{n}, \ldots, a_{k_{n}}^{n}\right) \simeq\left(\begin{array}{c}n \\ k_{n}\end{array}\right) \exp \left[-\left(n-k_{n}\right) I\left(P_{\diamond} \mid R\right)\right] \mathbb{P}\left(Z_{1} \geq a_{1}^{n}\right) \cdots \mathbb{P}\left(Z_{k_{n}} \geq a_{k_{n}}^{n}\right)$ for any $a_{1}^{n}, \ldots, a_{k_{n}}^{n}$ such that

$$
\begin{cases}\lim _{n \rightarrow \infty} k_{n} / n & =0, \\ \lim _{n \rightarrow \infty} a_{k}^{n} & =+\infty, \quad \forall 1 \leq k \leq k_{n}, \\ \lim _{n \rightarrow \infty}\left(a_{1}^{n}+\cdots+a_{k_{n}}^{n}\right) / n & =c-x_{*} .\end{cases}
$$

Again, $p_{n}\left(a_{1}^{n}, \ldots, a_{k_{n}}^{n}\right)$ is logarithmically optimal since $\lim _{n \rightarrow \infty}-\frac{1}{n} \log p_{n}\left(a_{1}^{n}, \ldots, a_{k_{n}}^{n}\right)=I\left(P_{\diamond} \mid R\right)+\lim _{n \rightarrow \infty}\left(a_{1}^{n}+\right.$ $\left.\cdots+a_{k_{n}}^{n}\right) / n=I\left(P_{\diamond} \mid R\right)+\left(c-x_{*}\right)=\Gamma^{*}(c)$.

A vanishing ratio of particles performing very large jumps is responsible for the appearance of the singular component.

For other examples of minimization problems (5.2) whose solutions exhibit a nonzero singular component, one can have a look at [19] (Sect. 7.4).

\subsection{Empirical measures with random weights}

In $[8,12]$, one gives a Bayesian interpretation of the conditional LLN of the empirical measures with random weights $L_{n}=\frac{1}{n} \sum_{i=1}^{n} W_{i} \delta_{z_{i}}$, see (1.4). Let the weights $W$ be iid random variables with a common Cramér transform $\gamma^{*}$. Let $\mathcal{Z}$ be a topological space with its Borel $\sigma$-field. Denote $C_{\mathcal{Z}}$ the space of all bounded continuous functions on $\mathcal{Z}$. The LDP for $L_{n}$ is given at [9] (Thm. 7.2.3): if $\gamma^{*}$ satisfies (4.4), $L_{n}$ obeys the LDP in $M_{\mathcal{Z}}$ with the topology $\sigma\left(M_{\mathcal{Z}}, C_{\mathcal{Z}}\right)$ and the rate function $I=I_{\gamma^{*}}$.

Under the hypotheses of Theorems 5.6 and 7.1, we obtain

$$
\lim _{\delta} \lim _{n} \mathbb{P}\left(\frac{1}{n} \sum_{i=1}^{n} W_{i} \delta_{z_{i}} \in \cdot \mid \frac{1}{n} \sum_{i=1}^{n} W_{i} \theta\left(z_{i}\right) \in C_{\delta}\right)=\delta_{Q_{\diamond}}
$$

where $Q_{\diamond}$ is described at (4.1) and the topology on $M_{\mathcal{Z}}$ is $\sigma\left(M_{\mathcal{Z}}, C_{\mathcal{Z}}\right)$.

Under the more restrictive hypotheses of Proposition 5.4, we have $Q_{\diamond}=\widehat{Q}$ which is the solution of $\left(\mathrm{P}_{C}\right)$. If the weights $W$ are nonnegative and $\mathbb{E} W=1$, the reference probability measure $R$ is interpreted as the prior distribution while $\widehat{Q}$ is a posterior distribution which is selected in $\mathcal{C}$ by means of $\left(\mathrm{P}_{C}\right)$. Hence, it is possible to estimate $\widehat{Q}$ with $L_{n}$. It is the aim of the method of Maximum Entropy in the Mean (MEM) to provide an effective simulation procedure of the posterior distribution. 
The reverse entropy corresponds to a $\gamma^{*}$ which doesn't satisfy (4.4); we have $L_{\lambda_{\diamond}}=L_{\infty}$ and $L_{\lambda_{\diamond}}^{\prime}=L_{1} R \oplus L_{\infty}^{\prime}$. This situation is investigated in details in [12] with a compact space $\mathcal{Z}$. This compactness allows to restrict the attention to the subspace $M_{\mathcal{Z}} \subset L_{1} R \oplus L_{\infty}^{\prime}$ which is decomposed into the direct sum of the spaces of measures which are absolutely continuous and singular with respect to $R$. It is proved that the $(\delta, n)$-sequence $\mathbb{P}\left(\frac{1}{n} \sum_{i=1}^{n} W_{i} \delta_{z_{i}} \in \cdot \mid \frac{1}{n} \sum_{i=1}^{n} W_{i} \theta\left(z_{i}\right) \in C_{\delta}\right)$ admits cluster points in $M_{\mathcal{Z}}$ whose support consist of solutions of $\left(\overline{\mathrm{P}}_{C}\right)$ which may have a singular component. The probabilistic interpretation of the Dirac part of this singular component is: a non-vanishing ratio of indices $i$ accumulate in areas where the $z_{i}$ are close to each other, with large values of $W_{i}$.

One can expect that $L_{n}$ obeys LDPs with respect to topologies weakened by unbounded functions, but no results of this kind appear in the literature. If this holds true, for any $\gamma^{*}$ satisfying (4.4) but such that $\lambda_{\diamond}$ isn't $\Delta_{2}$-regular, an interpretation of the singular component in terms a vanishing ratio of indices is still available, as in Section 7.4.

\section{Appendix A. Complements for the statements of Theorems 2.7 And 2.8}

The decomposition into positive and negative parts of linear forms is necessary to state the extended dual problem which is needed for the characterization of the minimizers. If $\lambda$ is not an even function, one has to consider

$$
\left\{\begin{array}{l}
\lambda_{+}(z, s)=\lambda(z,|s|) \\
\lambda_{-}(z, s)=\lambda(z,-|s|)
\end{array}\right.
$$

which are Young functions and the corresponding Orlicz spaces $L_{\lambda_{ \pm}}$.

\section{A.1. Some definitions needed for stating Theorem 2.7}

The cone $K_{\lambda}$. It is the cone of all measurable functions $u$ with a positive part $u_{+}$in $L_{\lambda_{+}}$and a negative part $u_{-}$in $L_{\lambda_{-}}: K_{\lambda}=\left\{u\right.$ measurable; $\left.\exists a>0, \int_{\mathcal{Z}} \lambda(a u) \mathrm{d} R<\infty\right\}$.

The cone $\tilde{\mathcal{Y}}$. The $\sigma\left(K_{\lambda}, L_{ \pm}\right)$-closure $\bar{A}$ of a set $A \subset K_{\lambda}$ is defined as follows: $u \in K_{\lambda}$ is in $\bar{A}$ if $u_{ \pm}$is in the $\sigma\left(L_{\lambda_{ \pm}}, L_{\lambda_{ \pm}^{*}}\right)$-closure of $A_{ \pm}=\left\{u_{ \pm} ; u \in A\right\}$. Clearly, $\bar{A}_{ \pm}=\left\{u_{ \pm} ; u \in \bar{A}\right\}$. The cone $\widetilde{\mathcal{Y}} \subset \mathcal{X}^{*}$ is the extension of $\mathcal{Y}_{o}$ which is isomorphic to the $\sigma\left(K_{\lambda}, L_{ \pm}\right)$-closure $\widetilde{T^{*} \mathcal{Y}_{o}}$ of $T^{*} \mathcal{Y}_{o}$ in $K_{\lambda}$ in the sense that $T^{*} \widetilde{\mathcal{Y}}=\widetilde{T^{*} \mathcal{Y}_{o}}$.

The extended dual problem $\left(\widetilde{\mathrm{D}}_{C}\right)$. The extended dual problem associated with $\left(\mathrm{P}_{C}\right)$ is

$$
\operatorname{maximize} \inf _{x \in C \cap \mathcal{X}}\langle\omega, x\rangle-I_{\gamma}(\langle\omega, \theta\rangle), \quad \omega \in \widetilde{\mathcal{Y}} .
$$

Note that the dual bracket $\langle\omega, x\rangle$ is meaningful for each $\omega \in \tilde{\mathcal{Y}}$ and $x \in \mathcal{X}$.

\section{A.2. Some definitions needed for stating Theorem 2.8}

Again, one needs to introduce several cones to state the extended dual problem $\overline{\mathrm{D}}_{C}$.

Recall that there is a natural order on the algebraic dual space $E^{*}$ of a Riesz vector space $E$ which is defined by: $e^{*} \leq f^{*} \Leftrightarrow\left\langle e^{*}, e\right\rangle \leq\left\langle f^{*}, e\right\rangle$ for any $e \in E$ with $e \geq 0$. A linear form $e^{*} \in E^{*}$ is said to be relatively bounded if for any $f \in E, f \geq 0$, we have $\sup _{e:|e| \leq f}\left|\left\langle e^{*}, e\right\rangle\right|<+\infty$. Although $E^{*}$ may not be a Riesz space in general, the vector space $E^{b}$ of all the relatively bounded linear forms on $E$ is always a Riesz space. In particular, the elements of $E^{b}$ admit a decomposition in positive and negative parts $e^{*}=e_{+}^{*}-e_{-}^{*}$.

The cone $K_{\lambda}^{\prime \prime}$. It is the cone of all relatively bounded linear forms $\zeta \in L_{\lambda_{\diamond}}^{\prime b}$ on $L_{\lambda_{\diamond}}^{\prime}$ with a positive part $\zeta_{+}$whose restriction to $L_{\lambda_{+}}^{\prime} \subset L_{\lambda_{\diamond}}^{\prime}$ is in $L_{\lambda_{+}}^{\prime \prime}$ and with a negative part $\zeta_{-}$whose restriction to $L_{\lambda_{-}}^{\prime} \subset L_{\lambda_{\diamond}}^{\prime}$ is in $L_{\lambda_{-}}^{\prime \prime}$ : $K_{\lambda}^{\prime \prime}=\left\{\zeta \in L_{\lambda_{\diamond}}^{\prime b} ; \zeta_{ \pm \mid L_{\lambda_{ \pm}}^{\prime}} \in L_{\lambda_{ \pm}}^{\prime \prime}\right\}$. Note that $L_{\lambda_{ \pm}}^{\prime} \subset L_{\lambda_{\diamond}}^{\prime}$.

A decomposition in $K_{\lambda}^{\prime \prime}$. Let $\rho$ be any Young function. Translating decomposition (2.17) onto $K_{\lambda}^{\prime \prime}$ leads to $K_{\lambda}^{\prime \prime}=$ $\left[K_{\lambda} \oplus K_{\lambda^{*}}^{s}\right] \oplus K_{\lambda}^{s \prime}$ where one defines $K_{\lambda^{*}}^{s}=\left\{\zeta \in\left(L_{\lambda_{\diamond}^{*}} R\right)^{b} ; \zeta_{ \pm \mid L_{\lambda_{ \pm}^{*}}} R \in L_{\lambda_{ \pm}^{*}}^{s}\right\}$ and $K_{\lambda}^{s \prime}=\left\{\zeta \in L_{\lambda_{\diamond}}^{s b} ; \zeta_{ \pm} \mid L_{\lambda_{ \pm}}^{s} \in L_{\lambda_{ \pm}}^{s \prime}\right\}$. 
Note that $L_{\lambda_{ \pm}^{*}} R \subset L_{\lambda_{\diamond}^{*}} R$ and $L_{\lambda_{ \pm}}^{s} \subset L_{\lambda_{\diamond}}^{s}$. With these cones in hand, the decomposition (2.17) holds for any $\zeta \in K_{\lambda}^{\prime \prime}$ with

$$
\left\{\begin{array}{l}
\zeta_{1}=\zeta_{1}^{a}+\zeta_{1}^{s} \in K_{\lambda} \oplus K_{\lambda^{*}}^{s}=K_{\lambda^{*}}^{\prime} \\
\zeta_{2} \in K_{\lambda}^{s \prime}
\end{array}\right.
$$

The set $\overline{\mathcal{Y}}$. The $\sigma\left(K_{\lambda}^{\prime \prime}, L_{ \pm}^{\prime}\right)$-closure $\bar{A}$ of a set $A \subset K_{\lambda}^{\prime \prime}$ is defined as follows: $\zeta \in K_{\lambda}^{\prime \prime}$ is in $\bar{A}$ if $\zeta_{ \pm}$is in the $\sigma\left(L_{\lambda_{ \pm}}^{\prime \prime}, L_{\lambda_{ \pm}}^{\prime}\right)$-closure of $A_{ \pm}=\left\{\zeta_{ \pm} ; \zeta \in A\right\}$. Clearly, $\bar{A}_{ \pm}=\left\{\zeta_{ \pm} ; \zeta \in \bar{A}\right\}$. Let $\overline{T^{*} \mathcal{Y}_{o}}$ denote the $\sigma\left(K_{\lambda}^{\prime \prime}, L_{ \pm}^{\prime}\right)$-closure of $T^{*} \mathcal{Y}_{o}$ in $K_{\lambda}^{\prime \prime}$.

Let $D$ denote the $\sigma\left(K_{\lambda}^{s \prime}, L_{ \pm}^{s}\right)$-closure of $\operatorname{dom} I_{\lambda}$, that is $\zeta \in K_{\lambda}^{s \prime}$ is in $D$ if and only if $\zeta_{ \pm}$is in the $\sigma\left(L_{\lambda_{ \pm}}^{s \prime}, L_{\lambda_{ \pm}}^{s}\right)$ closure of $\left\{u_{ \pm} ; u \in \operatorname{dom} I_{\lambda}\right\}$.

The set $\overline{\mathcal{Y}} \subset \mathcal{X}^{*}$ is the extension of $\mathcal{Y}_{o}$ which is isomorphic to $\overline{T^{*} \mathcal{Y}_{o}} \cap\left\{\zeta \in K_{\lambda}^{\prime \prime} ; \zeta_{1}^{s}=0, \zeta_{2} \in D\right\}$ in the sense that

$$
T^{*} \overline{\mathcal{Y}}=\overline{T^{*} \mathcal{Y}_{o}} \cap\left\{\zeta \in K_{\lambda}^{\prime \prime} ; \zeta_{1}^{s}=0, \zeta_{2} \in D\right\}
$$

The extended dual problem $\left(\overline{\mathrm{D}}_{C}\right)$. The extended dual problem associated with $\left(\overline{\mathrm{P}}_{C}\right)$ is

$$
\operatorname{maximize} \inf _{x \in C \cap \mathcal{X}}\langle\omega, x\rangle-I_{\gamma}\left(\left[T^{*} \omega\right]_{1}^{a}\right), \quad \omega \in \overline{\mathcal{Y}}
$$

The exact statement of Theorem 2.8(c) is

Theorem A.1. Under the assumptions of Theorem 2.8, we have

(c) Let us denote $\hat{x}:=T \hat{\ell}$. There exists $\bar{\omega} \in \overline{\mathcal{Y}}$ such that

$$
\begin{cases}(a) & \hat{x} \in C \cap \operatorname{dom} \Gamma^{*} \\ (b) & \langle\bar{\omega}, \hat{x}\rangle_{\mathcal{X}^{*}, \mathcal{X}} \leq\langle\bar{\omega}, x\rangle_{\mathcal{X}^{*}, \mathcal{X}}, \forall x \in C \cap \operatorname{dom} \Gamma^{*} \\ (c) & \hat{\ell} \in \gamma_{z}^{\prime}\left(\left[T^{*} \bar{\omega}\right]_{1}^{a}\right) R+D^{\perp}\left(\left[T^{*} \bar{\omega}\right]_{2}\right)\end{cases}
$$

where

$$
D^{\perp}(\eta)=\left\{k \in L_{\lambda_{\diamond}}^{s} ; \forall h \in L_{\lambda_{\diamond}}, \eta+h \in D \Rightarrow\langle h, k\rangle \leq 0\right\}
$$

is the outer normal cone of $D$ at $\eta$.

$T^{*} \bar{\omega}$ is in the $\sigma\left(K_{\lambda}^{\prime \prime}, L_{ \pm}^{\prime}\right)$-closure of $T^{*}\left(\left\{y \in \mathcal{Y}_{o} ; \int_{\mathcal{Z}} \lambda(\langle y, \theta\rangle) \mathrm{d} R<\infty\right\}\right)$ and there exists some $\tilde{\omega} \in \mathcal{X}_{o}^{*}$ such that

$$
\left[T^{*} \bar{\omega}\right]_{1}^{a}=\langle\tilde{\omega}, \theta(\cdot)\rangle_{\mathcal{X}_{o}^{*}, \mathcal{X}_{o}}
$$

is a measurable function in the strong closure of $T^{*}\left(\left\{y \in \mathcal{Y}_{o} ; \int_{\mathcal{Z}} \lambda(\langle y, \theta\rangle) \mathrm{d} R<\infty\right\}\right)$ in $K_{\lambda}$ : the set of all $u \in K_{\lambda}$ such that $u_{ \pm}$is in the strong closure of $T^{*}\left(\left\{y \in \mathcal{Y}_{o} ; \int_{\mathcal{Z}} \lambda(\langle y, \theta\rangle) \mathrm{d} R<\infty\right\}\right)_{ \pm}$in $L_{\lambda_{ \pm}}$.

Furthermore, $\hat{\ell} \in L_{\lambda_{\diamond}}^{\prime}$ and $\bar{\omega} \in \overline{\mathcal{Y}}$ satisfy (A.1) if and only if $\hat{\ell}$ solves $\left(\overline{\mathrm{P}}_{C}\right)$ and $\bar{\omega}$ solves $\left(\overline{\mathrm{D}}_{C}\right)$.

The dual bracket $\left\langle\left[T^{*} \bar{\omega}\right]_{2}, \hat{\ell}^{s}\right\rangle$ in Theorem 2.8(d.3) is intended to be $\left\langle\left[T^{*} \bar{\omega}\right]_{2}, \hat{\ell}^{s}\right\rangle_{K_{\lambda}^{s \prime}, K_{\lambda}^{s}}$.

\section{A.3. A complement to Theorem 4.1}

As an easy corollary of Theorem A.1 and the proof of Theorem 4.1 we obtain the following

Proposition A.2 (complement to Thm. 4.1). For each $\omega_{\diamond}$ satisfying (4.1) at Theorem 4.1, $\left\langle\omega_{\diamond}, \theta(\cdot)\right\rangle$ is in the strong closure of $T^{*} \operatorname{dom} \Gamma$ in $K_{\lambda}$ : the set of all $u \in K_{\lambda}$ such that $u_{ \pm}$is in the $\|\cdot\|_{\lambda_{ \pm}}$-closure of $\left\{\langle y, \theta\rangle_{ \pm} ; y \in\right.$ $\operatorname{dom} \Gamma\}$ in $L_{\lambda_{ \pm}}$, and there exists $\bar{\omega} \in \overline{\mathcal{Y}}$ solution of $\left(\overline{\mathrm{D}}_{C}\right)$ such that $\left[T^{*} \bar{\omega}\right]_{1}^{a}=\left\langle\omega_{\diamond}, \theta(\cdot)\right\rangle$.

Recall that $\Gamma$ is defined at (2.12). 


\section{Appendix B. Proof of Theorem 7.1}

This proof is an easy variation on [9] (Sect. 7.3). It is given for the reader's convenience. Theorem 7.1 is a restatement of Propositions B.1, B.3, B.4 and B.6 below.

We begin with the proof for $X_{n}$. As $T$ is continuous and $I$ is a good rate function, $J$ is also a good rate function. Let us first state the upper bound of a conditional LDP.

Proposition B.1. Under the assumptions of Theorem \%.1, for all closed subset $F$ of $\mathcal{X}_{o}$, we have

$$
\limsup _{\delta \rightarrow 0} \limsup _{n \rightarrow \infty} \frac{1}{n} \log \mathbb{P}\left(X_{n} \in F \mid X_{n} \in C_{\delta}\right) \leq-J_{C}(F)
$$

where

$$
J_{C}(x):= \begin{cases}J(x)-J(C) & \text { if } x \in C \\ +\infty & \text { if } x \notin C\end{cases}
$$

Proof. Clearly, for all measurable set $B$ and all $n \geq 1, \delta>0$, we have $\frac{1}{n} \log \mathbb{P}\left(X_{n} \in B \mid X_{n} \in C_{\delta}\right)=\frac{1}{n} \log \mathbb{P}\left(X_{n} \in\right.$ $\left.B \cap C_{\delta}\right)-\frac{1}{n} \log \mathbb{P}\left(X_{n} \in C_{\delta}\right)$. Hence,

$$
\begin{aligned}
\limsup _{n} \frac{1}{n} \log \mathbb{P}\left(X_{n} \in F \mid X_{n} \in C_{\delta}\right) & \leq \limsup _{n} \frac{1}{n} \log \mathbb{P}\left(X_{n} \in F \cap \operatorname{cl} C_{\delta}\right)-\liminf _{n} \frac{1}{n} \log \mathbb{P}\left(X_{n} \in \operatorname{int} C_{\delta}\right) \\
& \leq-J\left(F \cap C_{\delta}\right)+J\left(\operatorname{int} C_{\delta}\right) \\
& \leq-J\left(F \cap C_{\delta}\right)+J(C)
\end{aligned}
$$

where the last inequality follows from the assumption $\left(\mathrm{B}_{C}^{3}\right)$. We complete the proof with the following lemma.

Lemma B.2. For any closed set $F, \lim _{\delta} J\left(F \cap C_{\delta}\right)=J(F \cap C) \in[0, \infty]$.

Proof. As $C \subset C_{\delta}$, for all $\delta>0$, we have $J\left(F \cap C_{\delta}\right) \leq J(F \cap C)$. Since $C_{\delta}$ is decreasing, $J\left(F \cap C_{\delta}\right)$ is nondecreasing and $\lim _{\delta} J\left(F \cap C_{\delta}\right)=\sup _{\delta} J\left(F \cap C_{\delta}\right) \in[0, \infty]$. If $\sup _{\delta} J\left(F \cap C_{\delta}\right)=\infty$, the inequality $J\left(F \cap C_{\delta}\right) \leq J(F \cap C)$ leads to the desired result.

Now, let us suppose that $\sup _{\delta} J\left(F \cap C_{\delta}\right)<\infty$. As $F \cap C_{\delta}$ is closed and $J$ is inf-compact, for any $\delta$ there exists $x_{\delta} \in F \cap C_{\delta}$ such that $J\left(x_{\delta}\right)=J\left(F \cap C_{\delta}\right)$ and we can extract a converging subsequence $x_{k} \rightarrow x_{*}$. Because the $C_{\delta}$ 's are decreasing, we get $\cap_{\delta} C_{\delta}=\cap_{k} C_{\delta_{k}}$ and $\lim _{\delta} J\left(F \cap C_{\delta}\right)=\lim _{k} J\left(x_{k}\right)$. More, $x_{*} \in F \cap\left(\cap_{k} C_{\delta_{k}}\right)=F \cap C$ and as $J$ is lsc: $\lim _{k} J\left(x_{k}\right) \geq J\left(x_{*}\right) \geq J(F \cap C)$. Therefore, $\lim _{\delta} J\left(F \cap C_{\delta}\right) \geq J(F \cap C)$ which completes the proof.

Let us state the lower bound corresponding to Proposition B.1.

Proposition B.3. If the assumption $\left(\mathrm{B}_{C}^{3}\right)$ on the conditioning event is restricted to (3b):

$$
C \subset \operatorname{int} C_{\delta}, \forall \delta>0
$$

then, for all open subset $G$ of $\mathcal{X}_{o}$, we have

$$
\liminf _{\delta \rightarrow 0} \liminf _{n \rightarrow \infty} \frac{1}{n} \log \mathbb{P}\left(X_{n} \in G \mid X_{n} \in C_{\delta}\right) \geq-J_{C}(G) .
$$

Proof. For all $\delta>0$,

$$
\begin{aligned}
\liminf _{n} \frac{1}{n} \log \mathbb{P}\left(X_{n} \in G \mid X_{n} \in C_{\delta}\right) & \geq \liminf _{n} \frac{1}{n} \log \mathbb{P}\left(X_{n} \in G \cap \operatorname{int} C_{\delta}\right)-\limsup _{n} \frac{1}{n} \log \mathbb{P}\left(X_{n} \in C_{\delta}\right) \\
& \geq-J\left(G \cap \operatorname{int} C_{\delta}\right)+J\left(C_{\delta}\right) \\
& \geq-J(G \cap C)+J\left(C_{\delta}\right) .
\end{aligned}
$$


We conclude with Lemma B.2.

Let us recall that $\mathcal{H}=\operatorname{argmin} J_{C}$ is the set of the minimizers of $J$ on $C$. As $C$ is closed and $J$ is inf-compact, $\mathcal{H}$ is a compact set. As an immediate corollary of Proposition B.1, we have the following conditional LLN which is the part of the statement of Theorem 7.1 concerning $X_{n}$.

Proposition B.4. For all open subset $H$ of $\mathcal{X}_{o}$ such that $\mathcal{H} \subset H$, we have

$$
\limsup _{\delta \rightarrow 0} \limsup _{n \rightarrow \infty} \frac{1}{n} \log \mathbb{P}\left(X_{n} \notin H \mid X_{n} \in C_{\delta}\right)<0 .
$$

Let us now have a look at $L_{n}$. We are interested in the asymptotic behavior of $\mathbb{P}\left(L_{n} \in \cdot \mid T L_{n} \in C_{\delta}\right)$ with $C_{\delta} \subset \mathcal{X}_{o}$. Let us denote $A_{\delta}:=T^{-1}\left(C_{\delta}\right)=\left\{\ell \in \mathcal{L}_{o} ; T \ell \in C_{\delta}\right\}$ and $A=T^{-1} C$. It is useful to state the assumptions on the $C_{\delta}$ 's rather than on the $A_{\delta}$ 's. In fact, we have the following transfer result.

Lemma B.5. We assume that $T$ is continuous.

(a) If $C$ is closed and $J(C)=J(\operatorname{int} C)$, then $A$ is closed and $I(A)=I(\operatorname{int} A)$.

(b) If $C:=\cap_{\delta} C_{\delta} \subset \operatorname{int} C_{\delta}$ for all $\delta>0$, then $A=\cap_{\delta} \mathrm{cl} A_{\delta}$ and $A \subset \operatorname{int} A_{\delta}$ for all $\delta>0$.

Proof. Since $T$ is continuous, for any $A^{\prime}=T^{-1} C^{\prime}$, we have: $T^{-1}\left(\operatorname{int} C^{\prime}\right) \subset \operatorname{int} A^{\prime} \subset A^{\prime} \subset \operatorname{cl} A^{\prime} \subset T^{-1}\left(\operatorname{cl} C^{\prime}\right)$.

Let us begin with (a). As $C$ is closed, so is $A$. For any $A=T^{-1} C$, we have $I(A)=\inf \left\{\Phi^{*}(\ell) ; T \ell \in\right.$ $C\}=\inf _{x \in C} \inf \left\{\Phi^{*}(\ell) ; T \ell=x\right\}=\inf _{x \in C} J(x)=J(C)$. Hence, $I(A)=J(C)=J(\operatorname{int} C)$ (by hypothesis) $=$ $I\left(T^{-1}(\operatorname{int} C) \geq I(\operatorname{int} A)\right.$, since $T^{-1}(\operatorname{int} C) \subset \operatorname{int} A$. But the converse inequality: $I(A) \leq I(\operatorname{int} A)$ is clear.

Let us prove (b). We have: $\cap_{\delta} \operatorname{cl} A_{\delta} \subset T^{-1}\left(\cap_{\delta} C_{\delta}\right):=A:=T^{-1}(C) \subset T^{-1}\left(\cap_{\delta} \operatorname{int} C_{\delta}\right)$ (by hypothesis) $\subset \cap_{\delta} \operatorname{int} A_{\delta} \subset \cap_{\delta} \mathrm{cl} A_{\delta}$. This proves that all these sets are equal, and in particular that $A=\cap_{\delta} \operatorname{cl} A_{\delta}$. On the other hand, as for any $\delta>0, C \subset \operatorname{int} C_{\delta}$, we have $A=T^{-1}(C) \subset T^{-1}\left(\operatorname{int} C_{\delta}\right) \subset \operatorname{int} A_{\delta}$.

Let us recall that $\mathcal{G}$ is the set of the minimizers of $I$ on $A$. By the above Lemma B.5, in the situation of the $L_{n}$ 's, Proposition B.4 becomes the following

Proposition B.6. Under our general assumptions, for all open subset $G$ of $\mathcal{L}_{o}$ such that $\mathcal{G} \subset G$, we have

$$
\limsup _{\delta \rightarrow 0} \limsup _{n \rightarrow \infty} \frac{1}{n} \log \mathbb{P}\left(L_{n} \notin G \mid T L_{n} \in C_{\delta}\right)<0 .
$$

Note that by Lemma B.5, the $A_{\delta}$ 's share the same properties as the $C_{\delta}$ 's. In particular, $I\left(\operatorname{int} A_{\delta}\right) \leq I(A)$ also holds for them.

\section{REFERENCES}

[1] R. Azencott, Grandes déviations et applications, in École d'Eté de Probabilités de Saint-Flour VIII (1978).

[2] J.M. Borwein and A.S. Lewis, Strong rotundity and optimization. SIAM J. Optim. 1 (1994) 146-158.

[3] C. Boucher, R.S. Ellis and B. Turkington, Spatializing random measures: doubly indexed processes and the large deviation principle. Ann. Probab. 27 (1999) 297-324.

[4] I. Csiszár, I-divergence geometry of probability distributions and minimization problems. Ann. Probab. 3 (1975) $146-158$.

[5] I. Csiszár, Sanov property, generalized I-projection and a conditional limit theorem. Ann. Probab. 12 (1984) 768-793.

[6] I. Csiszár, Generalized projections for non-negative functions. Acta Math. Hungar. 68 (1995) 161-185.

[7] I. Csiszár, F. Gamboa and E. Gassiat, MEM pixel correlated solutions for generalized moment and interpolation problems. IEEE Trans. Inform. Theory 45 (1999) 2253-2270.

[8] D. Dacunha-Castelle and F. Gamboa, Maximum d'entropie et problème des moments. Ann. Inst. H. Poincaré. Probab. Statist. 26 (1990) 567-596.

[9] A. Dembo and O. Zeitouni, Large Deviations Techniques and Applications. Second edition. Appl. Math. 38. Springer-Verlag (1998).

[10] U. Einmahl and J. Kuelbs, Dominating points and large deviations for random vectors. Probab. Theory Relat. Fields 105 (1996) 529-543. 
[11] R.S. Ellis, J. Gough and J.V. Puli, The large deviations principle for measures with random weights. Rev. Math. Phys. 5 (1993) 659-692.

[12] F. Gamboa and E. Gassiat, Bayesian methods and maximum entropy for ill-posed inverse problems. Ann. Statist. 25 (1997) 328-350.

[13] H. Gzyl, The Method of Maximum Entropy. World Scientific (1994).

[14] J. Kuelbs, Large deviation probabilities and dominating points for open convex sets: nonlogarithmic behavior. Ann. Probab. 28 (2000) 1259-1279.

[15] C. Léonard, Large deviations for Poisson random measures and processes with independent increments. Stoch. Proc. Appl. 85 (2000) 93-121.

[16] C. Léonard, Convex minimization problems with weak constraint qualifications. J. Convex Anal. 17 (2010) 321-348.

[17] C. Léonard, Minimization of energy functionals applied to some inverse problems. J. Appl. Math. Optim. 44 (2001) $273-297$.

[18] C. Léonard, Minimizers of energy functionals under not very integrable constraints. J. Convex Anal. 10 (2003) 63-88.

[19] C. Léonard, Minimization of entropy functionals. J. Math. Anal. Appl. 346 (2008) 183-204.

[20] C. Léonard and J. Najim, An extension of Sanov's theorem: application to the Gibbs conditioning principle. Bernoulli 8 (2002) $721-743$.

[21] J. Najim, A Cramér type theorem for weighted random variables. Electron. J. Probab. 7 (2002) 1-32.

[22] P. Ney, Dominating points and the asymptotics of large deviations for random walks on $R^{d}$. Ann. Probab. 11 (1983) $158-167$.

[23] P. Ney, Convexity and large deviations. Ann. Probab. 12 (1984) 903-906.

[24] M.M. Rao and Z.D. Ren, Theory of Orlicz spaces, Pure Appl. Math. 146. Marcel Dekker, Inc. (1991).

[25] R.T. Rockafellar, Integrals which are convex functionals. Pacific J. Math. 24 (1968) 525-539.

[26] R.T. Rockafellar, Conjugate Duality and Optimization, volume 16 of Regional Conf. Series in Applied Mathematics. SIAM, Philadelphia (1974)

[27] R.T. Rockafellar and R. Wets, Variational Analysis, in Grundlehren der Mathematischen Wissenschaften, volume 317. Springer (1998).

[28] A. Schied, Cramér's condition and Sanov's theorem. Statist. Probab. Lett. 39 (1998) 55-60.

[29] C.R. Smith, G.J. Erickson and P.O. Neudorfer (Eds.). Maximum Entropy and Bayesian Methods, Proc. of 11th Int. Workshop on Maximum Entropy and Bayesian Methods of Statistical Analysis, Seattle, 1991. Kluwer.

[30] D.W. Stroock and O. Zeitouni, Microcanonical distributions, Gibbs states and the equivalence of ensembles, in Festchrift in Honour of F. Spitzer, edited by R. Durrett and H. Kesten. Birkhaüser (1991) 399-424. 


\section{INDEX}

Assumptions (A)

$\left(\mathrm{A}_{R}\right)$ on the measure $R, 349$

$\left(\mathrm{A}_{C}\right)$ on the set $C, 349$

$\left(\mathrm{A}_{\gamma^{*}}\right)$ on $\gamma^{*}, 349$

$\left(\mathrm{A}_{\theta}\right)$ on $\theta, 350$

$\left(\mathrm{A}_{\theta}^{\forall}\right), 351$

$\left(\mathrm{A}_{\theta}^{\exists}\right), 351$

Assumptions (B)

$\left(\mathrm{B}_{L}\right)$ on $\left\{L_{n}\right\}, 372$

$\left(\mathrm{B}_{T}\right)$ on $T, 372$

$\left(\mathrm{B}_{C}\right)$ on the constraint set $C, 372$

$B_{\mathcal{Z}}$, bounded measurable functions on $\mathcal{Z}, 373$

$\mathcal{C}$, constraint set, $343,346,358$

$C$, constraint set, 346,358

$\overline{\mathcal{C}}$, extended constraint set, 358

convex conjugate, $f^{*}$, see (1.12), 347

$C_{\mathcal{Z}}$, bounded continuous functions on $\mathcal{Z}, 374$

dominating point, see Definition 6.1, 369

Dual problems

$\left(\mathrm{D}_{C}\right), 352$

$\left(\widetilde{\mathrm{D}}_{C}\right), 352,375$

$\left(\overline{\mathrm{D}}_{C}\right), 354,376$

(D), 361

$\left(\mathrm{D}_{E}\right), 363$

$\mathcal{E}_{\exp }, 373$

entropic projection, see Definition 5.2, 365

$E_{\tau}, 356$

$\Phi_{E}, \Phi_{L}, 360$

$\Phi_{E}^{*}, \Phi_{L}^{*}, 360$

Functions of $(s, z)$ or $(t, z)$

$\gamma$, see (2.4), 349

$\gamma^{*}$, integrand, see (1.5), 344, 345, 349

$\lambda$, see (2.4), 349

$\lambda_{\diamond}$, see $(2.5), 350$

$m$, see $\left(\mathrm{A}_{\gamma^{*}}\right), 349$

$\rho$, Young function, 348

$\tau, 356$

$\tau^{*}, 356$

$\mathcal{G}, 373$

$\Gamma$, see $(2.12), 352$

$\Gamma^{*}$, see $(2.13), 352$

$\widetilde{\Gamma^{*}}$, see $(6.2), 370$

generalized entropic projection, see Definition 5.2, 365

generalized $I$-projection, see Definition 5.2, 365

$\mathcal{H}, 373$

$\iota_{A}$, convex indicator, see (1.7), 345

$I_{\varphi}$, integral functional, 348

$I$, entropy, see (2.6), (4.6), 344, 350, 360

$\bar{I}$, extended entropy, see (2.9), (4.6), 351, 360

$I^{s}$, singular entropy, see (2.10), 351

$I(\cdot \mid R)$, relative entropy, see $(3.1), 344,355$
$\bar{I}(\cdot \mid R)$, extended relative entropy, see (3.3), 356 icor, 348

icordom, 348

inf-convolution, $f \square g, 348$

$I$-projection, see Definition 5.2, 365

$J, 370,372$

$\bar{J}, J^{s}, 370$

$K_{\lambda}, 375$

$K_{\lambda}^{\prime \prime}, 376$

$\Lambda$, log-Laplace transform of $R \circ \theta^{-1}$, see (5.10), 368

$\mathcal{L}_{\text {exp }}, 373$

$\hat{\ell}$, solution to $\left(\overline{\mathrm{P}}_{C}\right)$, see $(2.19), 354$

$L_{\lambda_{\diamond}}, 350$

$\mathcal{L}_{o}, 372$

$L_{\lambda_{\diamond}^{*}}, 350$

$L_{n}, 343,344,372$

$L_{\tau}, 356$

$L_{\tau^{*}}, 356$

$M_{\mathcal{Z}}$, space of signed measures on $\mathcal{Z}, 344$

non-recessive $x$, see Definition 6.4, 370

$\tilde{\omega}$, see (2.15), 353

$\bar{\omega}$, see (2.19), (A.1), 354, 376

$\omega_{\diamond}$, see $(4.1), 357,376$

$O_{\text {exp }}$, see (3.4), 356

Orlicz spaces, 348

decomposition

$\ell^{a}$, absolutely continuous part of $\ell$, see (2.2), 349

$\ell^{s}$, singular part of $\ell$, see (2.2), 349

$\zeta_{1}^{a}, \zeta_{1}^{s}, \zeta_{2}$, see (2.17), 353

$L_{\rho^{*}} R$, space of absolutely continuous forms, 349

$L_{\rho}^{s}$, space of singular forms, 349

$\Delta_{2}$-condition, see (2.3), 349

$E_{\rho}$, small Orlicz space, 348

$L_{\rho}$, Orlicz space, 348

$\|\cdot\|_{\rho}$, Luxemburg norm, 348

$\rho$, Young function, 348

$P_{\diamond}$, see (5.9), 367

$P_{\text {exp }}, 373$

$\widehat{P}$, see (5.5), 367

Primal problems

$\left(\mathrm{P}_{C}\right), 351$

$\left(\overline{\mathrm{P}}_{C}\right), 351$

$\left(\mathcal{P}_{\mathcal{C}}\right), 358$

$\left(\mathrm{P}_{E}\right), 361$

$\left(\mathrm{P}_{L}\right), 361$

$(\mathcal{P}), 361$

$P_{\mathcal{Z}}$, set of probability measures on $\mathcal{Z}, 343$

$Q_{\diamond}$, see (4.1), 357

$\widehat{Q}$, minimizer of $\left(\mathrm{P}_{C}\right), 352$

$Q_{*}$, generalized $I$-projection, 365 
$R$, reference measure, 344, 349

recessive $x$, see Definition $6.4,370$

reverse relative entropy, 345

$T$, constraint operator, see (2.8), 350

$\theta$, constraint function, see $(2.8), 346,349,350$

$T^{*}$, adjoint of $T, 352$

$\mathcal{U}_{o}, 372$

$\hat{x}$, see $(2.15),(2.19), 353,354$

$\Xi$, see $(5.3), 366$
$\Xi$-dominating point, see Definition 6.2, 369

$\mathcal{X}$, topological dual of $\mathcal{Y}_{o}$ and $\mathcal{Y}, 352$

$\mathcal{X}_{o}$, algebraic dual of $\mathcal{Y}_{o}, 349$

$\bar{x}^{a}, \bar{x}^{s}, 358$

$x^{a}, x^{s}, 370$

$\mathcal{Y}$, completion of $\mathcal{Y}_{o}, 351$

$\mathcal{Y}_{o}, 349$

$\overline{\mathcal{Y}}, 354,376$

$\widetilde{\mathcal{Y}}, 352,375$

$\mathcal{Z}$, reference space, 349 